\title{
Elaboration of Diarylketones Into Naphthalenes Fused on Two or Four Sides: A Naphthoannulation Procedure
}

\author{
Patrick M. Donovan and Lawrence T. Scott* \\ Department of Chemistry, Merkert Chemistry Center, \\ Boston College, Chestnut Hill, MA 02467-3860
}

\section{Experimental Section}

Corey-Fuchs Olefination of Benzophenone. Benzophenone (8a, $3.039 \mathrm{~g}, 16.68 \mathrm{mmol})$, carbon tetrabromide $(11.064 \mathrm{~g}, 33.361 \mathrm{mmol})$, and triphenyl phosphine $(17.530 \mathrm{~g}, 66.840 \mathrm{mmol})$ were combined in $100 \mathrm{~mL}$ of anhydrous benzene as described in General Procedure 1. The reaction was run at $145^{\circ} \mathrm{C}$ for $64 \mathrm{~h}$. Column chromatography of the crude product on silica gel with hexanes as the eluent gave $4.457 \mathrm{~g}$ (79\% yield) of 1,1-dibromo-2,2-diphenylethene as a yellow crystalline solid that was pure by ${ }^{1} \mathrm{H}$ NMR analysis. The ${ }^{1} \mathrm{H}$ NMR spectrum matched that reported in the literature. ${ }^{1}{ }^{13} \mathrm{C}$ NMR $\left(100 \mathrm{MHz} \mathrm{CDCl}_{3}\right) \delta 147.87,141.42,128.81,128.42$, $128.05,90.47$.

Sonogashira Coupling of 1,1-dibromo-2,2-diphenylethene. The 1,1-dibromo-2,2diphenylethene (1.202 g, $3.558 \mathrm{mmol}), \mathrm{PdCl}_{2}\left(\mathrm{PPh}_{3}\right)_{2}$ (147 mg, $\left.0.209 \mathrm{mmol}\right)$, copper(I) iodide (74 $\mathrm{mg}, 0.39 \mathrm{mmol})$, and trimethylsilyl acetylene $(2.25 \mathrm{~mL}, 15.9 \mathrm{mmol})$ were combined in $35 \mathrm{~mL}$ of triethylamine as described in General Procedure 2. The reaction was run at $95{ }^{\circ} \mathrm{C}$ for $65 \mathrm{~h}$. Column chromatography of the crude product on silica gel with a 5\% ethyl acetate: $95 \%$ hexanes solution as the eluent gave $1.245 \mathrm{~g}$ (94\% yield) of 1,1-diphenyl-2,2-bis(trimethylsilylethynyl)ethene as a sticky brown solid that was pure by ${ }^{1} \mathrm{H}$ NMR analysis: $\mathrm{mp} 124-126{ }^{\circ} \mathrm{C}$. ${ }^{1} \mathrm{H}$ NMR (400 MHz, $\left.\mathrm{CDCl}_{3}\right) \delta(\mathrm{ppm}): 7.43-7.41(\mathrm{~m}, 4 \mathrm{H}), 7.32-7.27(\mathrm{~m}, 6 \mathrm{H}), 0.099(\mathrm{~s}, 18 \mathrm{H}) .{ }^{13} \mathrm{C}$ NMR $\left(100 \mathrm{MHz}, \mathrm{CDCl}_{3}\right) \delta 157.90,139.71,129.99,128.14,127.17,102.95,101.60,97.59,-0.42$. HRMS ESI $(\mathrm{m} / z)$ : $[\mathrm{M}+\mathrm{Na}]^{+}$calcd for $\mathrm{C}_{24} \mathrm{H}_{28} \mathrm{NaSi}_{2}$, 395.1627. Found 395.1627.

Desilylation of 1,1-diphenyl-2,2-bis(trimethylsilylethynyl)ethene. The 1,1-diphenyl-2,2bis(trimethylsilylethynyl)ethene $(116 \mathrm{mg}, 0.312 \mathrm{mmol})$ and tetrabutylammonium fluoride trihydrate $(252 \mathrm{~g}, 0.965 \mathrm{mmol})$ were stirred in $4 \mathrm{~mL}$ of dichloromethane as described in General Procedure 3 until TLC showed no remaining starting material (ca $30 \mathrm{~min}$ ). Care was taken not to apply excessive heat during the workup. The crude 1,1-diethynyl-2,2-diphenylethene was immediately subjected to the next reaction without further purification. ${ }^{1} \mathrm{H}$ NMR $(300 \mathrm{MHz}$, $\left.\mathrm{CDCl}_{3}\right) \delta(\mathrm{ppm}):$ 7.44-7.31 (m, 10H), $3.02(\mathrm{~s}, 2 \mathrm{H})$.

Double Cyclization of 1,1-diethynyl-2,2-diphenylethene. A solution of 1,1-diethynyl-2,2diphenylethene in 1,2-dichloroethane ( $3 \mathrm{~mL}$ total volume) was added at a rate of $0.3 \mathrm{~mL} / \mathrm{h}$ with a syringe pump to a solution of $\mathrm{RuCl}_{2}\left(\mathrm{PPh}_{3}\right)\left(\eta^{6} \text {-para-cymene }\right)^{2}(56 \mathrm{mg}, 0.098 \mathrm{mmol})$ and ammonium hexafluorophosphate $(30 \mathrm{mg}, 0.18 \mathrm{mmol})$ in $27 \mathrm{~mL}$ of 1,2-dichloroethane at $75^{\circ} \mathrm{C}$ as described in General Procedure 4. Heating was continued for an additional $54 \mathrm{~h}$ after completion

1. Li, G.; Warner, P. M. Tetrahedron Lett. 1995, 36, 8573-8576.

2. Bennett, M. A. S., Anthony K. J. Chem. Soc., Dalton 1974, 2, 233-241. 
of the addition, for a total reaction time of $64 \mathrm{~h}$. Column chromatography of the crude product on silica gel with hexanes as the eluent gave $42 \mathrm{mg}$ of benzo[c]phenanthrene (9a) as an off-white powder (60\% yield over 2 steps) that was pure by ${ }^{1} \mathrm{H}$ NMR analysis. The ${ }^{1} \mathrm{H}$ NMR spectrum matched that reported in the literature. ${ }^{3}$

Corey-Fuchs Olefination of $\mathbf{4 , 4}$ '-di-t-butylbenzophenone. The 4,4'-di- $t$-butylbenzophenone $(\mathbf{8 b}, 2.547 \mathrm{~g}, 8.649 \mathrm{mmol})$, carbon tetrabromide $(6.088 \mathrm{~g}, 18.36 \mathrm{mmol})$, and triphenyl phosphine $(9.642 \mathrm{~g}, 36.76 \mathrm{mmol})$ were combined in $50 \mathrm{~mL}$ of anhydrous benzene as described in General Procedure 1. The reaction was run at $150{ }^{\circ} \mathrm{C}$ for $100 \mathrm{~h}$. Column chromatography of the crude product on silica gel with hexanes as the eluent gave $2.030 \mathrm{~g}$ (52\% yield) of 1,1-dibromo-2,2bis(4-t-butylphenyl)ethene as a yellowish solid that was pure by ${ }^{1} \mathrm{H}$ NMR analysis: $\mathrm{mp}$ 157-159 ${ }^{\circ} \mathrm{C}$. ${ }^{1} \mathrm{H} \mathrm{NMR}\left(400 \mathrm{MHz}, \mathrm{CDCl}_{3}\right) \delta(\mathrm{ppm}): 7.33(\mathrm{dt}, J=8.4 \mathrm{~Hz}$ and $J=2.0 \mathrm{~Hz}, 4 \mathrm{H}), 7.21(\mathrm{dt}, J=$ $8.8 \mathrm{~Hz}$ and $J=2.2 \mathrm{~Hz}, 4 \mathrm{H}), 1.30(\mathrm{~s}, 18 \mathrm{H}) .{ }^{13} \mathrm{C} \mathrm{NMR}\left(125 \mathrm{MHz}, \mathrm{CDCl}_{3}\right) \delta 151.01,148.06$, 138.78, 128.63, 125.34, 89.51, 34.85, 31.49. Anal. calcd for $\mathrm{C}_{22} \mathrm{H}_{26} \mathrm{Br}_{2}: \mathrm{C}, 58.69 ; \mathrm{H}, 5.82$. Found: C, 58.33; H, 5.70.

Sonogashira Coupling of 1,1-dibromo-2,2-bis(4-t-butylphenyl)ethene. The 1,1-dibromo2,2-bis(4-t-butylphenyl)ethene (1.004 g, $2.229 \mathrm{mmol}), \mathrm{PdCl}_{2}\left(\mathrm{PPh}_{3}\right)_{2}$ (256 mg, $\left.0.364 \mathrm{mmol}\right)$, copper(I) iodide $(141 \mathrm{mg}, 0.741 \mathrm{mmol})$, and trimethylsilyl acetylene $(1.60 \mathrm{~mL}, 11.3 \mathrm{mmol})$ were combined in $26 \mathrm{~mL}$ of triethylamine as described in General Procedure 2. The reaction was run at $100{ }^{\circ} \mathrm{C}$ for $20 \mathrm{~h}$. Column chromatography of the crude product on silica gel with a $1 \%$ ethyl acetate: $99 \%$ hexanes solution as the eluent gave $770 \mathrm{mg}$ (71\% yield) of 1,1-bis(4-tbutylphenyl)-2,2-bis(trimethylsilylethynyl)ethene as an orange-brown solid that was pure by ${ }^{1} \mathrm{H}$ NMR analysis: $\mathrm{mp} 171-173{ }^{\circ} \mathrm{C} .{ }^{1} \mathrm{H}$ NMR $\left(400 \mathrm{MHz}, \mathrm{CDCl}_{3}\right) \delta(\mathrm{ppm}): 7.37(\mathrm{dm}, \mathrm{J}=8.8 \mathrm{~Hz}$, 4H), $7.30(\mathrm{dm}, \mathrm{J}=8.8 \mathrm{~Hz}, 4 \mathrm{H}), 1.32(\mathrm{~s}, 18 \mathrm{H}), 0.09(\mathrm{~s}, 18 \mathrm{H}) .{ }^{13} \mathrm{C} \mathrm{NMR}\left(100 \mathrm{MHz}, \mathrm{CDCl}_{3}\right) \delta$ $158.89,151.56,137.22,130.13,124.42,103.87,100.99,97.47,35.00,31.60, .035$. HRMS ESI $(\mathrm{m} / \mathrm{z}):[\mathrm{M}+\mathrm{Na}]^{+}$calcd for $\mathrm{C}_{32} \mathrm{H}_{44} \mathrm{NaSi}_{2}$, 507.2879. Found 507.2883.

Desilylation of 1,1-bis(4-t-butylphenyl)-2,2-bis(trimethylsilylethynyl)ethene. The 1,1-bis(4$t$-butylphenyl)-2,2-bis(trimethylsilylethynyl)ethene (102 $\mathrm{mg}, 0.211 \mathrm{mmol}$ ) and tetrabutylammonium fluoride trihydrate $(245 \mathrm{mg}, 0.776 \mathrm{mmol})$ were stirred in $8 \mathrm{~mL}$ of dichloromethane as described in General Procedure 3 until TLC showed no remaining starting material (ca 45 min). Care was taken not to apply excessive heat during the workup. The crude 1,1-diethynyl-2,2-bis(4-t-butylphenyl)ethene was immediately subjected to the next reaction without further purification. ${ }^{1} \mathrm{H}$ NMR $\left(400 \mathrm{MHz}, \mathrm{CDCl}_{3}\right) \delta(\mathrm{ppm}): 7.37-7.32(\mathrm{~m}, 8 \mathrm{H}), 3.02(\mathrm{~s}, 2 \mathrm{H}), 1.32(\mathrm{~s}$, $18 \mathrm{H})$.

Double Cyclization of 1,1-diethynyl-2,2-bis(4-t-butylphenyl)ethene. A solution of 1,1diethynyl-2,2-bis(4-t-butylphenyl)ethene in 1,2-dichloroethane (4 mL total volume) was added at a rate of $0.2 \mathrm{~mL} / \mathrm{h}$ with a syringe pump to a solution of $\mathrm{RuCl}_{2}\left(\mathrm{PPh}_{3}\right)\left(\eta^{6} \text {-para-cymene }\right)^{2}(21 \mathrm{mg}$, $0.036 \mathrm{mmol})$ and ammonium hexafluorophosphate $(13 \mathrm{mg}, 0.080 \mathrm{mmol})$ in $10 \mathrm{~mL}$ of $1,2-$ dichloroethane at $85{ }^{\circ} \mathrm{C}$ as described in General Procedure 4. Heating was continued for an additional $4 \mathrm{~h}$ after completion of the addition, for a total reaction time of $24 \mathrm{~h}$. Column chromatography of the crude product on neutral alumina with a solution of $1 \%$ ethyl acetate:

3. Bax, A.; Ferretti, J. A.; Nashed, N.; Jerina, D. M. J. Org. Chem. 1985, 50, 3029-3034. 
99\% hexanes as the eluent gave $43 \mathrm{mg}$ of 3,10-di-t-butylbenzo[c]phenanthrene (9b) as an offwhite powder $\left(60 \%\right.$ yield over 2 steps) that was pure by ${ }^{1} \mathrm{H}$ NMR analysis: mp $131-133{ }^{\circ} \mathrm{C}$. ${ }^{1} \mathrm{H}$ NMR (300 MHz, $\left.\mathrm{CDCl}_{3}\right) \delta(\mathrm{ppm}): 9.09(\mathrm{~d}, J=9.0 \mathrm{~Hz}, 2 \mathrm{H}), 7.95(\mathrm{~d}, J=1.8 \mathrm{~Hz}, 2 \mathrm{H}), 7.85(\mathrm{~d}, J=$ $8.7 \mathrm{~Hz}, 2 \mathrm{H}), 7.78(\mathrm{~d}, J=9.0 \mathrm{~Hz}, 2 \mathrm{H}), 7.74(\mathrm{dd}, J=9.0 \mathrm{~Hz}$ and $J=2.1 \mathrm{~Hz}, 2 \mathrm{H}), 1.49(\mathrm{~s}, 18 \mathrm{H})$. ${ }^{13} \mathrm{C} \mathrm{NMR}\left(100 \mathrm{MHz}, \mathrm{CDCl}_{3}\right) \delta 148.55,133.60,130.59,128.45,127.76,127.36,127.20,126.93$, 124.55, 124.13, 35.05, 31.71. Anal. calcd for $\mathrm{C}_{26} \mathrm{H}_{28}$ : C, 91.71; H, 8.29. Found: C, 91.69; H, 8.04 .

Corey-Fuchs Olefination of Xanthone. Xanthone (10, $1.984 \mathrm{~g}, 10.11 \mathrm{mmol})$, carbon tetrabromide $(6.189 \mathrm{~g}, 18.66 \mathrm{mmol})$, and triphenyl phosphine $(9.791 \mathrm{~g}, 37.33 \mathrm{mmol})$ were combined in $50 \mathrm{~mL}$ of anhydrous benzene as described in General Procedure 1. The reaction was run at $155^{\circ} \mathrm{C}$ for $45 \mathrm{~h}$. Column chromatography of the crude product on neutral alumina with hexanes as the eluent gave $2.334 \mathrm{~g}$ (66\% yield) of 9-(dibromomethylene)-9H-xanthene as an off-white powder that was pure by ${ }^{1} \mathrm{H}$ NMR analysis: mp $117-118{ }^{\circ} \mathrm{C}$. ${ }^{1} \mathrm{H} \mathrm{NMR}(400 \mathrm{MHz}$, $\left.\mathrm{CDCl}_{3}\right) \delta(\mathrm{ppm}): 7.98(\mathrm{dd}, J=8.0 \mathrm{~Hz}$ and $J=1.2 \mathrm{~Hz}, 2 \mathrm{H}), 7.34(\mathrm{ddd}, J=8.4 \mathrm{~Hz}, J=7.2 \mathrm{~Hz}$ and $J=1.6 \mathrm{~Hz}, 2 \mathrm{H}), 7.23(\mathrm{dd}, J=8.0 \mathrm{~Hz}$ and $J=0.8 \mathrm{~Hz}, 2 \mathrm{H}), 7.16(\mathrm{ddd}, J=7.6 \mathrm{~Hz}, J=7.8 \mathrm{~Hz}$ and $J$ $=1.4 \mathrm{~Hz}, 2 \mathrm{H}) .{ }^{13} \mathrm{C} \mathrm{NMR}\left(100 \mathrm{MHz}, \mathrm{CDCl}_{3}\right) \delta 152.86,131.74,129.46,128.25,123.78,122.68$, 116.72, 87.66. Anal. calcd for $\mathrm{C}_{14} \mathrm{H}_{8} \mathrm{Br}_{2} \mathrm{O}: \mathrm{C}, 47.77 ; \mathrm{H}, 2.29$. Found: C, 48.11; H, 1.96 .

Sonogashira Coupling of 9-(Dibromomethylene)-9H-xanthene. The 9(dibromomethylene)-9H-xanthene (1.018 g, $2.892 \mathrm{mmol}), \mathrm{PdCl}_{2}\left(\mathrm{PPh}_{3}\right)_{2}$ (177 mg, $0.252 \mathrm{mmol}$ ), copper(I) iodide $(100 \mathrm{mg}, 0.526 \mathrm{mmol})$, and trimethylsilyl acetylene $(1.75 \mathrm{~mL}, 12.4 \mathrm{mmol})$ were combined in $30 \mathrm{~mL}$ of triethylamine as described in General Procedure 2. The reaction was run at $90{ }^{\circ} \mathrm{C}$ for $44 \mathrm{~h}$. Column chromatography of the crude product on neutral alumina with a $2 \%$ ethyl acetate: $98 \%$ hexanes solution as the eluent gave $908 \mathrm{mg}$ (81\% yield) of 9[bis(trimethylsilylethynyl)methylene)-9H-xanthene as a dark brown solid that was pure by ${ }^{1} \mathrm{H}$ NMR analysis: $\mathrm{mp} 126-129{ }^{\circ} \mathrm{C}$. ${ }^{1} \mathrm{H}$ NMR $\left(300 \mathrm{MHz}, \mathrm{CDCl}_{3}\right) \delta(\mathrm{ppm}): 8.54(\mathrm{dd}, J=8.0 \mathrm{~Hz}$ and $J=1.4 \mathrm{~Hz}, 2 \mathrm{H}), 7.36(\mathrm{ddd}, J=8.1 \mathrm{~Hz}, J=7.4 \mathrm{~Hz}$ and $J=1.4 \mathrm{~Hz}, 2 \mathrm{H}), 7.21(\mathrm{dd}, J=8.0 \mathrm{~Hz}$ and $J$ $=1.4 \mathrm{~Hz}, 2 \mathrm{H}), 7.12(\mathrm{ddd}, J=8.2 \mathrm{~Hz}, J=7.0 \mathrm{~Hz}$ and $J=1.2 \mathrm{~Hz}, 2 \mathrm{H}), 0.24(\mathrm{~s}, 18 \mathrm{H}) .{ }^{13} \mathrm{C} \mathrm{NMR}$ $\left(100 \mathrm{MHz}_{\mathrm{CDCl}}\right) \delta 152.22,140.05,130.32,128.10,122.34,122.20,116.38,104.36,99.47$, 97.14, 0.065. HRMS ESI $(\mathrm{m} / \mathrm{z})$ : $[\mathrm{M}+\mathrm{Na}]^{+}$calcd for $\mathrm{C}_{24} \mathrm{H}_{26} \mathrm{NaSi}_{2} \mathrm{O}, 409.1420$. Found 409.1416.

Desilylation of 9-[bis(trimethylsilylethynyl)methylene)-9H-xanthene. $\quad$ The 9[bis (trimethylsilylethynyl)methylene)-9H-xanthene $(437 \mathrm{mg}, 1.13 \mathrm{mmol})$ and tetrabutylammonium fluoride trihydrate $(1.081 \mathrm{~g}, 3.426 \mathrm{mmol})$ were stirred in $20 \mathrm{~mL}$ of dichloromethane as described in General Procedure 3 until TLC showed no remaining starting material (ca $15 \mathrm{~min}$ ). Care was taken not to apply excessive heat during the workup, and an alumina plug was substituted for the plug of silica gel. The crude 9-(diethynylmethylene)-9Hxanthene was immediately subjected to the next reaction without further purification. ${ }^{1} \mathrm{H}$ NMR $\left(300 \mathrm{MHz}, \mathrm{CDCl}_{3}\right) \delta(\mathrm{ppm}): 8.50(\mathrm{dd}, J=8.0 \mathrm{~Hz}$ and $J=1.5 \mathrm{~Hz}, 2 \mathrm{H}), 7.40(\mathrm{ddd}, J=8.2 \mathrm{~Hz}, J=$ $7.2 \mathrm{~Hz}$ and $J=1.6 \mathrm{~Hz}, 2 \mathrm{H}), 7.25(\mathrm{dd}, J=8.4 \mathrm{~Hz}$ and $J=1.2 \mathrm{~Hz}, 2 \mathrm{H}), 7.18(\mathrm{ddd}, J=8.0 \mathrm{~Hz}, J=$ $7.2 \mathrm{~Hz}$ and $J=1.4 \mathrm{~Hz}, 2 \mathrm{H}), 3.28(\mathrm{~s}, 2 \mathrm{H})$.

Double Cyclization of 9-(Diethynylmethylene)-9H-xanthene. A solution of 9(diethynylmethylene)-9H-xanthene in 1,2-dichloroethane $(7 \mathrm{~mL}$ total volume) was added at a rate of $0.7 \mathrm{~mL} / \mathrm{h}$ with a syringe pump to a solution of $\mathrm{RuCl}_{2}\left(\mathrm{PPh}_{3}\right)\left(\eta^{6} \text {-para-cymene }\right)^{2}(136 \mathrm{mg}$, $0.240 \mathrm{mmol})$ and ammonium hexafluorophosphate $(82 \mathrm{mg}, 0.50 \mathrm{mmol})$ in $30 \mathrm{~mL}$ of $1,2-$ 
dichloroethane at $75{ }^{\circ} \mathrm{C}$ as described in General Procedure 4. Heating was continued for an additional $54 \mathrm{~h}$ after completion of the addition, for a total reaction time of $64 \mathrm{~h}$. Column chromatography of the crude product on silica gel with hexanes as the eluent gave $18 \mathrm{mg}$ of naphtho[2,1,8,7-klmn]xanthene (14) as a brownish solid (6\% yield over 2 steps) that had traces of an inseparable impurity by ${ }^{1} \mathrm{H}$ NMR analysis.

Alternate Double Cyclization of 9-(Diethynylmethylene)-9H-xanthene with $\mathbf{P t C l}_{2}$. A solution of 9-(diethynylmethylene)-9H-xanthene (47 $\mathrm{mg}, 0.12 \mathrm{mmol}$ ) prepared as described in General Procedure 3 in toluene (distilled over sodium; $5 \mathrm{~mL}$ total volume) was added at a rate of $0.25 \mathrm{~mL} / \mathrm{h}$ with a syringe pump to a solution of $\mathrm{PtCl}_{2}(19 \mathrm{mg}, 0.070 \mathrm{mmol})$ in $5 \mathrm{~mL}$ of toluene at $80{ }^{\circ} \mathrm{C}$ in a manner analogous to that described in General Procedure 4. Heating was continued for an additional $3 \mathrm{~h}$ after completion of the addition for a total reaction time of $23 \mathrm{~h}$. Column chromatography of the crude product on silica gel with hexanes as the eluent gave $4 \mathrm{mg}$ of naphtho[2,1,8,7-klmn]xanthene (14) as a yellow crystalline solid (13\% yield over 2 steps) that was pure by ${ }^{1} \mathrm{H}$ NMR analysis: $\mathrm{mp} 175-177{ }^{\circ} \mathrm{C}\left(\mathrm{lit}^{4} 177{ }^{\circ} \mathrm{C}\right){ }^{1} \mathrm{H}$ NMR $\left(500 \mathrm{MHz}, \mathrm{CDCl}_{3}\right) \delta$ (ppm): $7.70(\mathrm{~s}, 4 \mathrm{H}), 7.46-7.42(\mathrm{~m}, 4 \mathrm{H}), 7.06(\mathrm{dd}, J=6 \mathrm{~Hz}$ and $J=2.5 \mathrm{~Hz}, 2 \mathrm{H}) .{ }^{13} \mathrm{C}$ NMR $(125$ $\left.\mathrm{MHz}, \mathrm{CDCl}_{3}\right) \delta 152.82,132.89,128.28,126.69,125.70,124.24,122.66,120.37,120.15,109.75$. $\operatorname{HRMS}$ ESI $(\mathrm{m} / \mathrm{z}):[\mathrm{M}]^{+}$calcd for $\mathrm{C}_{18} \mathrm{H}_{10} \mathrm{O}, 242.0732$. Found 242.0734.

Corey-Fuchs Olefination of 9,10-Anthraquinone. Anthraquinone (15, $206 \mathrm{mg}, 0.989$ $\mathrm{mmol})$, carbon tetrabromide $(1.025 \mathrm{~g}, 3.091 \mathrm{mmol})$, and triphenyl phosphine $(1.638 \mathrm{~g}, 6.245$ mmol) were combined in $15 \mathrm{~mL}$ of anhydrous dichloromethane at $0{ }^{\circ} \mathrm{C}$. The reaction was allowed to warm to room temperature for $24 \mathrm{~h}$. Column chromatography of the crude product on silica gel with hexanes as the eluent gave $510 \mathrm{mg}$ (99\% yield) of 9,10-bis(dibromomethylene)9,10-dihydroanthracene as a white powder) that was pure by ${ }^{1} \mathrm{H}$ NMR analysis. ${ }^{1} \mathrm{H}$ NMR (300 $\left.\mathrm{MHz} \mathrm{CDCl}_{3}\right) \delta(\mathrm{ppm}): 7.84\left(\mathrm{AA}^{\prime}\right.$ of $\left.\mathrm{AA}^{\prime} \mathrm{BB}^{\prime}, 4 \mathrm{H}\right), 7.29$ (BB' of AA'BB', 4H). Literature NMR. ${ }^{5}$

Sonogashira Coupling of 9,10-bis(dibromomethylene)-9,10-dihydroanthracene. The 9,10bis(dibromomethylene)-9,10-dihydroanthracene (530 g, $1.02 \mathrm{mmol}), \mathrm{PdCl}_{2}\left(\mathrm{PPh}_{3}\right)_{2}(101 \mathrm{mg}$, $0.144 \mathrm{mmol})$, copper(I) iodide $(64 \mathrm{mg}, 0.34 \mathrm{mmol})$, and trimethylsilyl acetylene $(2.0 \mathrm{~mL}, 14$ mmol) were combined in $30 \mathrm{~mL}$ of triethylamine as described in General Procedure 2. The reaction was run at $90{ }^{\circ} \mathrm{C}$ for $16 \mathrm{~h}$. Column chromatography of the crude product on silica gel with a 2\% ethyl acetate: $98 \%$ hexanes solution as the eluent gave $596 \mathrm{mg}$ (99\% yield) of 9,10bis[bis(trimethylsilylethynyl)methylene]-9,10-dihydroanthracene as an orange-brown solid that was pure by ${ }^{1} \mathrm{H} \mathrm{NMR}$ analysis. ${ }^{1} \mathrm{H} \mathrm{NMR}\left(300 \mathrm{MHz}, \mathrm{CDCl}_{3}\right) \delta(\mathrm{ppm}): 8.28$ (AA' of AA'BB', 4H), 7.26 (BB' of AA'BB', 4H), 0.22 (s, 36H). Literature NMR. ${ }^{5}$

Desilylation of 9,10-bis[bis(trimethylsilylethynyl)methylene]-9,10-dihydroanthracene. The 9,10-bis[bis(trimethylsilylethynyl)methylene]-9,10-dihydroanthracene (105 $\mathrm{mg}, 0.179$ $\mathrm{mmol})$ and tetrabutylammonium fluoride trihydrate $(233 \mathrm{mg}, 0.737 \mathrm{mmol})$ were stirred in $3 \mathrm{~mL}$ of dichloromethane at $0{ }^{\circ} \mathrm{C}$ as described in General Procedure 3 until TLC showed no remaining starting material (ca 25 min). Care was taken not to apply excessive heat during the workup.

4. Kruber, O. Chem. Ber. 1941, 74B, 1688-1692.

5. Neidlein, R.; Winter, M. Synthesis 1998, 9, 1362-1366. 
The crude 9,10-bis[bis(ethynyl)methylene]-9,10-dihydroanthracene was immediately subjected to the next reaction without further purification. ${ }^{1} \mathrm{H}$ NMR $\left(400 \mathrm{MHz}, \mathrm{CDCl}_{3}\right) \delta(\mathrm{ppm}): 8.24$ (AA' of AA'BB', 4H), 7.35 (BB' of AA'BB', 4H), 3.27 (s, 4H).

Quadruple Cyclization of 9,10-bis[bis(ethynyl)methylene]-9,10-dihydroanthracene. A solution of 9,10-bis[bis(ethynyl)methylene]-9,10-dihydroanthracene ( $2.5 \mathrm{~mL}$ total volume) was added at a rate of $0.25 \mathrm{~mL} / \mathrm{h}$ with a syringe pump to a solution of $\mathrm{RuCl}_{2}\left(\mathrm{PPh}_{3}\right)\left(\eta^{6} \text {-para-cymene }\right)^{2}(24 \mathrm{mg}, 0.042 \mathrm{mmol})$ and ammonium hexafluorophosphate (16 $\mathrm{mg}, 0.098 \mathrm{mmol}$ ) in $17 \mathrm{~mL}$ of 1,2-dichloroethane at $75^{\circ} \mathrm{C}$ as described in General Procedure 4. Heating was continued for an additional $7 \mathrm{~h}$ after completion of the addition, for a total reaction time of $17 \mathrm{~h}$. The reaction mixture was filtered through a short pad of silica gel, which was washed with several additional portions of dichloromethane. Removal of the solvent on a rotary evaporator gave $8 \mathrm{mg}$ of coronene (19) as a yellow solid (15\% yield over 2 steps) that was pure by ${ }^{1} \mathrm{H}$ NMR analysis. The ${ }^{1} \mathrm{H}$ NMR spectrum matched that reported in the literature. ${ }^{6}$

Corey-Fuchs Olefination of 9-Fluorenone. Fluorenone (20, $1.022 \mathrm{~g}, 5.673 \mathrm{mmol})$, carbon tetrabromide $(3.400 \mathrm{~g}, 10.25 \mathrm{mmol})$, and triphenyl phosphine $(5.378 \mathrm{~g}, 20.50 \mathrm{mmol})$ were combined in $50 \mathrm{~mL}$ of anhydrous dichloromethane. The reaction was run at $40{ }^{\circ} \mathrm{C}$ for $23 \mathrm{~h}$. Column chromatography of the crude product on silica gel with hexanes as the eluent gave 1.305 $\mathrm{g}(68 \%$ yield) of 9-(dibromomethylene)-9H-fluorene as a yellow crystalline solid that was pure by ${ }^{1} \mathrm{H}$ NMR analysis. The ${ }^{1} \mathrm{H}$ NMR spectrum matched that reported in the literature. ${ }^{7}{ }^{13} \mathrm{C}$ NMR $\left(100 \mathrm{MHz}, \mathrm{CDCl}_{3}\right) \delta 147.21,140.50,138.12,129.48,127.41,126.05,119.65,90.78$.

Sonogashira Coupling of 9-(dibromomethylene)-9H-fluorene. The 9-(dibromomethylene)9H-fluorene (352 mg, $1.05 \mathrm{mmol}), \mathrm{PdCl}_{2}\left(\mathrm{PPh}_{3}\right)_{2}(77 \mathrm{mg}, 0.11 \mathrm{mmol})$, copper(I) iodide (47 mg, $0.25 \mathrm{mmol})$, and trimethylsilyl acetylene $(0.83 \mathrm{~mL}, 5.9 \mathrm{mmol})$ were combined in $20 \mathrm{~mL}$ of triethylamine as described in General Procedure 2. The reaction was run at $90{ }^{\circ} \mathrm{C}$ for $18 \mathrm{~h}$. Column chromatography of the crude product on silica gel with hexanes as the eluent gave 260 $\mathrm{mg}$ (67\% yield) of 9-[bis(trimethylsilylethynyl)methylene]-9H-fluorene as a brown solid that was pure by ${ }^{1} \mathrm{H}$ NMR analysis. The ${ }^{1} \mathrm{H}$ NMR spectrum matched that reported in the literature. ${ }^{5}$ ${ }^{13} \mathrm{C}$ NMR $\left(100 \mathrm{MHz}, \mathrm{CDCl}_{3}\right) \delta 146.89,140.26,137.42,129.80,127.31,125.98,119.56,104.70$, $103.48,101.03,0.13$.

Desilylation of 9-[bis(trimethylsilylethynyl)methylene]-9H-fluorene. The 9-[bis(trimethylsilylethynyl)methylene]-9H-fluorene $(64 \mathrm{mg}, 0.17 \mathrm{mmol})$ and tetrabutylammonium fluoride trihydrate $(117 \mathrm{mg}, 0.448 \mathrm{mmol})$ were stirred in $11 \mathrm{~mL}$ of dichloromethane as described in General Procedure 3 until TLC showed no remaining starting material (ca $30 \mathrm{~min}$ ). Care was taken not to apply excessive heat during the workup. The crude 9-bis(ethynyl)methylene-9Hfluorene was immediately subjected to the next reaction without further purification. ${ }^{1} \mathrm{H}$ NMR $\left(300 \mathrm{MHz}, \mathrm{CDCl}_{3}\right) \delta(\mathrm{ppm}): 8.64(\mathrm{~d}, J=7.8 \mathrm{~Hz}, 2 \mathrm{H}), 7.64(\mathrm{~d}, J=7.5 \mathrm{~Hz}, 2 \mathrm{H}), 7.38(\mathrm{td}, J=7.4$ $\mathrm{Hz}$ and $J=1.0 \mathrm{~Hz}, 2 \mathrm{H}), 7.28$ (td, $J=7.8 \mathrm{~Hz}$ and $J=1.4 \mathrm{~Hz}, 2 \mathrm{H}), 3.76(\mathrm{~s}, 2 \mathrm{H})$.

6. Westermayer, M.; Hafelinger, G.; Regelmann, C. Tetrahedron 1984, 40, 1845-1854.

7. Paul, G. C.; Gajewski, J. J. Synthesis 1997, 5, 524-526. 
ja038254I - Supporting Information

Donovan and Scott
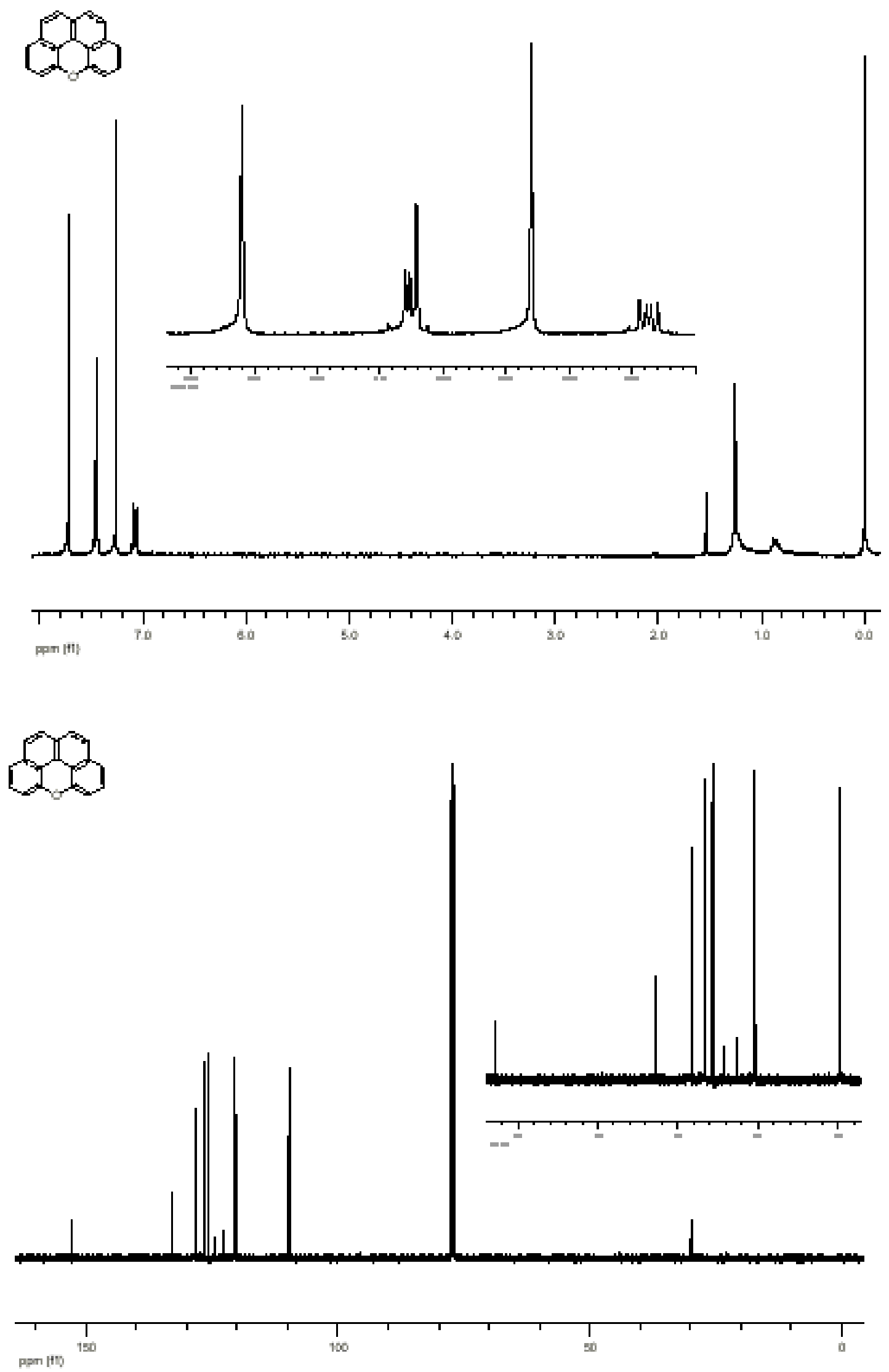

page S6 

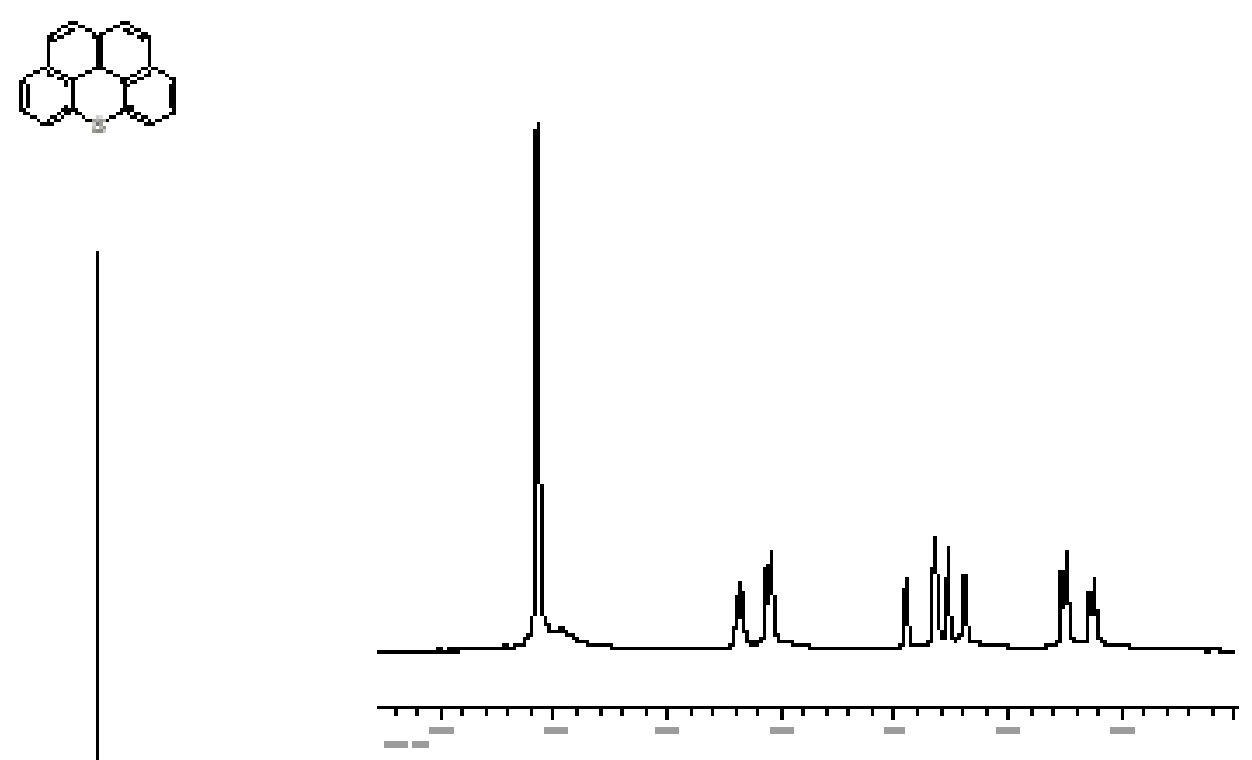

illi

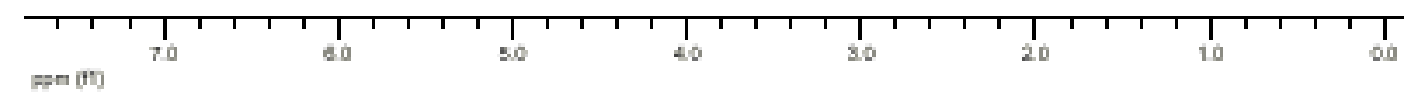

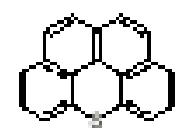
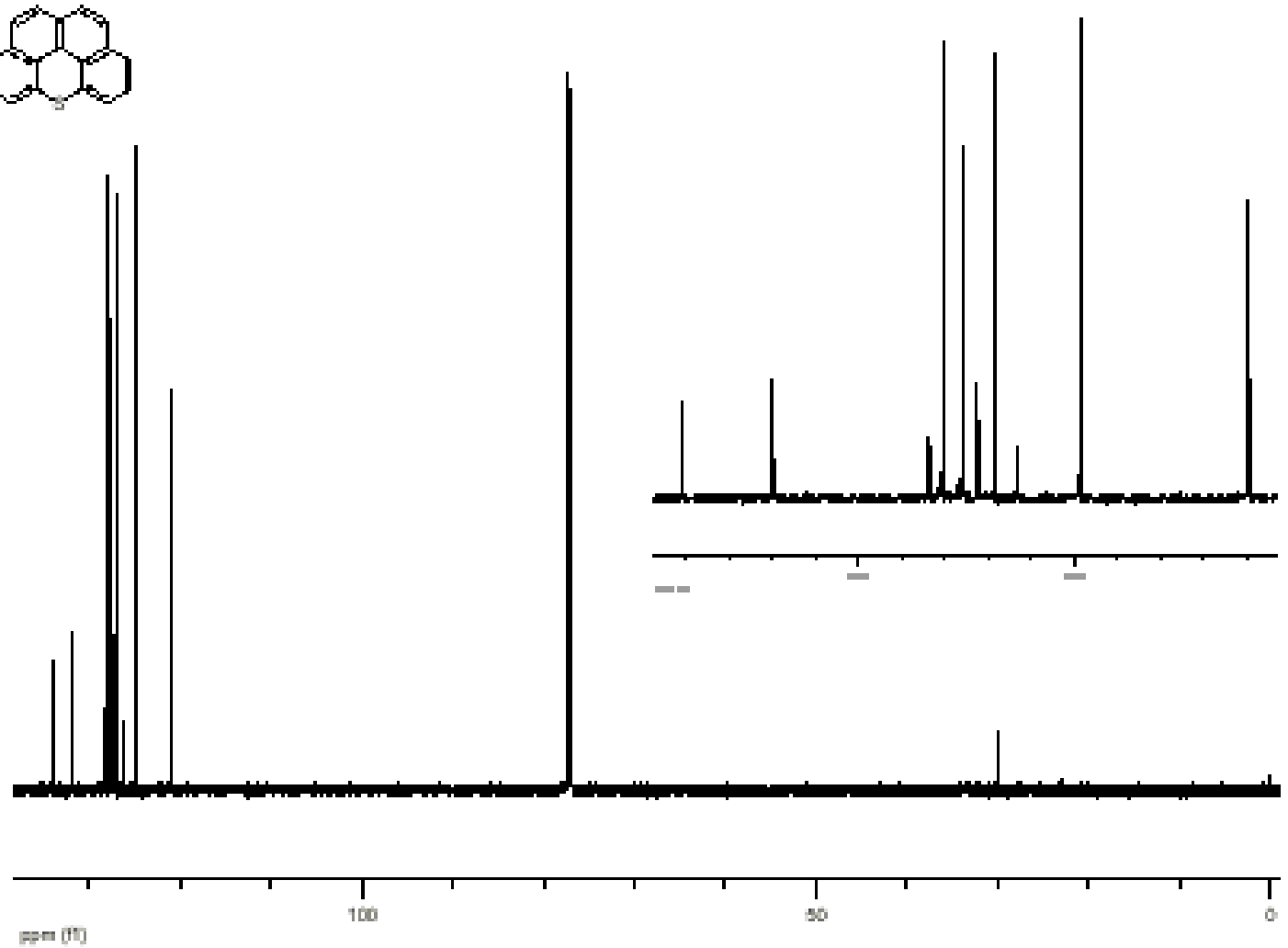

page $\mathrm{S} 7$ 


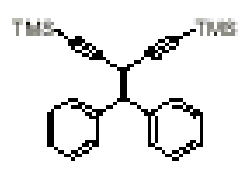

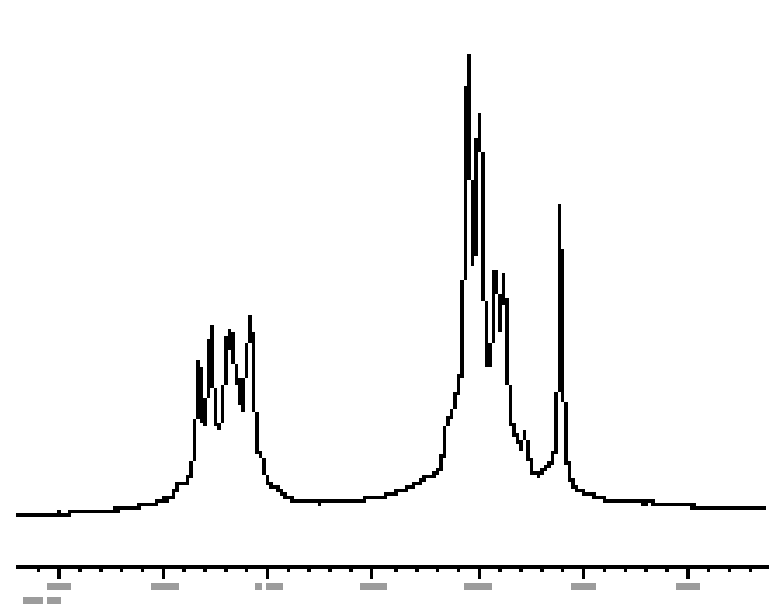

$\mu$
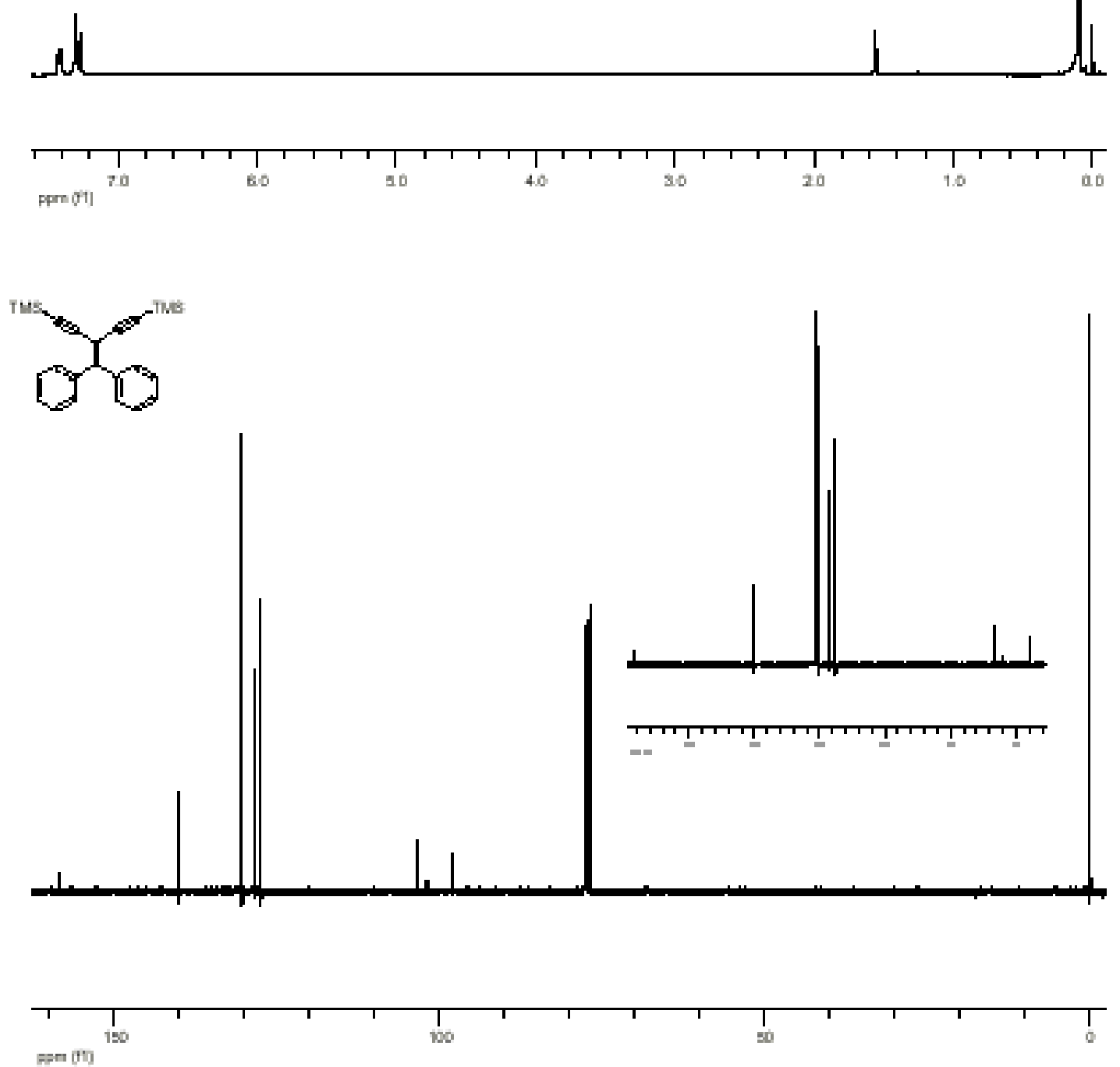

page $\mathrm{S} 8$ 


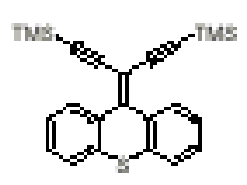

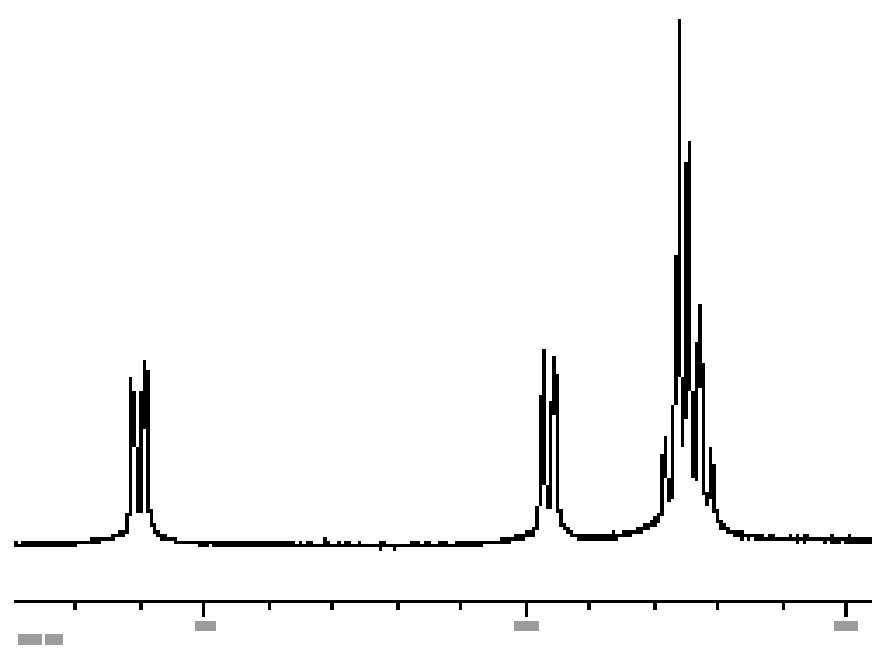

L

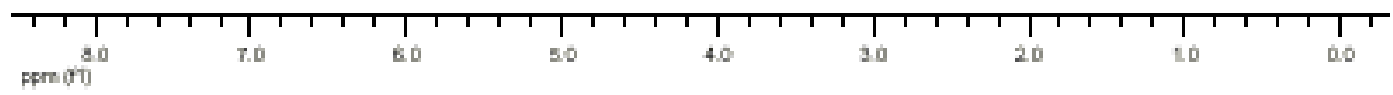

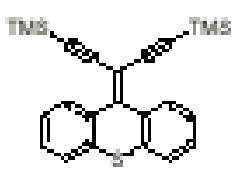
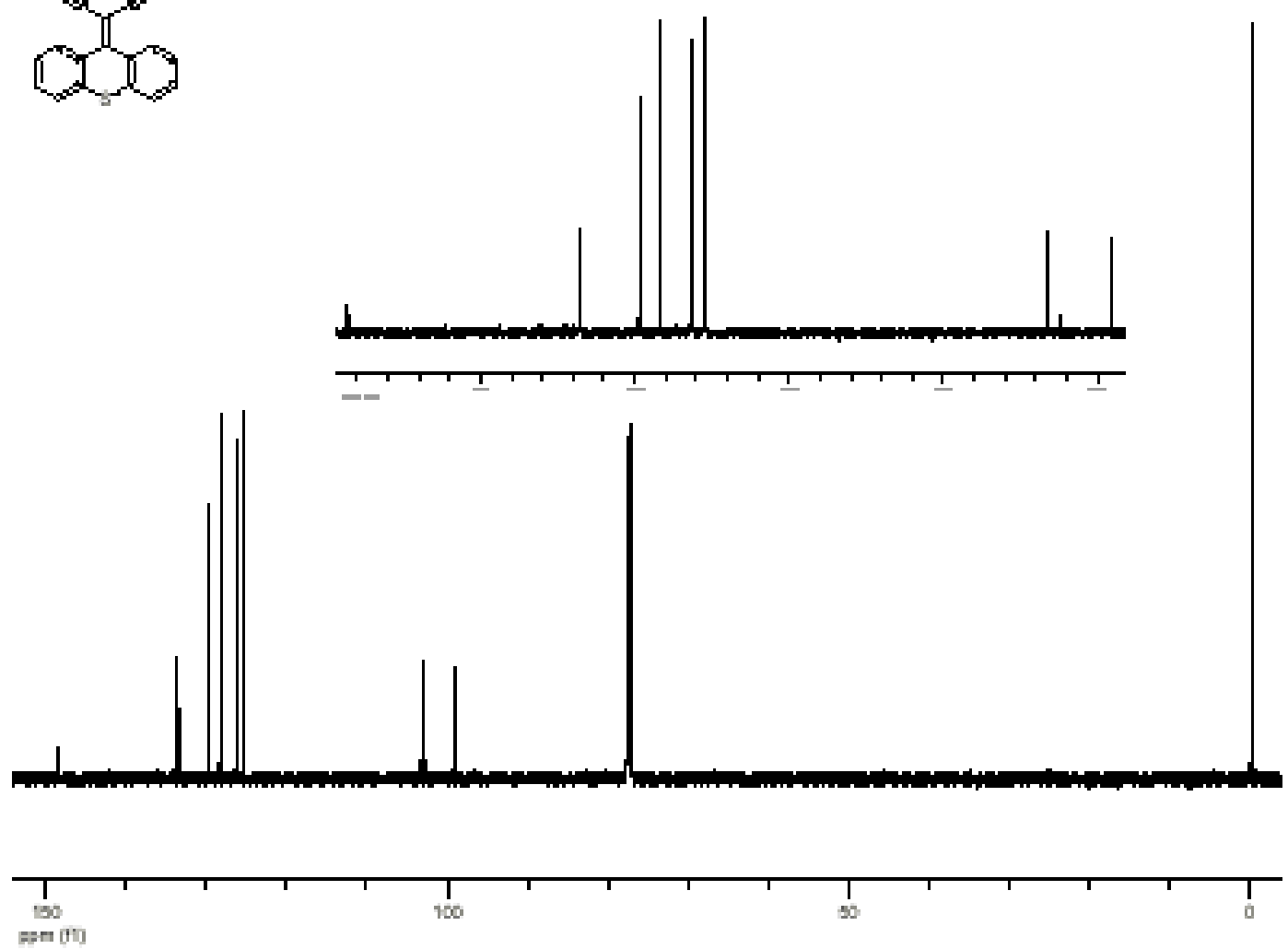

page $\mathrm{S} 9$ 

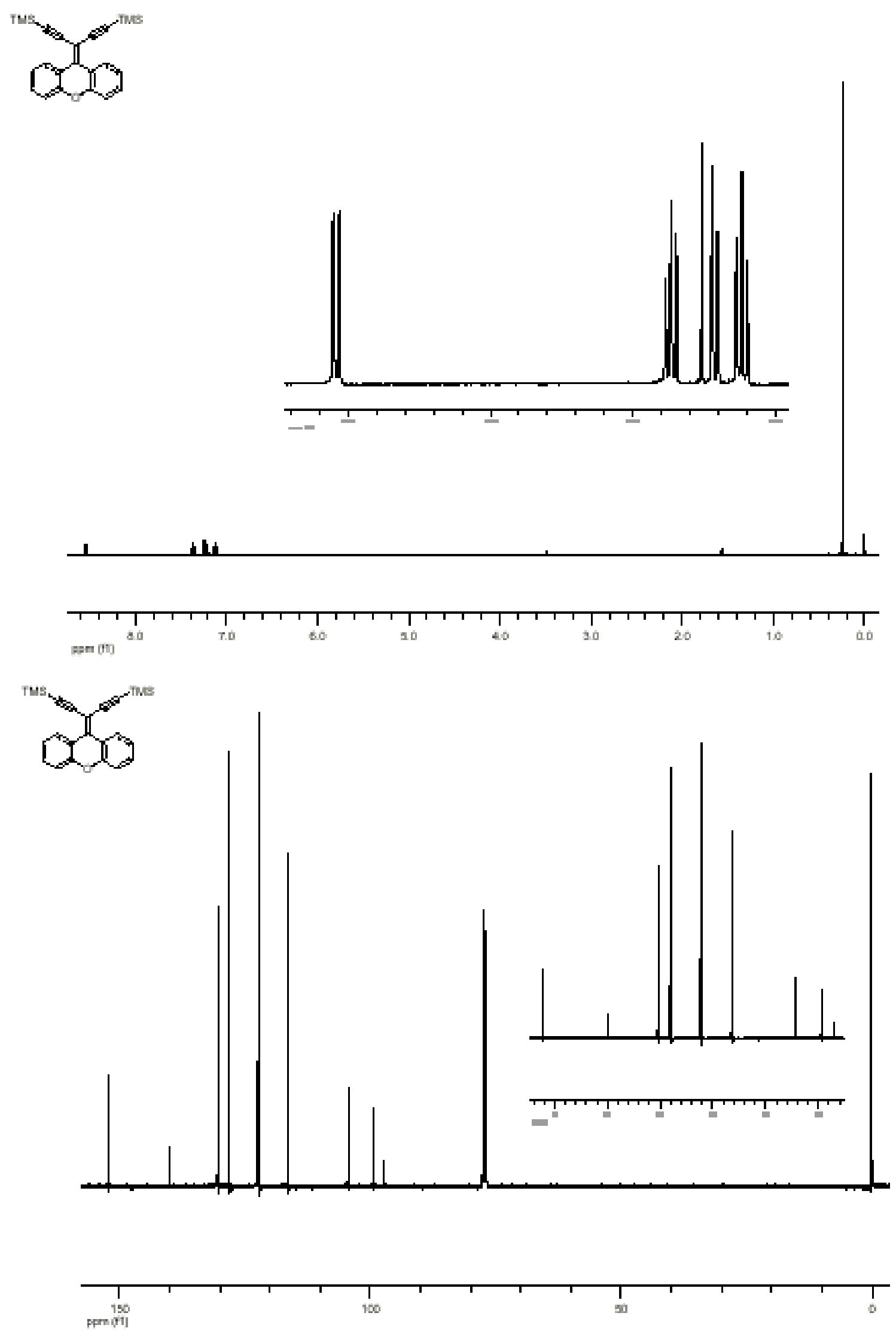

page $\mathrm{S} 10$ 


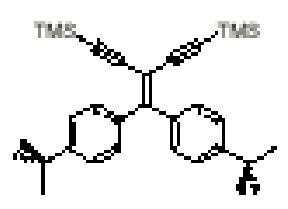
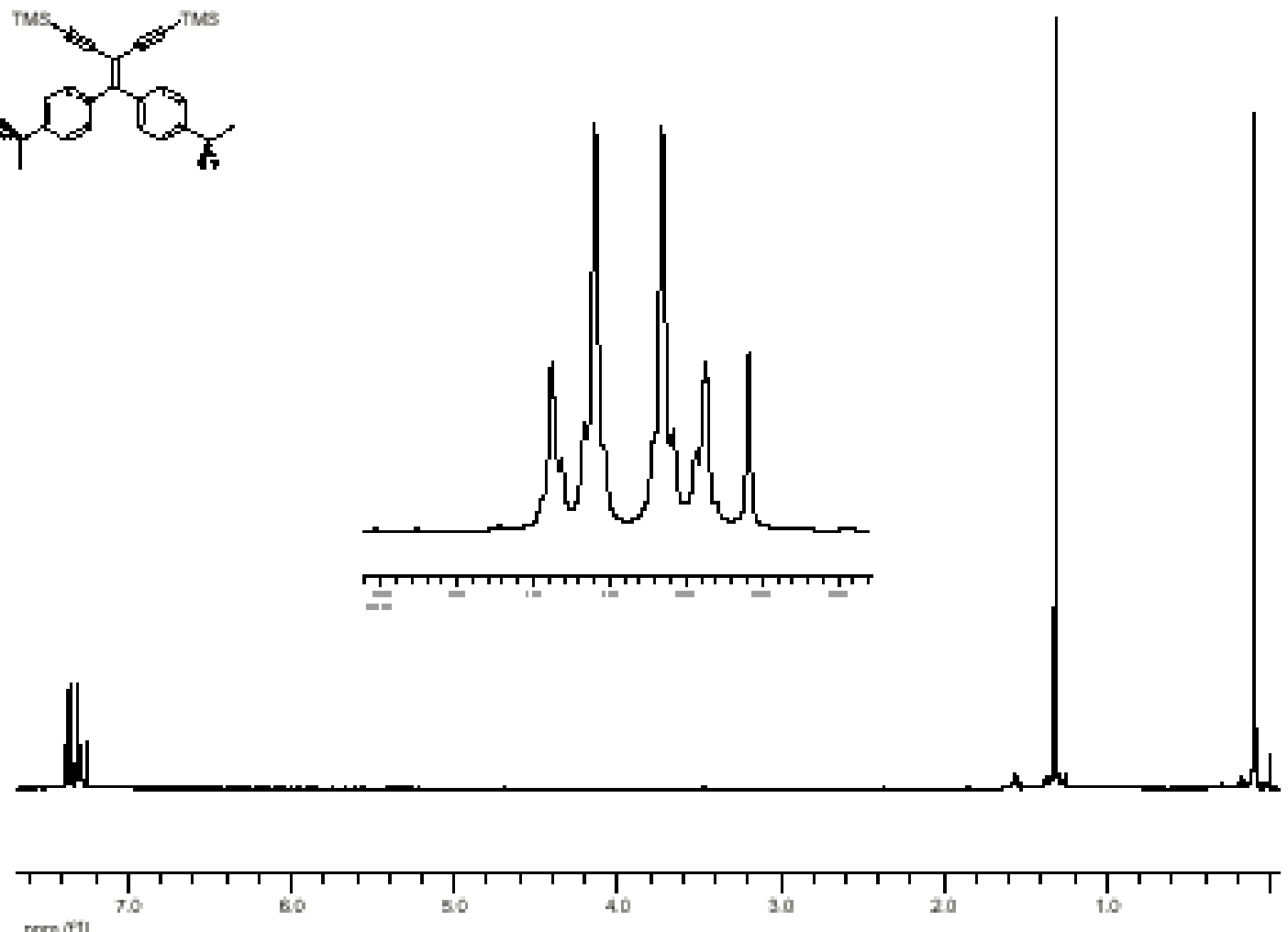
ppra oi1]
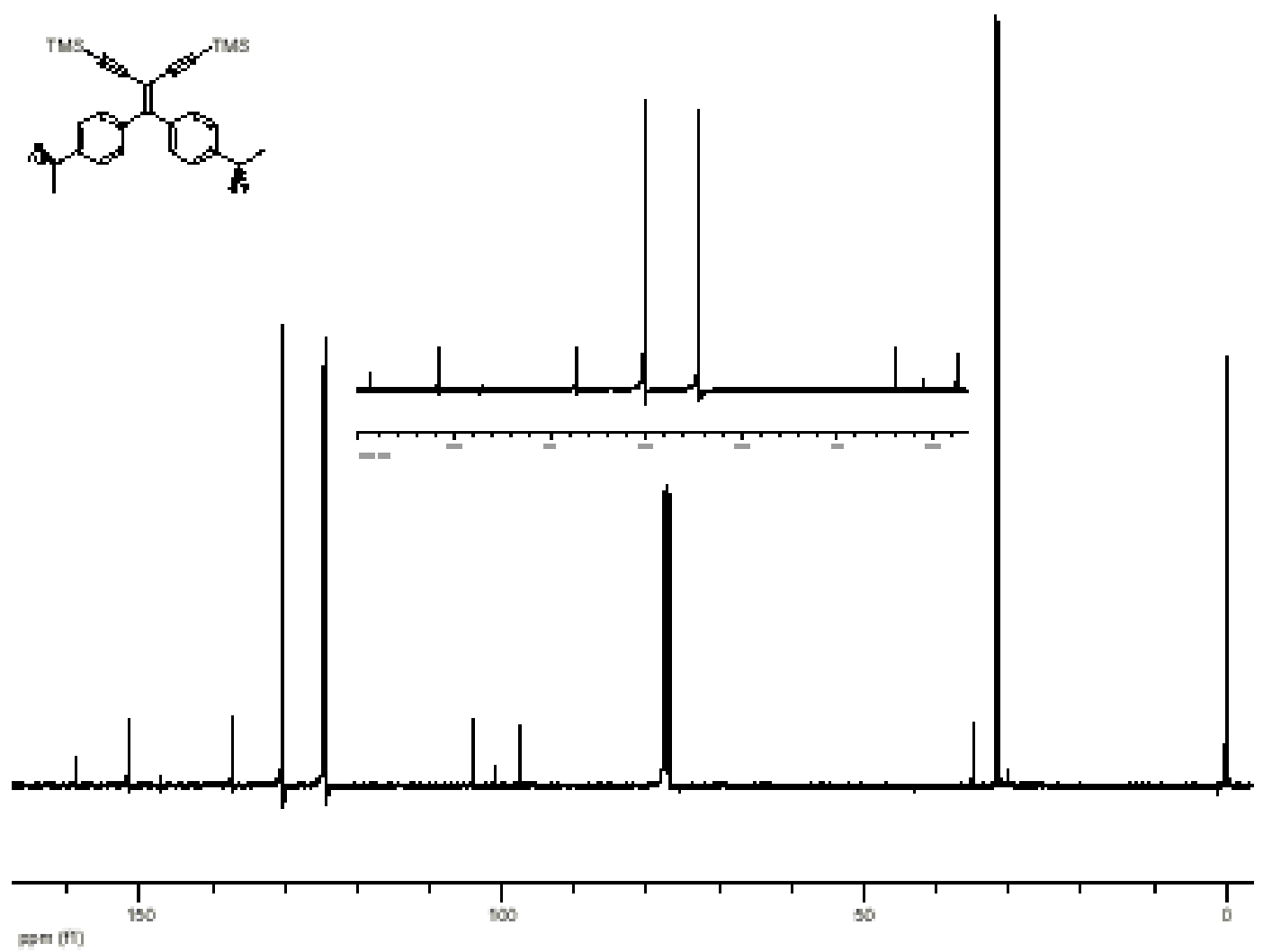


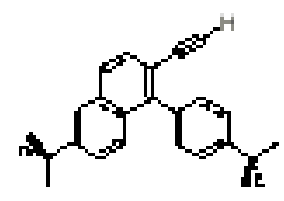
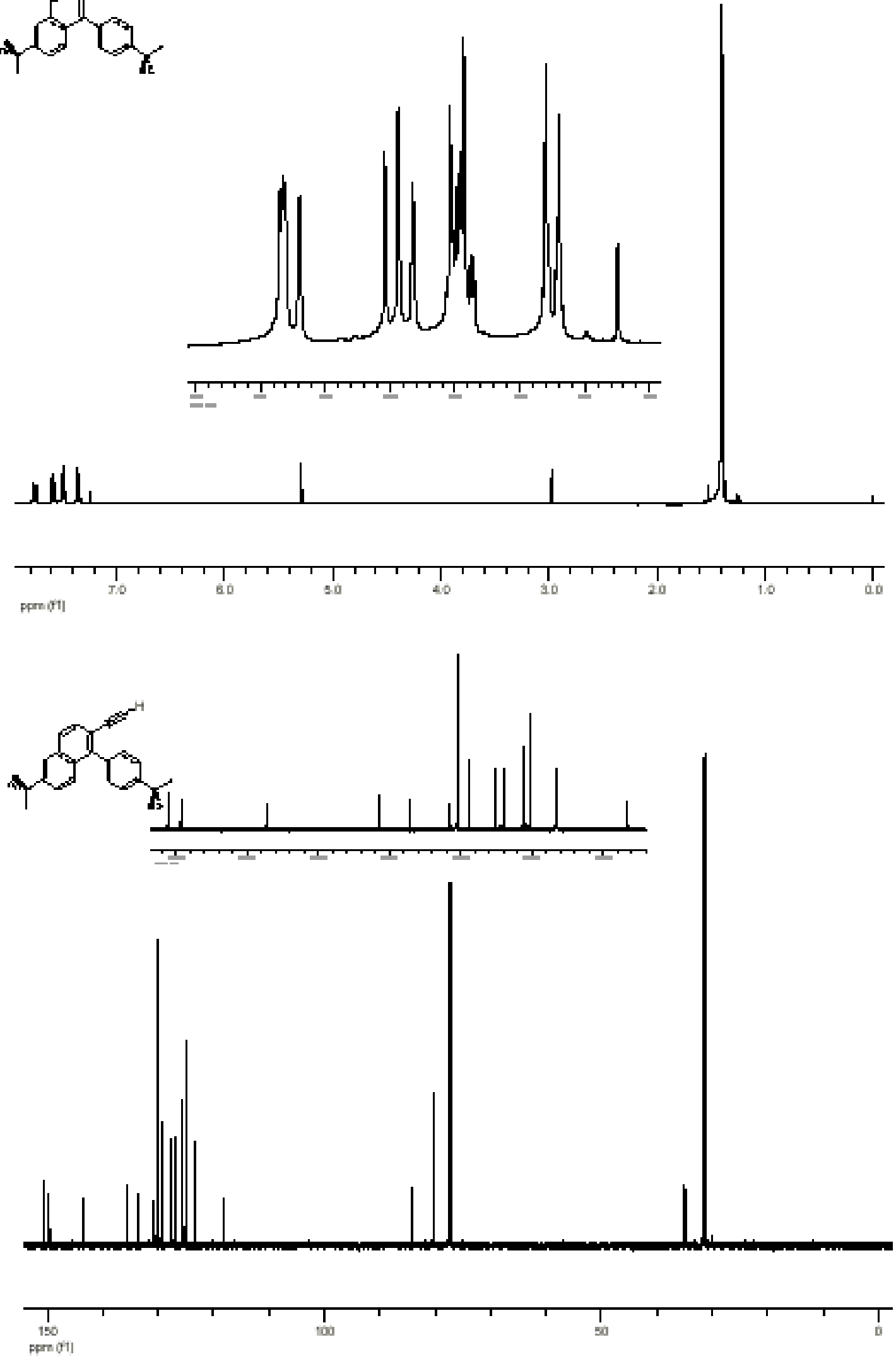

page $\mathrm{S} 12$ 

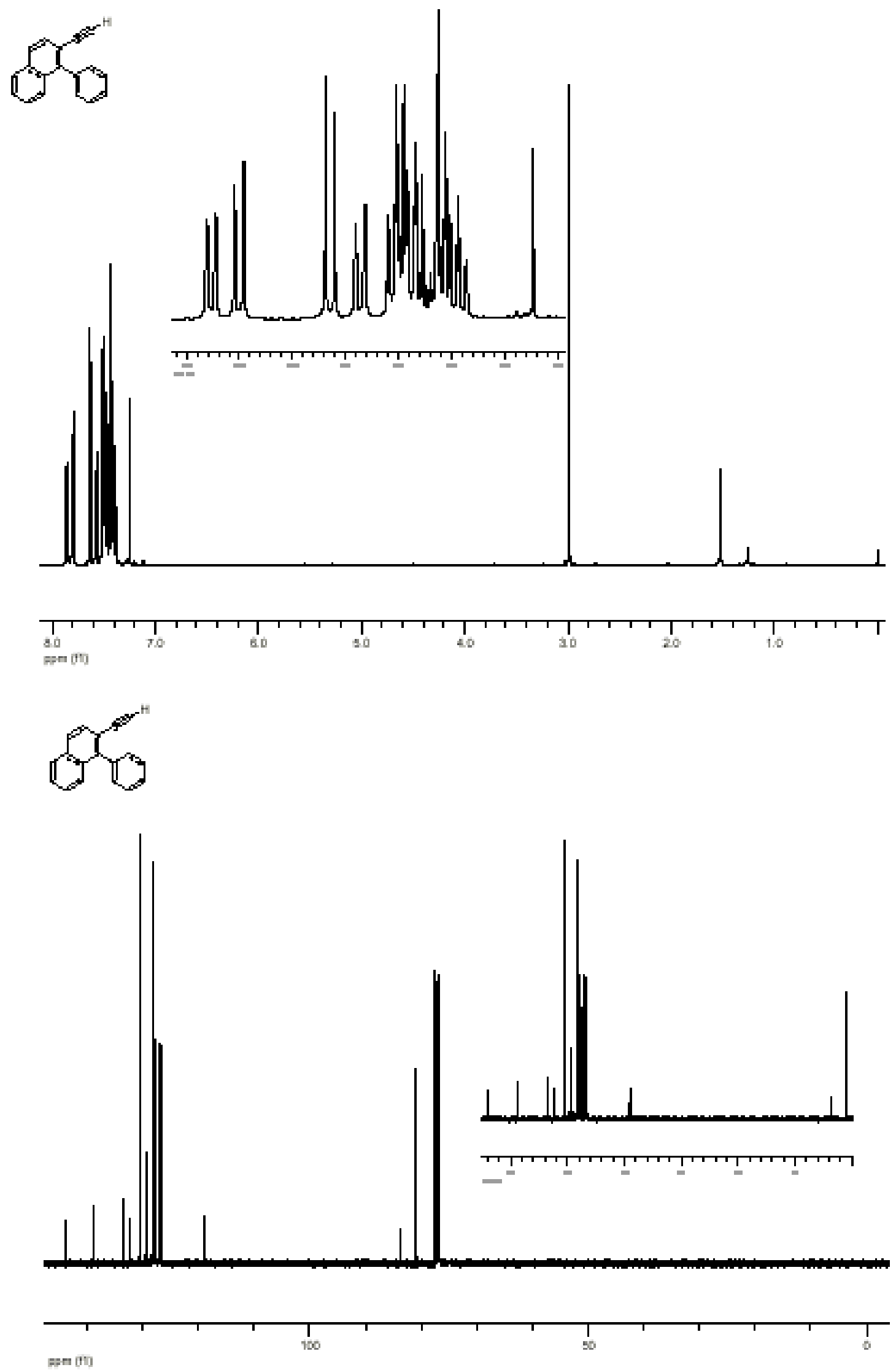

page $\mathrm{S} 13$ 
X-Ray Data for naphtho[2,1,8,7-klmn]thioxanthene (2):
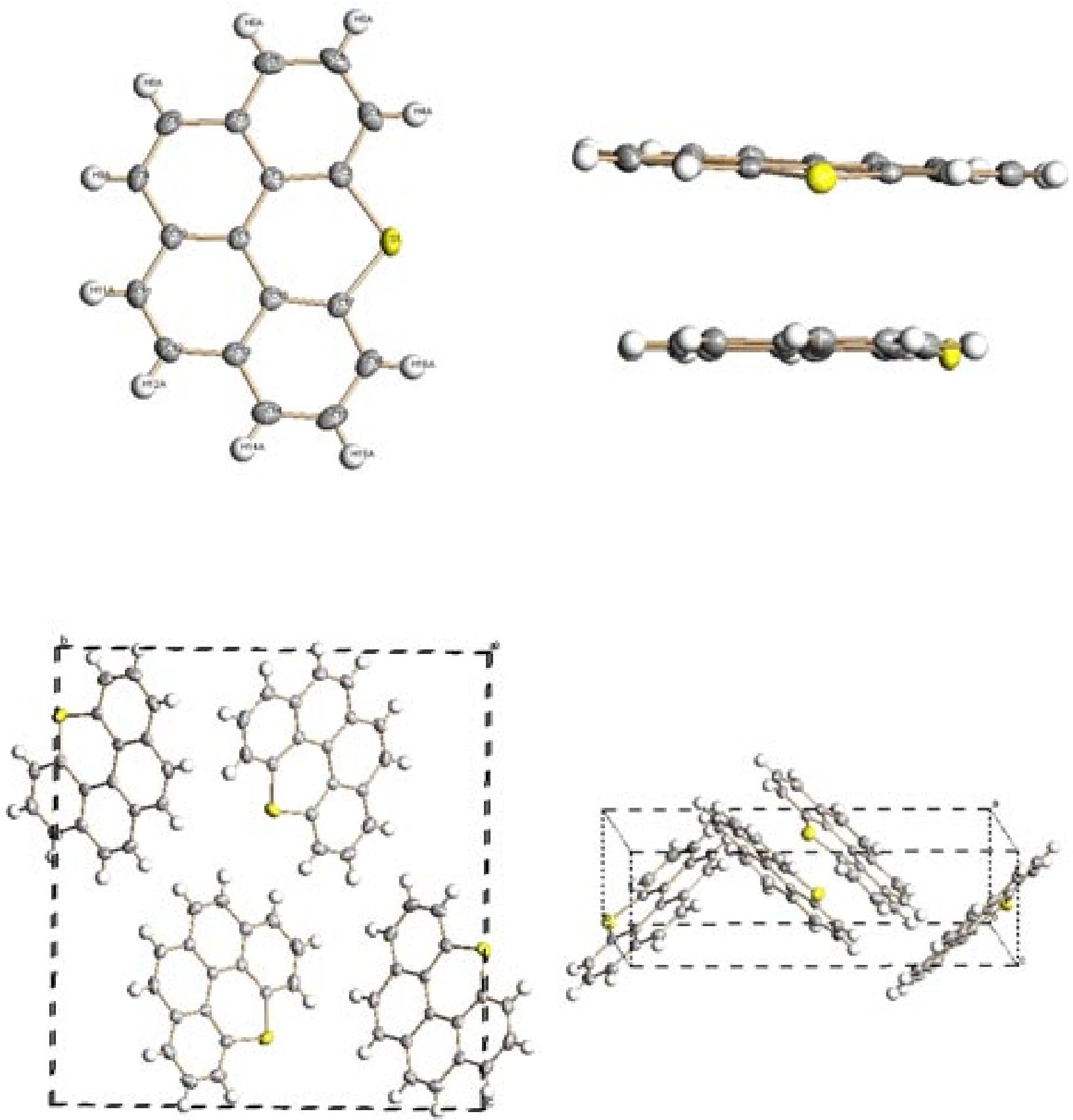

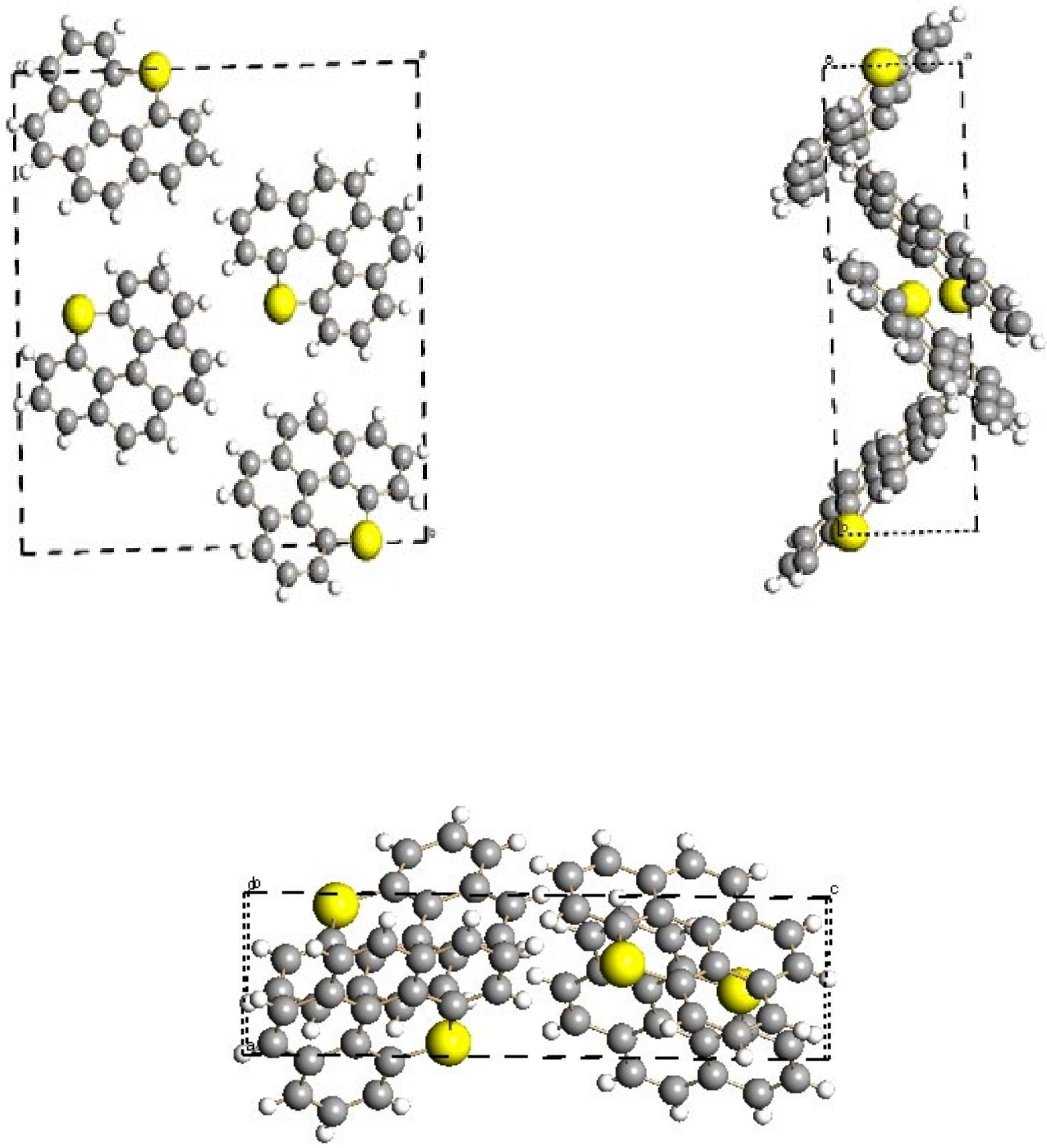
Table 1. Crystal data and structure refinement for naphtho[2,1,8,7-klmn]thioxanthene (2).

Identification code

Empirical formula

Formula weight

Temperature

Wavelength

Crystal system

Space group

Unit cell dimensions

Volume

Z

Density (calculated)

Absorption coefficient

$\mathrm{F}(000)$

Crystal size

Theta range for data collection

Index ranges

Reflections collected

Independent reflections

Completeness to theta $=28.31^{\circ}$

Absorption correction

Refinement method

Data / restraints / parameters

Goodness-of-fit on $\mathrm{F}^{2}$

Final R indices [I $>2$ sigma(I)]

$\mathrm{R}$ indices (all data)

Absolute structure parameter

Largest diff. peak and hole pat $3 \mathrm{a} 4 \mathrm{t}$

C18 H10 S

258.32

193(2) K

$0.71073 \AA$

Orthorhombic

P2(1)2(1)2(1)

$\mathrm{a}=4.6014(4) \AA$

$\mathrm{b}=15.5555(13) \AA$

$\alpha=90^{\circ}$.

$\mathrm{c}=16.5551(13) \AA$

$\beta=90^{\circ}$.

$1184.97(17) \AA^{3}$

4

$1.448 \mathrm{Mg} / \mathrm{m}^{3}$

$0.252 \mathrm{~mm}^{-1}$

536

$0.65 \times 0.10 \times 0.10 \mathrm{~mm}^{3}$

1.80 to $28.31^{\circ}$.

$-6<=\mathrm{h}<=6,-20<=\mathrm{k}<=19,-19<=1<=22$

8732

$2930[\mathrm{R}(\mathrm{int})=0.0731]$

$99.5 \%$

None

Full-matrix least-squares on $\mathrm{F}^{2}$

$2930 / 0 / 172$

1.004

$\mathrm{R} 1=0.0417, \mathrm{wR} 2=0.0932$

$\mathrm{R} 1=0.0468, \mathrm{wR} 2=0.0958$

$-0.03(8)$

0.416 and -0.209 e. $\AA^{-3}$

Table 2. Atomic coordinates $\left(\mathrm{x} 10^{4}\right)$ and equivalent isotropic displacement parameters $\left(\AA^{2} \times 10^{3}\right)$ for naphtho[2,1,8,7-klmn]thioxanthene (2). U(eq) is defined as one third of the trace of the orthogonalized $\mathrm{U}^{\mathrm{ij}}$ tensor.

\begin{tabular}{lrrrr}
\hline & $\mathrm{x}$ & $\mathrm{y}$ & $\mathrm{z}$ & $\mathrm{U}(\mathrm{eq})$ \\
\hline $\mathrm{S}(1)$ & & & & \\
$\mathrm{C}(18)$ & $800(1)$ & $-52(1)$ & $1506(1)$ & $33(1)$ \\
$\mathrm{C}(10)$ & $725(4)$ & $-644(1)$ & $3094(1)$ & $22(1)$ \\
$\mathrm{C}(2)$ & $3836(4)$ & $-1833(1)$ & $3544(1)$ & $24(1)$ \\
$\mathrm{C}(17)$ & $3981(4)$ & $-1436(1)$ & $2114(1)$ & $22(1)$ \\
$\mathrm{C}(7)$ & $-376(4)$ & $-62(1)$ & $2507(1)$ & $25(1)$ \\
$\mathrm{C}(8)$ & $6030(4)$ & $-2101(1)$ & $1975(1)$ & $26(1)$ \\
$\mathrm{C}(1)$ & $6988(4)$ & $-2616(1)$ & $2639(1)$ & $30(1)$ \\
$\mathrm{C}(9)$ & $2848(4)$ & $-1296(1)$ & $2913(1)$ & $22(1)$ \\
$\mathrm{C}(3)$ & $5927(4)$ & $-2484(1)$ & $3385(1)$ & $28(1)$ \\
$\mathrm{C}(6)$ & $3119(4)$ & $-939(1)$ & $1430(1)$ & $26(1)$ \\
$\mathrm{C}(13)$ & $7129(4)$ & $-2254(1)$ & $1192(1)$ & $33(1)$ \\
$\mathrm{C}(4)$ & $-370(4)$ & $-564(1)$ & $3895(1)$ & $26(1)$ \\
$\mathrm{C}(14)$ & $4154(4)$ & $-1126(1)$ & $669(1)$ & $33(1)$ \\
$\mathrm{C}(16)$ & $-2479(4)$ & $63(1)$ & $4079(1)$ & $32(1)$ \\
$\mathrm{C}(11)$ & $-2432(4)$ & $547(1)$ & $2712(1)$ & $32(1)$ \\
$\mathrm{C}(5)$ & $2722(4)$ & $-1721(1)$ & $4341(1)$ & $30(1)$ \\
$\mathrm{C}(12)$ & $6168(5)$ & $-1778(1)$ & $550(1)$ & $35(1)$ \\
$\mathrm{C}(15)$ & $710(4)$ & $-1121(1)$ & $4510(1)$ & $30(1)$ \\
& $-3482(4)$ & $612(1)$ & $3493(1)$ & $35(1)$ \\
\hline
\end{tabular}


Table 3. Bond lengths $[\AA]$ and angles $\left[^{\circ}\right]$ for naphtho[2,1,8,7-klmn $]$ thioxanthene (2).

\begin{tabular}{|c|c|c|c|}
\hline$S(1)-C(17)$ & $1.7432(17)$ & $\mathrm{C}(17)-\mathrm{S}(1)-\mathrm{C}(3)$ & $104.53(8)$ \\
\hline $\mathrm{S}(1)-\mathrm{C}(3)$ & $1.7484(18)$ & $\mathrm{C}(17)-\mathrm{C}(18)-\mathrm{C}(13)$ & $117.04(16)$ \\
\hline $\mathrm{C}(18)-\mathrm{C}(17)$ & $1.422(2)$ & $\mathrm{C}(17)-\mathrm{C}(18)-\mathrm{C}(1)$ & $123.21(15)$ \\
\hline $\mathrm{C}(18)-\mathrm{C}(13)$ & $1.423(2)$ & $\mathrm{C}(13)-\mathrm{C}(18)-\mathrm{C}(1)$ & $119.75(15)$ \\
\hline $\mathrm{C}(18)-\mathrm{C}(1)$ & $1.439(2)$ & $\mathrm{C}(1)-\mathrm{C}(10)-\mathrm{C}(9)$ & $120.15(16)$ \\
\hline $\mathrm{C}(10)-\mathrm{C}(1)$ & $1.412(2)$ & $C(1)-C(10)-C(11)$ & $119.72(16)$ \\
\hline $\mathrm{C}(10)-\mathrm{C}(9)$ & $1.421(2)$ & $\mathrm{C}(9)-\mathrm{C}(10)-\mathrm{C}(11)$ & $120.13(15)$ \\
\hline $\mathrm{C}(10)-\mathrm{C}(11)$ & $1.427(2)$ & $\mathrm{C}(7)-\mathrm{C}(2)-\mathrm{C}(3)$ & $116.80(15)$ \\
\hline $\mathrm{C}(2)-\mathrm{C}(7)$ & $1.419(2)$ & $\mathrm{C}(7)-\mathrm{C}(2)-\mathrm{C}(1)$ & $120.02(15)$ \\
\hline$C(2)-C(3)$ & $1.427(2)$ & $\mathrm{C}(3)-\mathrm{C}(2)-\mathrm{C}(1)$ & $123.18(16)$ \\
\hline $\mathrm{C}(2)-\mathrm{C}(1)$ & $1.439(2)$ & $\mathrm{C}(16)-\mathrm{C}(17)-\mathrm{C}(18)$ & $120.83(16)$ \\
\hline$C(17)-C(16)$ & $1.381(2)$ & $C(16)-C(17)-S(1)$ & $116.13(13)$ \\
\hline $\mathrm{C}(7)-\mathrm{C}(6)$ & $1.411(2)$ & $\mathrm{C}(18)-\mathrm{C}(17)-\mathrm{S}(1)$ & $123.03(13)$ \\
\hline $\mathrm{C}(7)-\mathrm{C}(8)$ & $1.431(2)$ & $C(6)-C(7)-C(2)$ & $120.68(16)$ \\
\hline $\mathrm{C}(8)-\mathrm{C}(9)$ & $1.343(3)$ & $\mathrm{C}(6)-\mathrm{C}(7)-\mathrm{C}(8)$ & $120.07(17)$ \\
\hline$C(3)-C(4)$ & $1.378(3)$ & $\mathrm{C}(2)-\mathrm{C}(7)-\mathrm{C}(8)$ & $119.25(16)$ \\
\hline $\mathrm{C}(6)-\mathrm{C}(5)$ & $1.369(3)$ & $\mathrm{C}(9)-\mathrm{C}(8)-\mathrm{C}(7)$ & $120.57(17)$ \\
\hline $\mathrm{C}(13)-\mathrm{C}(14)$ & $1.409(3)$ & $\mathrm{C}(10)-\mathrm{C}(1)-\mathrm{C}(2)$ & $118.27(16)$ \\
\hline $\mathrm{C}(13)-\mathrm{C}(12)$ & $1.427(2)$ & $\mathrm{C}(10)-\mathrm{C}(1)-\mathrm{C}(18)$ & $118.77(16)$ \\
\hline $\mathrm{C}(4)-\mathrm{C}(5)$ & $1.387(3)$ & $\mathrm{C}(2)-\mathrm{C}(1)-\mathrm{C}(18)$ & $122.95(15)$ \\
\hline $\mathrm{C}(14)-\mathrm{C}(15)$ & $1.372(3)$ & $\mathrm{C}(8)-\mathrm{C}(9)-\mathrm{C}(10)$ & $121.72(16)$ \\
\hline $\mathrm{C}(16)-\mathrm{C}(15)$ & $1.384(3)$ & $\mathrm{C}(4)-\mathrm{C}(3)-\mathrm{C}(2)$ & $120.98(17)$ \\
\hline \multirow[t]{13}{*}{$\mathrm{C}(11)-\mathrm{C}(12)$} & $1.344(3)$ & $\mathrm{C}(4)-\mathrm{C}(3)-\mathrm{S}(1)$ & $116.33(14)$ \\
\hline & & $\mathrm{C}(2)-\mathrm{C}(3)-\mathrm{S}(1)$ & $122.67(14)$ \\
\hline & & $\mathrm{C}(5)-\mathrm{C}(6)-\mathrm{C}(7)$ & $120.37(18)$ \\
\hline & & $\mathrm{C}(14)-\mathrm{C}(13)-\mathrm{C}(18)$ & $120.41(16)$ \\
\hline & & $\mathrm{C}(14)-\mathrm{C}(13)-\mathrm{C}(12)$ & $120.42(16)$ \\
\hline & & $\mathrm{C}(18)-\mathrm{C}(13)-\mathrm{C}(12)$ & $119.17(16)$ \\
\hline & & $\mathrm{C}(3)-\mathrm{C}(4)-\mathrm{C}(5)$ & $121.01(17)$ \\
\hline & & $C(15)-C(14)-C(13)$ & $120.63(17)$ \\
\hline & & $\mathrm{C}(17)-\mathrm{C}(16)-\mathrm{C}(15)$ & $121.26(17)$ \\
\hline & & $\mathrm{C}(12)-\mathrm{C}(11)-\mathrm{C}(10)$ & $121.62(17)$ \\
\hline & & $C(6)-C(5)-C(4)$ & $120.07(17)$ \\
\hline & & $\mathrm{C}(11)-\mathrm{C}(12)-\mathrm{C}(13)$ & $120.95(17)$ \\
\hline & & $\mathrm{C}(14)-\mathrm{C}(15)-\mathrm{C}(16)$ & $119.83(17)$ \\
\hline
\end{tabular}


Table 4. Anisotropic displacement parameters $\left(\AA^{2} \times 10^{3}\right)$ for naphtho[2,1,8,7-klmn]thioxanthene (2). The anisotropic displacement factor exponent takes the form: $-2 \pi^{2}\left[h^{2} a^{* 2} U^{11}+\ldots+2 h k a^{*} b^{*} U^{12}\right]$

\begin{tabular}{lcccccc}
\hline & $\mathrm{U}^{11}$ & $\mathrm{U}^{22}$ & $\mathrm{U}^{33}$ & $\mathrm{U}^{23}$ & $\mathrm{U}^{13}$ & $\mathrm{U}^{12}$ \\
\hline $\mathrm{S}(1)$ & $37(1)$ & $33(1)$ & $29(1)$ & $8(1)$ & $-5(1)$ & $4(1)$ \\
$\mathrm{C}(18)$ & $20(1)$ & $20(1)$ & $27(1)$ & $-2(1)$ & $-3(1)$ & $-7(1)$ \\
$\mathrm{C}(10)$ & $24(1)$ & $23(1)$ & $26(1)$ & $1(1)$ & $-4(1)$ & $-5(1)$ \\
$\mathrm{C}(2)$ & $21(1)$ & $21(1)$ & $26(1)$ & $-1(1)$ & $-2(1)$ & $-8(1)$ \\
$\mathrm{C}(17)$ & $23(1)$ & $21(1)$ & $31(1)$ & $0(1)$ & $-4(1)$ & $-4(1)$ \\
$\mathrm{C}(7)$ & $24(1)$ & $22(1)$ & $32(1)$ & $-3(1)$ & $-1(1)$ & $-5(1)$ \\
$\mathrm{C}(8)$ & $26(1)$ & $21(1)$ & $42(1)$ & $-4(1)$ & $-3(1)$ & $1(1)$ \\
$\mathrm{C}(1)$ & $21(1)$ & $19(1)$ & $27(1)$ & $0(1)$ & $-2(1)$ & $-4(1)$ \\
$\mathrm{C}(9)$ & $28(1)$ & $23(1)$ & $32(1)$ & $5(1)$ & $-6(1)$ & $0(1)$ \\
$\mathrm{C}(3)$ & $24(1)$ & $26(1)$ & $28(1)$ & $1(1)$ & $-2(1)$ & $-6(1)$ \\
$\mathrm{C}(6)$ & $30(1)$ & $30(1)$ & $39(1)$ & $-9(1)$ & $7(1)$ & $-4(1)$ \\
$\mathrm{C}(13)$ & $23(1)$ & $24(1)$ & $30(1)$ & $-4(1)$ & $-1(1)$ & $-5(1)$ \\
$\mathrm{C}(4)$ & $32(1)$ & $41(1)$ & $25(1)$ & $4(1)$ & $-1(1)$ & $-10(1)$ \\
$\mathrm{C}(14)$ & $29(1)$ & $30(1)$ & $36(1)$ & $-8(1)$ & $2(1)$ & $-4(1)$ \\
$\mathrm{C}(16)$ & $29(1)$ & $23(1)$ & $43(1)$ & $2(1)$ & $-7(1)$ & $0(1)$ \\
$\mathrm{C}(11)$ & $31(1)$ & $31(1)$ & $28(1)$ & $7(1)$ & $-3(1)$ & $-3(1)$ \\
$\mathrm{C}(5)$ & $38(1)$ & $40(1)$ & $26(1)$ & $-6(1)$ & $5(1)$ & $-11(1)$ \\
$\mathrm{C}(12)$ & $32(1)$ & $35(1)$ & $23(1)$ & $0(1)$ & $2(1)$ & $-4(1)$ \\
$\mathrm{C}(15)$ & $29(1)$ & $25(1)$ & $51(1)$ & $-8(1)$ & $-1(1)$ & $2(1)$ \\
& & & & & & \\
\hline
\end{tabular}

Table 5. Hydrogen coordinates ( $\left.\mathrm{x} 10^{4}\right)$ and isotropic displacement parameters $\left(\AA^{2} \mathrm{x} 10^{3}\right)$ for naphtho[2,1,8,7-klmn]thioxanthene (2).

\begin{tabular}{lrrrr}
\hline & $\mathrm{x}$ & $\mathrm{y}$ & $\mathrm{z}$ & $\mathrm{U}(\mathrm{eq})$ \\
\hline $\mathrm{H}(8 \mathrm{~A})$ & 8359 & -3046 & 2554 & 36 \\
$\mathrm{H}(9 \mathrm{~A})$ & 6577 & -2827 & 3808 & 33 \\
$\mathrm{H}(6 \mathrm{~A})$ & 8514 & -2681 & 1112 & 39 \\
$\mathrm{H}(4 \mathrm{~A})$ & 3492 & -811 & 229 & 39 \\
$\mathrm{H}(14 \mathrm{~A})$ & -3198 & 105 & 4602 & 38 \\
$\mathrm{H}(16 \mathrm{~A})$ & -3125 & 920 & 2318 & 38 \\
$\mathrm{H}(11 \mathrm{~A})$ & 3405 & -2072 & 4755 & 36 \\
$\mathrm{H}(5 \mathrm{~A})$ & 6866 & -1891 & 34 & 42 \\
$\mathrm{H}(12 \mathrm{~A})$ & 11 & -1069 & 5034 & 36 \\
$\mathrm{H}(15 \mathrm{~A})$ & -4863 & 1026 & 3621 & 42 \\
& & & & \\
\hline
\end{tabular}


Table 6. Torsion angles $\left[{ }^{\circ}\right]$ for naphtho[2,1,8,7-klmn $]$ thioxanthene (2).

\begin{tabular}{|c|c|}
\hline $\mathrm{C}(13)-\mathrm{C}(18)-\mathrm{C}(17)-\mathrm{C}(16)$ & $-0.5(2)$ \\
\hline $\mathrm{C}(1)-\mathrm{C}(18)-\mathrm{C}(17)-\mathrm{C}(16)$ & 179.41(15) \\
\hline $\mathrm{C}(13)-\mathrm{C}(18)-\mathrm{C}(17)-\mathrm{S}(1)$ & $178.23(12)$ \\
\hline $\mathrm{C}(1)-\mathrm{C}(18)-\mathrm{C}(17)-\mathrm{S}(1)$ & $-1.9(2)$ \\
\hline $\mathrm{C}(3)-\mathrm{S}(1)-\mathrm{C}(17)-\mathrm{C}(16)$ & $-175.35(13)$ \\
\hline C(3)-S(1)-C(17)-C(18) & $5.88(16)$ \\
\hline$C(3)-C(2)-C(7)-C(6)$ & $-0.2(2)$ \\
\hline $\mathrm{C}(1)-\mathrm{C}(2)-\mathrm{C}(7)-\mathrm{C}(6)$ & $179.10(16)$ \\
\hline $\mathrm{C}(3)-\mathrm{C}(2)-\mathrm{C}(7)-\mathrm{C}(8)$ & $179.55(16)$ \\
\hline $\mathrm{C}(1)-\mathrm{C}(2)-\mathrm{C}(7)-\mathrm{C}(8)$ & $-1.2(2)$ \\
\hline$C(6)-C(7)-C(8)-C(9)$ & $-179.07(17)$ \\
\hline $\mathrm{C}(2)-\mathrm{C}(7)-\mathrm{C}(8)-\mathrm{C}(9)$ & $1.2(3)$ \\
\hline $\mathrm{C}(9)-\mathrm{C}(10)-\mathrm{C}(1)-\mathrm{C}(2)$ & $0.9(2)$ \\
\hline $\mathrm{C}(11)-\mathrm{C}(10)-\mathrm{C}(1)-\mathrm{C}(2)$ & $-178.98(15)$ \\
\hline $\mathrm{C}(9)-\mathrm{C}(10)-\mathrm{C}(1)-\mathrm{C}(18)$ & 179.87(14) \\
\hline $\mathrm{C}(11)-\mathrm{C}(10)-\mathrm{C}(1)-\mathrm{C}(18)$ & $0.0(2)$ \\
\hline$C(7)-C(2)-C(1)-C(10)$ & $0.1(2)$ \\
\hline$C(3)-C(2)-C(1)-C(10)$ & $179.34(15)$ \\
\hline$C(7)-C(2)-C(1)-C(18)$ & $-178.77(14)$ \\
\hline $\mathrm{C}(3)-\mathrm{C}(2)-\mathrm{C}(1)-\mathrm{C}(18)$ & $0.4(3)$ \\
\hline $\mathrm{C}(17)-\mathrm{C}(18)-\mathrm{C}(1)-\mathrm{C}(10)$ & $178.98(15)$ \\
\hline $\mathrm{C}(13)-\mathrm{C}(18)-\mathrm{C}(1)-\mathrm{C}(10)$ & $-1.1(2)$ \\
\hline $\mathrm{C}(17)-\mathrm{C}(18)-\mathrm{C}(1)-\mathrm{C}(2)$ & $-2.1(2)$ \\
\hline $\mathrm{C}(13)-\mathrm{C}(18)-\mathrm{C}(1)-\mathrm{C}(2)$ & $177.78(15)$ \\
\hline$C(7)-C(8)-C(9)-C(10)$ & $-0.2(3)$ \\
\hline $\mathrm{C}(1)-\mathrm{C}(10)-\mathrm{C}(9)-\mathrm{C}(8)$ & $-0.9(3)$ \\
\hline $\mathrm{C}(11)-\mathrm{C}(10)-\mathrm{C}(9)-\mathrm{C}(8)$ & $178.96(17)$ \\
\hline$C(7)-C(2)-C(3)-C(4)$ & $2.6(2)$ \\
\hline $\mathrm{C}(1)-\mathrm{C}(2)-\mathrm{C}(3)-\mathrm{C}(4)$ & $-176.64(16)$ \\
\hline $\mathrm{C}(7)-\mathrm{C}(2)-\mathrm{C}(3)-\mathrm{S}(1)$ & $-175.74(12)$ \\
\hline $\mathrm{C}(1)-\mathrm{C}(2)-\mathrm{C}(3)-\mathrm{S}(1)$ & $5.0(2)$ \\
\hline $\mathrm{C}(17)-\mathrm{S}(1)-\mathrm{C}(3)-\mathrm{C}(4)$ & $174.21(13)$ \\
\hline $\mathrm{C}(17)-\mathrm{S}(1)-\mathrm{C}(3)-\mathrm{C}(2)$ & $-7.38(16)$ \\
\hline$C(2)-C(7)-C(6)-C(5)$ & $-1.9(3)$ \\
\hline$C(8)-C(7)-C(6)-C(5)$ & $178.39(17)$ \\
\hline $\mathrm{C}(17)-\mathrm{C}(18)-\mathrm{C}(13)-\mathrm{C}(14)$ & $0.8(2)$ \\
\hline $\mathrm{C}(1)-\mathrm{C}(18)-\mathrm{C}(13)-\mathrm{C}(14)$ & $-179.09(15)$ \\
\hline $\mathrm{C}(17)-\mathrm{C}(18)-\mathrm{C}(13)-\mathrm{C}(12)$ & $-178.74(15)$ \\
\hline $\mathrm{C}(1)-\mathrm{C}(18)-\mathrm{C}(13)-\mathrm{C}(12)$ & $1.4(2)$ \\
\hline$C(2)-C(3)-C(4)-C(5)$ & $-3.0(3)$ \\
\hline $\mathrm{S}(1)-\mathrm{C}(3)-\mathrm{C}(4)-\mathrm{C}(5)$ & $175.39(14)$ \\
\hline$C(18)-C(13)-C(14)-C(15)$ & $-0.8(3)$ \\
\hline $\mathrm{C}(12)-\mathrm{C}(13)-\mathrm{C}(14)-\mathrm{C}(15)$ & 178.71(17) \\
\hline$C(18)-C(17)-C(16)-C(15)$ & $0.2(3)$ \\
\hline$S(1)-C(17)-C(16)-C(15)$ & $-178.63(14)$ \\
\hline$C(1)-C(10)-C(11)-C(12)$ & $1.0(3)$ \\
\hline $\mathrm{C}(9)-\mathrm{C}(10)-\mathrm{C}(11)-\mathrm{C}(12)$ & $-178.91(17)$ \\
\hline$C(7)-C(6)-C(5)-C(4)$ & $1.6(3)$ \\
\hline$C(3)-C(4)-C(5)-C(6)$ & $0.9(3)$ \\
\hline $\mathrm{C}(10)-\mathrm{C}(11)-\mathrm{C}(12)-\mathrm{C}(13)$ & $-0.7(3)$ \\
\hline $\mathrm{C}(14)-\mathrm{C}(13)-\mathrm{C}(12)-\mathrm{C}(11)$ & $-179.98(17)$ \\
\hline $\mathrm{C}(18)-\mathrm{C}(13)-\mathrm{C}(12)-\mathrm{C}(11)$ & $-0.4(3)$ \\
\hline$C(13)-C(14)-C(15)-C(16)$ & $0.5(3)$ \\
\hline $\mathrm{C}(17)-\mathrm{C}(16)-\mathrm{C}(15)-\mathrm{C}(14)$ & $-0.2(3)$ \\
\hline
\end{tabular}


X-Ray Data for naphtho[2,1,8,7-klmn]xanthene (14):
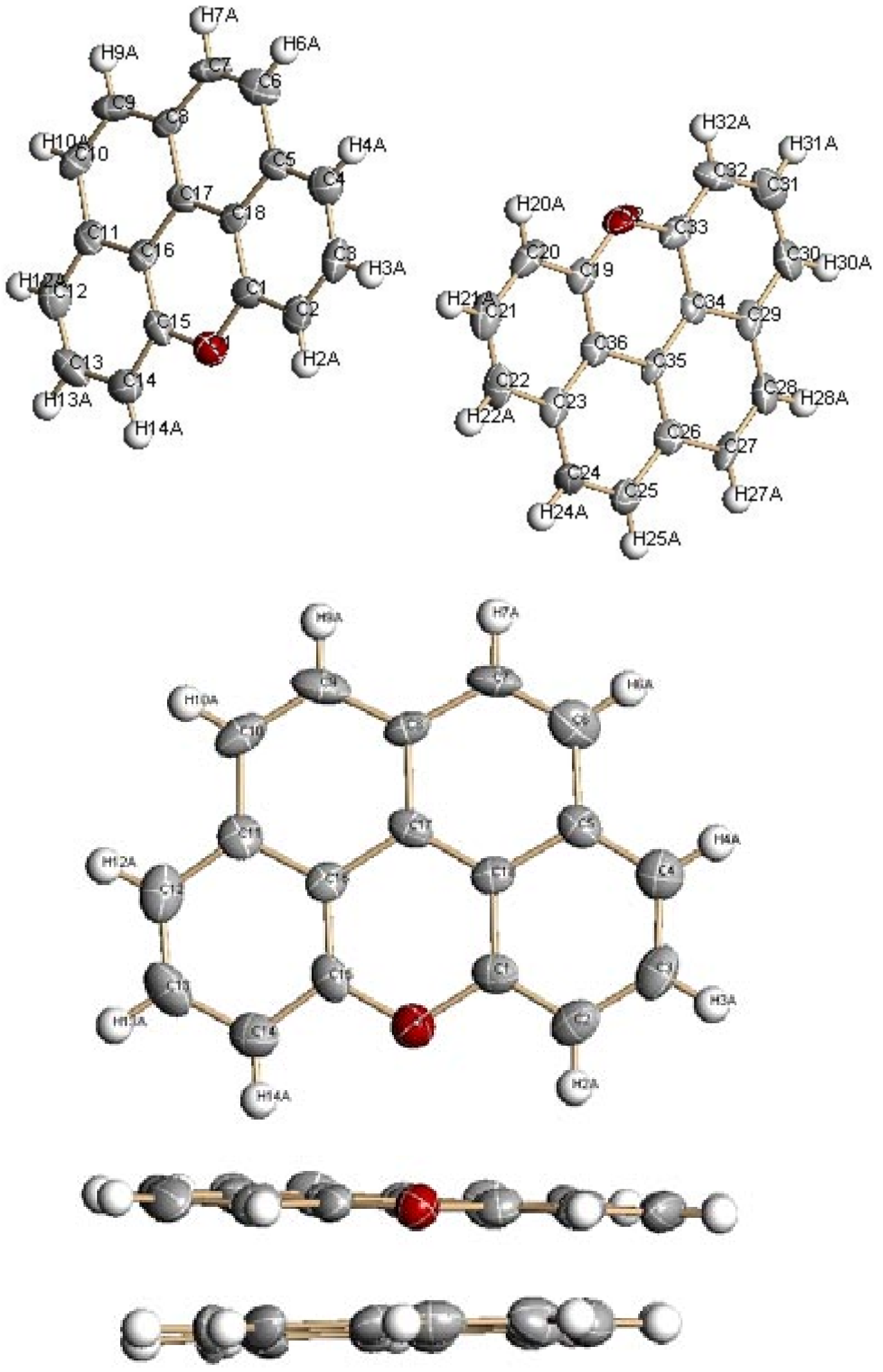

page $\mathrm{S} 20$ 

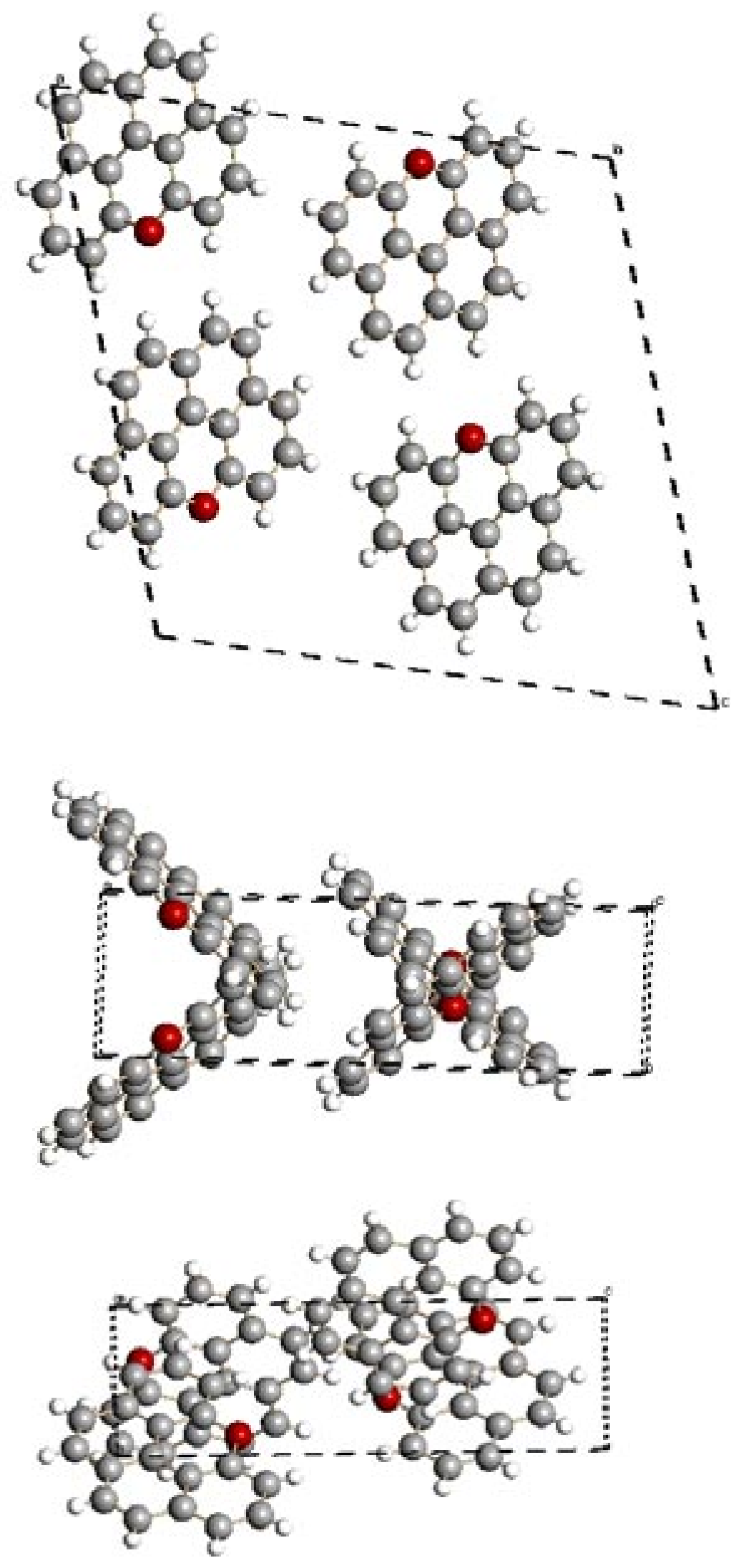
Table 1. Crystal data and structure refinement for naphtho[2,1,8,7-klmn]xanthene (14).

Identification code

Empirical formula

Formula weight

Temperature

Wavelength

Crystal system

Space group

Unit cell dimensions

Volume

Z

Density (calculated)

Absorption coefficient

$\mathrm{F}(000)$

Crystal size

Theta range for data collection

Index ranges

Reflections collected

Independent reflections

Completeness to theta $=28.32^{\circ}$

Absorption correction

Refinement method

Data / restraints / parameters

Goodness-of-fit on $\mathrm{F}^{2}$

Final R indices [I $>2 \operatorname{sigma}(\mathrm{I})]$

$\mathrm{R}$ indices (all data)

Absolute structure parameter

Largest diff. peak and hole pat04b

C18 H10 O

242.26

193(2) K

$0.71073 \AA$

Monoclinic

$\mathrm{Pc}$

$\mathrm{a}=16.087(2) \AA$

$\mathrm{b}=4.6626(7) \AA$

$\mathrm{c}=15.988(2) \AA$

1138.9(3) $\AA^{3}$

4

$1.413 \mathrm{Mg} / \mathrm{m}^{3}$

$0.086 \mathrm{~mm}^{-1}$

504

$0.58 \times 0.20 \times 0.18 \mathrm{~mm}^{3}$

1.33 to $28.32^{\circ}$.

$-21<=\mathrm{h}<=21,-6<=\mathrm{k}<=5,-16<=1<=21$

7912

$4207[\mathrm{R}(\mathrm{int})=0.0622]$

$99.8 \%$

None

Full-matrix least-squares on $\mathrm{F}^{2}$

4207 / 2 / 343

0.889

$\mathrm{R} 1=0.0513, \mathrm{wR} 2=0.1172$

$\mathrm{R} 1=0.0951, \mathrm{wR} 2=0.1363$

10(4)

0.264 and -0.203 e. $\AA^{-3}$

Table 2. Atomic coordinates $\left(\mathrm{x} 10^{4}\right)$ and equivalent isotropic displacement parameters $\left(\AA^{2} \times 10^{3}\right)$ for naphtho[2,1,8,7-klmn]xanthene (14). $\mathrm{U}(\mathrm{eq})$ is defined as one third of the trace of the orthogonalized $\mathrm{U}^{\mathrm{ij}}$ tensor.

\begin{tabular}{|c|c|c|c|c|}
\hline & $\mathrm{x}$ & $\mathrm{y}$ & $\mathrm{Z}$ & $\mathrm{U}(\mathrm{eq})$ \\
\hline $\bar{C}(18)$ & $8054(3)$ & $8043(14)$ & $896(4)$ & $32(1)$ \\
\hline$C(16)$ & $9247(3)$ & $11418(14)$ & $1448(4)$ & $33(1)$ \\
\hline$C(17)$ & $8611(3)$ & $10053(14)$ & $723(3)$ & $34(1)$ \\
\hline $\mathrm{O}(1)$ & $8723(2)$ & $8719(11)$ & $2481(3)$ & $44(1)$ \\
\hline $\mathrm{C}(8)$ & $8559(3)$ & $10814(15)$ & $-159(4)$ & $34(1)$ \\
\hline $\mathrm{C}(11)$ & $9836(3)$ & 13461(15) & $1304(4)$ & $39(2)$ \\
\hline$C(5)$ & $7397(3)$ & $6656(14)$ & $204(4)$ & $38(1)$ \\
\hline$C(2)$ & $7574(4)$ & $5373(16)$ & $1964(4)$ & $45(2)$ \\
\hline$C(15)$ & $9278(3)$ & $10722(14)$ & $2295(4)$ & $39(2)$ \\
\hline$C(1)$ & $8107(3)$ & $7338(14)$ & $1765(4)$ & $39(2)$ \\
\hline $\mathrm{C}(10)$ & $9756(4)$ & $14154(16)$ & $393(4)$ & $45(2)$ \\
\hline $\mathrm{C}(9)$ & $9156(4)$ & $12805(14)$ & $-279(4)$ & $49(2)$ \\
\hline$C(12)$ & $10425(4)$ & $14765(18)$ & $2042(4)$ & $52(2)$ \\
\hline $\mathrm{C}(6)$ & $7360(4)$ & $7455(14)$ & $-694(4)$ & $48(2)$ \\
\hline$C(14)$ & $9848(4)$ & $11988(17)$ & $3029(4)$ & $47(2)$ \\
\hline$C(7)$ & $7896(4)$ & $9410(17)$ & $-840(3)$ & $45(2)$ \\
\hline C(4) & $6860(4)$ & $4646(15)$ & $395(4)$ & $47(2)$ \\
\hline$C(3)$ & $6952(4)$ & 4041(16) & $1272(4)$ & $48(2)$ \\
\hline$C(13)$ & $10438(4)$ & $14018(18)$ & $2877(4)$ & $53(2)$ \\
\hline $\mathrm{C}(34)$ & $3012(3)$ & $6405(15)$ & $1569(4)$ & $33(1)$ \\
\hline$C(36)$ & $4226(3)$ & $3042(14)$ & 2111(4) & $32(1)$ \\
\hline
\end{tabular}




\begin{tabular}{lrrrr}
$\mathrm{C}(35)$ & $3628(3)$ & $5108(13)$ & $2294(3)$ & $29(1)$ \\
$\mathrm{O}(2)$ & $3550(2)$ & $3682(11)$ & $554(3)$ & $42(1)$ \\
$\mathrm{C}(33)$ & $2976(4)$ & $5726(14)$ & $682(4)$ & $38(1)$ \\
$\mathrm{C}(29)$ & $2435(3)$ & $8502(14)$ & $1698(4)$ & $38(1)$ \\
$\mathrm{C}(19)$ & $4157(3)$ & $2394(13)$ & $1245(4)$ & $34(1)$ \\
$\mathrm{C}(23)$ & $4851(3)$ & $1680(14)$ & $2820(4)$ & $38(1)$ \\
$\mathrm{C}(26)$ & $3674(3)$ & $5770(15)$ & $3135(4)$ & $37(1)$ \\
$\mathrm{C}(32)$ & $2389(4)$ & $7046(15)$ & $-4(4)$ & $46(2)$ \\
$\mathrm{C}(20)$ & $4697(4)$ & $372(16)$ & $1058(4)$ & $44(2)$ \\
$\mathrm{C}(22)$ & $5407(4)$ & $-387(15)$ & $2593(5)$ & $46(2)$ \\
$\mathrm{C}(28)$ & $2484(3)$ & $9130(15)$ & $2574(4)$ & $43(2)$ \\
$\mathrm{C}(27)$ & $3082(3)$ & $7897(12)$ & $3279(4)$ & $36(1)$ \\
$\mathrm{C}(30)$ & $1832(3)$ & $9801(17)$ & $963(4)$ & $46(2)$ \\
$\mathrm{C}(25)$ & $4332(3)$ & $4443(16)$ & $3864(4)$ & $40(1)$ \\
$\mathrm{C}(31)$ & $1831(4)$ & $9126(18)$ & $131(5)$ & $52(2)$ \\
$\mathrm{C}(24)$ & $4899(3)$ & $2441(16)$ & $3678(4)$ & $45(2)$ \\
$\mathrm{C}(21)$ & $5328(4)$ & $-1018(16)$ & $1760(5)$ & $49(2)$ \\
& & & & \\
\hline
\end{tabular}

Table 3. Bond lengths $[\AA]$ and angles $\left[{ }^{\circ}\right]$ for naphtho[2,1,8,7-klmn $]$ xanthene (14).

\begin{tabular}{|c|c|c|c|}
\hline $\mathrm{C}(18)-\mathrm{C}(17)$ & $1.383(8)$ & $\mathrm{C}(34)-\mathrm{C}(35)$ & $1.405(7)$ \\
\hline $\mathrm{C}(18)-\mathrm{C}(1)$ & $1.404(8)$ & $C(34)-C(29)$ & $1.406(8)$ \\
\hline $\mathrm{C}(18)-\mathrm{C}(5)$ & $1.423(8)$ & $\mathrm{C}(34)-\mathrm{C}(33)$ & $1.437(8)$ \\
\hline$C(16)-C(15)$ & $1.379(8)$ & $C(36)-C(19)$ & $1.387(9)$ \\
\hline $\mathrm{C}(16)-\mathrm{C}(11)$ & $1.413(9)$ & $\mathrm{C}(36)-\mathrm{C}(23)$ & $1.409(8)$ \\
\hline $\mathrm{C}(16)-\mathrm{C}(17)$ & $1.432(8)$ & $\mathrm{C}(36)-\mathrm{C}(35)$ & $1.455(7)$ \\
\hline $\mathrm{C}(17)-\mathrm{C}(8)$ & $1.432(8)$ & $\mathrm{C}(35)-\mathrm{C}(26)$ & $1.357(8)$ \\
\hline $\mathrm{O}(1)-\mathrm{C}(15)$ & $1.387(7)$ & $\mathrm{O}(2)-\mathrm{C}(19)$ & $1.363(7)$ \\
\hline $\mathrm{O}(1)-\mathrm{C}(1)$ & $1.412(7)$ & $\mathrm{O}(2)-\mathrm{C}(33)$ & $1.387(7)$ \\
\hline $\mathrm{C}(8)-\mathrm{C}(9)$ & 1.391(9) & $\mathrm{C}(33)-\mathrm{C}(32)$ & $1.351(8)$ \\
\hline $\mathrm{C}(8)-\mathrm{C}(7)$ & $1.422(8)$ & $\mathrm{C}(29)-\mathrm{C}(30)$ & $1.406(8)$ \\
\hline$C(11)-C(12)$ & $1.399(8)$ & $\mathrm{C}(29)-\mathrm{C}(28)$ & $1.410(9)$ \\
\hline $\mathrm{C}(11)-\mathrm{C}(10)$ & $1.458(9)$ & $\mathrm{C}(19)-\mathrm{C}(20)$ & $1.376(8)$ \\
\hline$C(5)-C(4)$ & $1.372(9)$ & $\mathrm{C}(23)-\mathrm{C}(24)$ & $1.396(9)$ \\
\hline$C(5)-C(6)$ & $1.466(9)$ & $\mathrm{C}(23)-\mathrm{C}(22)$ & $1.437(8)$ \\
\hline $\mathrm{C}(2)-\mathrm{C}(1)$ & $1.360(9)$ & $\mathrm{C}(26)-\mathrm{C}(27)$ & $1.443(8)$ \\
\hline $\mathrm{C}(2)-\mathrm{C}(3)$ & $1.385(9)$ & $\mathrm{C}(26)-\mathrm{C}(25)$ & $1.445(8)$ \\
\hline$C(15)-C(14)$ & $1.376(8)$ & $\mathrm{C}(32)-\mathrm{C}(31)$ & $1.383(10)$ \\
\hline$C(10)-C(9)$ & $1.354(9)$ & $\mathrm{C}(20)-\mathrm{C}(21)$ & 1.413(9) \\
\hline $\mathrm{C}(12)-\mathrm{C}(13)$ & $1.373(10)$ & $\mathrm{C}(22)-\mathrm{C}(21)$ & $1.329(10)$ \\
\hline$C(6)-C(7)$ & $1.326(9)$ & $\mathrm{C}(28)-\mathrm{C}(27)$ & $1.359(8)$ \\
\hline$C(14)-C(13)$ & $1.414(11)$ & $\mathrm{C}(30)-\mathrm{C}(31)$ & $1.365(9)$ \\
\hline C(4)-C(3) & $1.392(10)$ & $C(25)-C(24)$ & $1.400(9)$ \\
\hline
\end{tabular}




\begin{tabular}{|c|c|c|c|}
\hline $\mathrm{C}(17)-\mathrm{C}(18)-\mathrm{C}(1)$ & $120.9(5)$ & $\mathrm{C}(35)-\mathrm{C}(34)-\mathrm{C}(29)$ & $120.3(5)$ \\
\hline $\mathrm{C}(17)-\mathrm{C}(18)-\mathrm{C}(5)$ & $121.5(5)$ & $\mathrm{C}(35)-\mathrm{C}(34)-\mathrm{C}(33)$ & $121.3(5)$ \\
\hline $\mathrm{C}(1)-\mathrm{C}(18)-\mathrm{C}(5)$ & $117.6(6)$ & $\mathrm{C}(29)-\mathrm{C}(34)-\mathrm{C}(33)$ & $118.3(5)$ \\
\hline$C(15)-C(16)-C(11)$ & $119.9(5)$ & $\mathrm{C}(19)-\mathrm{C}(36)-\mathrm{C}(23)$ & $121.2(5)$ \\
\hline $\mathrm{C}(15)-\mathrm{C}(16)-\mathrm{C}(17)$ & $119.1(5)$ & $\mathrm{C}(19)-\mathrm{C}(36)-\mathrm{C}(35)$ & $119.6(5)$ \\
\hline $\mathrm{C}(11)-\mathrm{C}(16)-\mathrm{C}(17)$ & $120.9(5)$ & $C(23)-C(36)-C(35)$ & $119.1(5)$ \\
\hline $\mathrm{C}(18)-\mathrm{C}(17)-\mathrm{C}(8)$ & $121.6(5)$ & $\mathrm{C}(26)-\mathrm{C}(35)-\mathrm{C}(34)$ & $121.7(5)$ \\
\hline $\mathrm{C}(18)-\mathrm{C}(17)-\mathrm{C}(16)$ & $118.9(5)$ & $\mathrm{C}(26)-\mathrm{C}(35)-\mathrm{C}(36)$ & $121.0(5)$ \\
\hline$C(8)-C(17)-C(16)$ & $119.5(5)$ & $\mathrm{C}(34)-\mathrm{C}(35)-\mathrm{C}(36)$ & $117.3(5)$ \\
\hline $\mathrm{C}(15)-\mathrm{O}(1)-\mathrm{C}(1)$ & $117.9(4)$ & $\mathrm{C}(19)-\mathrm{O}(2)-\mathrm{C}(33)$ & $121.6(4)$ \\
\hline $\mathrm{C}(9)-\mathrm{C}(8)-\mathrm{C}(7)$ & $125.9(5)$ & $\mathrm{C}(32)-\mathrm{C}(33)-\mathrm{O}(2)$ & $121.5(5)$ \\
\hline $\mathrm{C}(9)-\mathrm{C}(8)-\mathrm{C}(17)$ & $118.1(5)$ & $\mathrm{C}(32)-\mathrm{C}(33)-\mathrm{C}(34)$ & $120.1(6)$ \\
\hline $\mathrm{C}(7)-\mathrm{C}(8)-\mathrm{C}(17)$ & $116.0(5)$ & $\mathrm{O}(2)-\mathrm{C}(33)-\mathrm{C}(34)$ & $118.3(5)$ \\
\hline $\mathrm{C}(12)-\mathrm{C}(11)-\mathrm{C}(16)$ & $117.9(5)$ & $\mathrm{C}(30)-\mathrm{C}(29)-\mathrm{C}(34)$ & $119.4(5)$ \\
\hline $\mathrm{C}(12)-\mathrm{C}(11)-\mathrm{C}(10)$ & $124.8(6)$ & $\mathrm{C}(30)-\mathrm{C}(29)-\mathrm{C}(28)$ & $123.4(6)$ \\
\hline $\mathrm{C}(16)-\mathrm{C}(11)-\mathrm{C}(10)$ & $117.3(5)$ & $\mathrm{C}(34)-\mathrm{C}(29)-\mathrm{C}(28)$ & $117.2(5)$ \\
\hline $\mathrm{C}(4)-\mathrm{C}(5)-\mathrm{C}(18)$ & $120.3(6)$ & $\mathrm{O}(2)-\mathrm{C}(19)-\mathrm{C}(20)$ & $117.8(5)$ \\
\hline$C(4)-C(5)-C(6)$ & $123.8(6)$ & $\mathrm{O}(2)-\mathrm{C}(19)-\mathrm{C}(36)$ & $121.7(5)$ \\
\hline$C(18)-C(5)-C(6)$ & $115.9(6)$ & $\mathrm{C}(20)-\mathrm{C}(19)-\mathrm{C}(36)$ & $120.5(6)$ \\
\hline$C(1)-C(2)-C(3)$ & $117.8(6)$ & $\mathrm{C}(24)-\mathrm{C}(23)-\mathrm{C}(36)$ & $118.8(5)$ \\
\hline $\mathrm{C}(14)-\mathrm{C}(15)-\mathrm{C}(16)$ & $123.1(6)$ & $\mathrm{C}(24)-\mathrm{C}(23)-\mathrm{C}(22)$ & $124.9(6)$ \\
\hline $\mathrm{C}(14)-\mathrm{C}(15)-\mathrm{O}(1)$ & $114.1(5)$ & $C(36)-C(23)-C(22)$ & $116.3(5)$ \\
\hline $\mathrm{C}(16)-\mathrm{C}(15)-\mathrm{O}(1)$ & $122.8(5)$ & $\mathrm{C}(35)-\mathrm{C}(26)-\mathrm{C}(27)$ & $118.7(5)$ \\
\hline$C(2)-C(1)-C(18)$ & $122.8(6)$ & $C(35)-C(26)-C(25)$ & $120.0(5)$ \\
\hline $\mathrm{C}(2)-\mathrm{C}(1)-\mathrm{O}(1)$ & $116.8(5)$ & $\mathrm{C}(27)-\mathrm{C}(26)-\mathrm{C}(25)$ & $121.2(5)$ \\
\hline $\mathrm{C}(18)-\mathrm{C}(1)-\mathrm{O}(1)$ & $120.4(5)$ & $\mathrm{C}(33)-\mathrm{C}(32)-\mathrm{C}(31)$ & $121.0(6)$ \\
\hline $\mathrm{C}(9)-\mathrm{C}(10)-\mathrm{C}(11)$ & $120.5(6)$ & $\mathrm{C}(19)-\mathrm{C}(20)-\mathrm{C}(21)$ & $119.2(6)$ \\
\hline $\mathrm{C}(10)-\mathrm{C}(9)-\mathrm{C}(8)$ & $123.5(5)$ & $C(21)-C(22)-C(23)$ & $122.0(6)$ \\
\hline $\mathrm{C}(13)-\mathrm{C}(12)-\mathrm{C}(11)$ & $120.6(7)$ & $\mathrm{C}(27)-\mathrm{C}(28)-\mathrm{C}(29)$ & $122.7(5)$ \\
\hline$C(7)-C(6)-C(5)$ & $121.3(6)$ & $\mathrm{C}(28)-\mathrm{C}(27)-\mathrm{C}(26)$ & $119.3(5)$ \\
\hline$C(15)-C(14)-C(13)$ & $116.4(6)$ & $\mathrm{C}(31)-\mathrm{C}(30)-\mathrm{C}(29)$ & $120.3(6)$ \\
\hline$C(6)-C(7)-C(8)$ & $123.6(5)$ & $C(24)-C(25)-C(26)$ & $118.4(5)$ \\
\hline $\mathrm{C}(5)-\mathrm{C}(4)-\mathrm{C}(3)$ & 119.1(6) & $\mathrm{C}(30)-\mathrm{C}(31)-\mathrm{C}(32)$ & $120.7(6)$ \\
\hline$C(2)-C(3)-C(4)$ & $122.4(6)$ & $C(23)-C(24)-C(25)$ & $122.6(5)$ \\
\hline $\mathrm{C}(12)-\mathrm{C}(13)-\mathrm{C}(14)$ & $122.0(6)$ & $\mathrm{C}(22)-\mathrm{C}(21)-\mathrm{C}(20)$ & $120.8(6)$ \\
\hline
\end{tabular}

Table 4. Anisotropic displacement parameters $\left(\AA^{2} \times 10^{3}\right)$ for naphtho[2,1,8,7-klmn]xanthene (14). The anisotropic displacement factor exponent takes the form: $-2 \pi^{2}\left[h^{2} a^{* 2} U^{11}+\ldots+2 h k a^{*} b^{*} U^{12}\right]$

\begin{tabular}{lcccccc}
\hline & $\mathrm{U}^{11}$ & $\mathrm{U}^{22}$ & $\mathrm{U}^{33}$ & $\mathrm{U}^{23}$ & $\mathrm{U}^{13}$ & $\mathrm{U}^{12}$ \\
\hline $\mathrm{C}(18)$ & $33(3)$ & $32(3)$ & $35(3)$ & $2(3)$ & $16(2)$ & $12(3)$ \\
$\mathrm{C}(16)$ & $36(3)$ & $29(3)$ & $39(3)$ & $-2(2)$ & $18(2)$ & $6(3)$ \\
$\mathrm{C}(17)$ & $34(3)$ & $37(4)$ & $29(3)$ & $-1(2)$ & $5(2)$ & $13(3)$ \\
$\mathrm{O}(1)$ & $40(2)$ & $46(3)$ & $43(2)$ & $2(2)$ & $11(2)$ & $1(2)$ \\
$\mathrm{C}(8)$ & $39(3)$ & $35(4)$ & $33(3)$ & $11(3)$ & $19(2)$ & $10(3)$ \\
$\mathrm{C}(11)$ & $39(3)$ & $37(4)$ & $45(3)$ & $-7(3)$ & $17(3)$ & $8(3)$ \\
$\mathrm{C}(5)$ & $36(3)$ & $39(4)$ & $37(3)$ & $-4(3)$ & $9(2)$ & $8(3)$ \\
$\mathrm{C}(2)$ & $38(3)$ & $47(4)$ & $53(4)$ & $12(3)$ & $21(3)$ & $11(3)$ \\
$\mathrm{C}(15)$ & $27(2)$ & $41(4)$ & $42(3)$ & $-6(3)$ & $3(2)$ & $4(3)$ \\
$\mathrm{C}(1)$ & $37(3)$ & $43(4)$ & $35(3)$ & $5(3)$ & $10(2)$ & $11(3)$ \\
$\mathrm{C}(10)$ & $57(3)$ & $35(4)$ & $55(4)$ & $9(3)$ & $37(3)$ & $6(3)$ \\
$\mathrm{C}(9)$ & $70(4)$ & $50(4)$ & $26(3)$ & $1(3)$ & $15(3)$ & $16(3)$ \\
$\mathrm{C}(12)$ & $43(3)$ & $38(4)$ & $77(5)$ & $-6(4)$ & $20(4)$ & $1(3)$
\end{tabular}




\begin{tabular}{llllcrr}
$\mathrm{C}(6)$ & $57(3)$ & $31(3)$ & $49(4)$ & $6(3)$ & $8(3)$ & $12(3)$ \\
$\mathrm{C}(14)$ & $43(3)$ & $54(5)$ & $43(3)$ & $2(3)$ & $13(3)$ & $9(3)$ \\
$\mathrm{C}(7)$ & $65(4)$ & $49(4)$ & $23(3)$ & $5(3)$ & $15(3)$ & $8(3)$ \\
$\mathrm{C}(4)$ & $42(3)$ & $42(4)$ & $55(4)$ & $4(3)$ & $13(3)$ & $10(3)$ \\
$\mathrm{C}(3)$ & $36(3)$ & $43(4)$ & $73(5)$ & $1(3)$ & $28(3)$ & $2(3)$ \\
$\mathrm{C}(13)$ & $42(3)$ & $54(5)$ & $50(4)$ & $-5(3)$ & $-6(3)$ & $8(3)$ \\
$\mathrm{C}(34)$ & $26(2)$ & $32(3)$ & $40(3)$ & $-8(3)$ & $9(2)$ & $-12(2)$ \\
$\mathrm{C}(36)$ & $31(2)$ & $24(3)$ & $42(3)$ & $-5(3)$ & $14(2)$ & $-5(3)$ \\
$\mathrm{C}(35)$ & $30(2)$ & $20(3)$ & $46(3)$ & $-7(2)$ & $23(2)$ & $-5(2)$ \\
$\mathrm{O}(2)$ & $52(2)$ & $48(3)$ & $32(2)$ & $-12(2)$ & $21(2)$ & $-4(2)$ \\
$\mathrm{C}(33)$ & $46(3)$ & $32(4)$ & $42(3)$ & $-13(3)$ & $24(3)$ & $-13(3)$ \\
$\mathrm{C}(29)$ & $28(2)$ & $29(3)$ & $59(4)$ & $-11(3)$ & $16(3)$ & $-5(2)$ \\
$\mathrm{C}(19)$ & $33(3)$ & $27(3)$ & $47(4)$ & $-2(3)$ & $18(3)$ & $-7(3)$ \\
$\mathrm{C}(23)$ & $34(3)$ & $26(3)$ & $58(4)$ & $-4(3)$ & $20(3)$ & $-6(3)$ \\
$\mathrm{C}(26)$ & $36(3)$ & $32(4)$ & $46(3)$ & $3(3)$ & $16(2)$ & $-4(3)$ \\
$\mathrm{C}(32)$ & $56(4)$ & $43(4)$ & $31(3)$ & $2(3)$ & $1(3)$ & $-11(3)$ \\
$\mathrm{C}(20)$ & $55(3)$ & $36(4)$ & $54(4)$ & $-11(3)$ & $37(3)$ & $-9(3)$ \\
$\mathrm{C}(22)$ & $36(3)$ & $36(4)$ & $72(5)$ & $12(3)$ & $23(3)$ & $9(3)$ \\
$\mathrm{C}(28)$ & $28(2)$ & $41(4)$ & $62(4)$ & $-9(3)$ & $15(2)$ & $-2(3)$ \\
$\mathrm{C}(27)$ & $33(2)$ & $30(3)$ & $56(3)$ & $-14(3)$ & $28(2)$ & $-3(2)$ \\
$\mathrm{C}(30)$ & $29(3)$ & $44(4)$ & $60(4)$ & $-3(3)$ & $8(3)$ & $-1(3)$ \\
$\mathrm{C}(25)$ & $35(3)$ & $44(4)$ & $45(3)$ & $1(3)$ & $16(2)$ & $-5(3)$ \\
$\mathrm{C}(31)$ & $43(3)$ & $47(4)$ & $61(4)$ & $12(3)$ & $8(3)$ & $0(3)$ \\
$\mathrm{C}(24)$ & $34(3)$ & $66(4)$ & $36(3)$ & $17(3)$ & $12(3)$ & $6(3)$ \\
$\mathrm{C}(21)$ & $42(3)$ & $30(4)$ & $84(5)$ & $-11(3)$ & $31(3)$ & $-2(3)$ \\
& & & & & & \\
\hline
\end{tabular}

Table 5. Hydrogen coordinates ( x $\left.10^{4}\right)$ and isotropic displacement parameters $\left(\AA^{2} \times 10^{3}\right)$ for naphtho[2,1,8,7-klmn]xanthene (14).

\begin{tabular}{lrrrr}
\hline & $\mathrm{x}$ & $\mathrm{y}$ & $\mathrm{z}$ & $\mathrm{U}(\mathrm{eq})$ \\
\hline $\mathrm{H}(2 \mathrm{~A})$ & & & & \\
$\mathrm{H}(10 \mathrm{~A})$ & 7624 & 4939 & 2546 & 53 \\
$\mathrm{H}(9 \mathrm{~A})$ & 10121 & 15532 & 273 & 54 \\
$\mathrm{H}(12 \mathrm{~A})$ & 9141 & 13232 & -851 & 59 \\
$\mathrm{H}(6 \mathrm{~A})$ & 10811 & 16151 & 1967 & 63 \\
$\mathrm{H}(14 \mathrm{~A})$ & 6952 & 6574 & -1169 & 57 \\
$\mathrm{H}(7 \mathrm{~A})$ & 9846 & 11534 & 3595 & 57 \\
$\mathrm{H}(4 \mathrm{~A})$ & 7836 & 9889 & -1421 & 54 \\
$\mathrm{H}(3 \mathrm{~A})$ & 6440 & 3700 & -56 & 56 \\
$\mathrm{H}(13 \mathrm{~A})$ & 6582 & 2691 & 1398 & 58 \\
$\mathrm{H}(32 \mathrm{~A})$ & 10848 & 14873 & 3357 & 64 \\
$\mathrm{H}(20 \mathrm{~A})$ & 2359 & 6548 & -576 & 55 \\
$\mathrm{H}(22 \mathrm{~A})$ & 4646 & -76 & 478 & 52 \\
$\mathrm{H}(28 \mathrm{~A})$ & 5835 & -1313 & 3041 & 55 \\
$\mathrm{H}(27 \mathrm{~A})$ & 2089 & 10441 & 2674 & 52 \\
$\mathrm{H}(30 \mathrm{~A})$ & 3110 & 8420 & 3849 & 43 \\
$\mathrm{H}(25 \mathrm{~A})$ & 1431 & 11125 & 1043 & 55 \\
$\mathrm{H}(31 \mathrm{~A})$ & 4378 & 4907 & 4442 & 48 \\
$\mathrm{H}(24 \mathrm{~A})$ & 1452 & 10075 & -349 & 63 \\
$\mathrm{H}(21 \mathrm{~A})$ & 5324 & 1585 & 4145 & 54 \\
& 5694 & -2390 & 1639 & 59 \\
\hline
\end{tabular}


Table 6. Torsion angles $\left[{ }^{\circ}\right]$ for naphtho[2,1,8,7-klmn $]$ xanthene (14).

\begin{tabular}{|c|c|}
\hline $\mathrm{C}(1)-\mathrm{C}(18)-\mathrm{C}(17)-\mathrm{C}(8)$ & $-179.4(6)$ \\
\hline $\mathrm{C}(5)-\mathrm{C}(18)-\mathrm{C}(17)-\mathrm{C}(8)$ & $-0.7(8)$ \\
\hline $\mathrm{C}(1)-\mathrm{C}(18)-\mathrm{C}(17)-\mathrm{C}(16)$ & $0.0(7)$ \\
\hline $\mathrm{C}(5)-\mathrm{C}(18)-\mathrm{C}(17)-\mathrm{C}(16)$ & $178.7(5)$ \\
\hline $\mathrm{C}(15)-\mathrm{C}(16)-\mathrm{C}(17)-\mathrm{C}(18)$ & $-0.9(7)$ \\
\hline $\mathrm{C}(11)-\mathrm{C}(16)-\mathrm{C}(17)-\mathrm{C}(18)$ & $179.3(5)$ \\
\hline $\mathrm{C}(15)-\mathrm{C}(16)-\mathrm{C}(17)-\mathrm{C}(8)$ & $178.5(6)$ \\
\hline $\mathrm{C}(11)-\mathrm{C}(16)-\mathrm{C}(17)-\mathrm{C}(8)$ & $-1.3(8)$ \\
\hline $\mathrm{C}(18)-\mathrm{C}(17)-\mathrm{C}(8)-\mathrm{C}(9)$ & $-178.5(5)$ \\
\hline$C(16)-C(17)-C(8)-C(9)$ & $2.1(8)$ \\
\hline $\mathrm{C}(18)-\mathrm{C}(17)-\mathrm{C}(8)-\mathrm{C}(7)$ & $0.8(8)$ \\
\hline $\mathrm{C}(16)-\mathrm{C}(17)-\mathrm{C}(8)-\mathrm{C}(7)$ & $-178.6(5)$ \\
\hline $\mathrm{C}(15)-\mathrm{C}(16)-\mathrm{C}(11)-\mathrm{C}(12)$ & $-1.4(8)$ \\
\hline $\mathrm{C}(17)-\mathrm{C}(16)-\mathrm{C}(11)-\mathrm{C}(12)$ & $178.4(5)$ \\
\hline$C(15)-C(16)-C(11)-C(10)$ & $-178.6(6)$ \\
\hline $\mathrm{C}(17)-\mathrm{C}(16)-\mathrm{C}(11)-\mathrm{C}(10)$ & $1.2(8)$ \\
\hline $\mathrm{C}(17)-\mathrm{C}(18)-\mathrm{C}(5)-\mathrm{C}(4)$ & $179.2(5)$ \\
\hline $\mathrm{C}(1)-\mathrm{C}(18)-\mathrm{C}(5)-\mathrm{C}(4)$ & $-2.0(8)$ \\
\hline $\mathrm{C}(17)-\mathrm{C}(18)-\mathrm{C}(5)-\mathrm{C}(6)$ & $1.0(7)$ \\
\hline $\mathrm{C}(1)-\mathrm{C}(18)-\mathrm{C}(5)-\mathrm{C}(6)$ & $179.8(5)$ \\
\hline $\mathrm{C}(11)-\mathrm{C}(16)-\mathrm{C}(15)-\mathrm{C}(14)$ & $1.8(9)$ \\
\hline$C(17)-C(16)-C(15)-C(14)$ & $-178.0(5)$ \\
\hline $\mathrm{C}(11)-\mathrm{C}(16)-\mathrm{C}(15)-\mathrm{O}(1)$ & $-179.5(5)$ \\
\hline $\mathrm{C}(17)-\mathrm{C}(16)-\mathrm{C}(15)-\mathrm{O}(1)$ & $0.7(8)$ \\
\hline $\mathrm{C}(1)-\mathrm{O}(1)-\mathrm{C}(15)-\mathrm{C}(14)$ & $179.2(5)$ \\
\hline $\mathrm{C}(1)-\mathrm{O}(1)-\mathrm{C}(15)-\mathrm{C}(16)$ & $0.4(8)$ \\
\hline$C(3)-C(2)-C(1)-C(18)$ & $-0.3(9)$ \\
\hline $\mathrm{C}(3)-\mathrm{C}(2)-\mathrm{C}(1)-\mathrm{O}(1)$ & $178.6(5)$ \\
\hline $\mathrm{C}(17)-\mathrm{C}(18)-\mathrm{C}(1)-\mathrm{C}(2)$ & $-179.9(5)$ \\
\hline $\mathrm{C}(5)-\mathrm{C}(18)-\mathrm{C}(1)-\mathrm{C}(2)$ & $1.3(8)$ \\
\hline $\mathrm{C}(17)-\mathrm{C}(18)-\mathrm{C}(1)-\mathrm{O}(1)$ & $1.2(8)$ \\
\hline $\mathrm{C}(5)-\mathrm{C}(18)-\mathrm{C}(1)-\mathrm{O}(1)$ & $-177.6(5)$ \\
\hline $\mathrm{C}(15)-\mathrm{O}(1)-\mathrm{C}(1)-\mathrm{C}(2)$ & $179.7(5)$ \\
\hline $\mathrm{C}(15)-\mathrm{O}(1)-\mathrm{C}(1)-\mathrm{C}(18)$ & $-1.4(7)$ \\
\hline $\mathrm{C}(12)-\mathrm{C}(11)-\mathrm{C}(10)-\mathrm{C}(9)$ & $-178.9(6)$ \\
\hline $\mathrm{C}(16)-\mathrm{C}(11)-\mathrm{C}(10)-\mathrm{C}(9)$ & $-2.0(8)$ \\
\hline $\mathrm{C}(11)-\mathrm{C}(10)-\mathrm{C}(9)-\mathrm{C}(8)$ & $3.0(9)$ \\
\hline$C(7)-C(8)-C(9)-C(10)$ & $177.7(6)$ \\
\hline $\mathrm{C}(17)-\mathrm{C}(8)-\mathrm{C}(9)-\mathrm{C}(10)$ & $-3.1(9)$ \\
\hline $\mathrm{C}(16)-\mathrm{C}(11)-\mathrm{C}(12)-\mathrm{C}(13)$ & $1.5(10)$ \\
\hline $\mathrm{C}(10)-\mathrm{C}(11)-\mathrm{C}(12)-\mathrm{C}(13)$ & $178.4(6)$ \\
\hline$C(4)-C(5)-C(6)-C(7)$ & $-179.8(6)$ \\
\hline $\mathrm{C}(18)-\mathrm{C}(5)-\mathrm{C}(6)-\mathrm{C}(7)$ & $-1.7(8)$ \\
\hline $\mathrm{C}(16)-\mathrm{C}(15)-\mathrm{C}(14)-\mathrm{C}(13)$ & $-2.1(9)$ \\
\hline $\mathrm{O}(1)-\mathrm{C}(15)-\mathrm{C}(14)-\mathrm{C}(13)$ & $179.1(6)$ \\
\hline $\mathrm{C}(5)-\mathrm{C}(6)-\mathrm{C}(7)-\mathrm{C}(8)$ & $2.0(10)$ \\
\hline $\mathrm{C}(9)-\mathrm{C}(8)-\mathrm{C}(7)-\mathrm{C}(6)$ & $177.7(6)$ \\
\hline $\mathrm{C}(17)-\mathrm{C}(8)-\mathrm{C}(7)-\mathrm{C}(6)$ & $-1.5(9)$ \\
\hline $\mathrm{C}(18)-\mathrm{C}(5)-\mathrm{C}(4)-\mathrm{C}(3)$ & $1.7(9)$ \\
\hline$C(6)-C(5)-C(4)-C(3)$ & $179.8(6)$ \\
\hline $\mathrm{C}(1)-\mathrm{C}(2)-\mathrm{C}(3)-\mathrm{C}(4)$ & $0.0(9)$ \\
\hline $\mathrm{C}(5)-\mathrm{C}(4)-\mathrm{C}(3)-\mathrm{C}(2)$ & $-0.7(10)$ \\
\hline$C(11)-C(12)-C(13)-C(14)$ & $-2.0(11)$ \\
\hline$C(15)-C(14)-C(13)-C(12)$ & $2.2(10)$ \\
\hline $\mathrm{C}(29)-\mathrm{C}(34)-\mathrm{C}(35)-\mathrm{C}(26)$ & $1.7(8)$ \\
\hline $\mathrm{C}(33)-\mathrm{C}(34)-\mathrm{C}(35)-\mathrm{C}(26)$ & $179.0(6)$ \\
\hline
\end{tabular}




\begin{tabular}{|c|c|}
\hline$C(29)-C(34)-C(35)-C(36)$ & $-177.6(5)$ \\
\hline $\mathrm{C}(33)-\mathrm{C}(34)-\mathrm{C}(35)-\mathrm{C}(36)$ & $-0.2(7)$ \\
\hline $\mathrm{C}(19)-\mathrm{C}(36)-\mathrm{C}(35)-\mathrm{C}(26)$ & $179.5(6)$ \\
\hline$C(23)-C(36)-C(35)-C(26)$ & $1.1(7)$ \\
\hline $\mathrm{C}(19)-\mathrm{C}(36)-\mathrm{C}(35)-\mathrm{C}(34)$ & $-1.3(7)$ \\
\hline $\mathrm{C}(23)-\mathrm{C}(36)-\mathrm{C}(35)-\mathrm{C}(34)$ & $-179.7(5)$ \\
\hline $\mathrm{C}(19)-\mathrm{O}(2)-\mathrm{C}(33)-\mathrm{C}(32)$ & $178.5(6)$ \\
\hline $\mathrm{C}(19)-\mathrm{O}(2)-\mathrm{C}(33)-\mathrm{C}(34)$ & $-2.1(7)$ \\
\hline $\mathrm{C}(35)-\mathrm{C}(34)-\mathrm{C}(33)-\mathrm{C}(32)$ & $-178.7(5)$ \\
\hline $\mathrm{C}(29)-\mathrm{C}(34)-\mathrm{C}(33)-\mathrm{C}(32)$ & $-1.3(8)$ \\
\hline $\mathrm{C}(35)-\mathrm{C}(34)-\mathrm{C}(33)-\mathrm{O}(2)$ & $1.9(8)$ \\
\hline $\mathrm{C}(29)-\mathrm{C}(34)-\mathrm{C}(33)-\mathrm{O}(2)$ & $179.3(5)$ \\
\hline $\mathrm{C}(35)-\mathrm{C}(34)-\mathrm{C}(29)-\mathrm{C}(30)$ & $179.2(5)$ \\
\hline $\mathrm{C}(33)-\mathrm{C}(34)-\mathrm{C}(29)-\mathrm{C}(30)$ & $1.7(8)$ \\
\hline $\mathrm{C}(35)-\mathrm{C}(34)-\mathrm{C}(29)-\mathrm{C}(28)$ & $-2.6(8)$ \\
\hline $\mathrm{C}(33)-\mathrm{C}(34)-\mathrm{C}(29)-\mathrm{C}(28)$ & $-180.0(5)$ \\
\hline $\mathrm{C}(33)-\mathrm{O}(2)-\mathrm{C}(19)-\mathrm{C}(20)$ & $179.5(5)$ \\
\hline $\mathrm{C}(33)-\mathrm{O}(2)-\mathrm{C}(19)-\mathrm{C}(36)$ & $0.6(8)$ \\
\hline $\mathrm{C}(23)-\mathrm{C}(36)-\mathrm{C}(19)-\mathrm{O}(2)$ & $179.5(5)$ \\
\hline $\mathrm{C}(35)-\mathrm{C}(36)-\mathrm{C}(19)-\mathrm{O}(2)$ & $1.1(8)$ \\
\hline $\mathrm{C}(23)-\mathrm{C}(36)-\mathrm{C}(19)-\mathrm{C}(20)$ & $0.6(8)$ \\
\hline $\mathrm{C}(35)-\mathrm{C}(36)-\mathrm{C}(19)-\mathrm{C}(20)$ & $-177.7(5)$ \\
\hline$C(19)-C(36)-C(23)-C(24)$ & $179.2(6)$ \\
\hline $\mathrm{C}(35)-\mathrm{C}(36)-\mathrm{C}(23)-\mathrm{C}(24)$ & $-2.5(8)$ \\
\hline $\mathrm{C}(19)-\mathrm{C}(36)-\mathrm{C}(23)-\mathrm{C}(22)$ & $0.2(8)$ \\
\hline $\mathrm{C}(35)-\mathrm{C}(36)-\mathrm{C}(23)-\mathrm{C}(22)$ & $178.5(5)$ \\
\hline $\mathrm{C}(34)-\mathrm{C}(35)-\mathrm{C}(26)-\mathrm{C}(27)$ & $-1.1(8)$ \\
\hline$C(36)-C(35)-C(26)-C(27)$ & $178.2(5)$ \\
\hline $\mathrm{C}(34)-\mathrm{C}(35)-\mathrm{C}(26)-\mathrm{C}(25)$ & $-178.4(5)$ \\
\hline $\mathrm{C}(36)-\mathrm{C}(35)-\mathrm{C}(26)-\mathrm{C}(25)$ & $0.8(8)$ \\
\hline $\mathrm{O}(2)-\mathrm{C}(33)-\mathrm{C}(32)-\mathrm{C}(31)$ & $-178.6(6)$ \\
\hline $\mathrm{C}(34)-\mathrm{C}(33)-\mathrm{C}(32)-\mathrm{C}(31)$ & $2.0(9)$ \\
\hline $\mathrm{O}(2)-\mathrm{C}(19)-\mathrm{C}(20)-\mathrm{C}(21)$ & $-179.6(5)$ \\
\hline$C(36)-C(19)-C(20)-C(21)$ & $-0.7(8)$ \\
\hline $\mathrm{C}(24)-\mathrm{C}(23)-\mathrm{C}(22)-\mathrm{C}(21)$ & $-179.9(6)$ \\
\hline $\mathrm{C}(36)-\mathrm{C}(23)-\mathrm{C}(22)-\mathrm{C}(21)$ & $-1.0(9)$ \\
\hline $\mathrm{C}(30)-\mathrm{C}(29)-\mathrm{C}(28)-\mathrm{C}(27)$ & $-178.7(6)$ \\
\hline $\mathrm{C}(34)-\mathrm{C}(29)-\mathrm{C}(28)-\mathrm{C}(27)$ & $3.1(8)$ \\
\hline $\mathrm{C}(29)-\mathrm{C}(28)-\mathrm{C}(27)-\mathrm{C}(26)$ & $-2.6(8)$ \\
\hline $\mathrm{C}(35)-\mathrm{C}(26)-\mathrm{C}(27)-\mathrm{C}(28)$ & $1.5(8)$ \\
\hline $\mathrm{C}(25)-\mathrm{C}(26)-\mathrm{C}(27)-\mathrm{C}(28)$ & $178.8(6)$ \\
\hline $\mathrm{C}(34)-\mathrm{C}(29)-\mathrm{C}(30)-\mathrm{C}(31)$ & $-2.9(10)$ \\
\hline $\mathrm{C}(28)-\mathrm{C}(29)-\mathrm{C}(30)-\mathrm{C}(31)$ & $178.9(6)$ \\
\hline$C(35)-C(26)-C(25)-C(24)$ & $-1.3(9)$ \\
\hline$C(27)-C(26)-C(25)-C(24)$ & $-178.6(5)$ \\
\hline $\mathrm{C}(29)-\mathrm{C}(30)-\mathrm{C}(31)-\mathrm{C}(32)$ & $3.6(10)$ \\
\hline $\mathrm{C}(33)-\mathrm{C}(32)-\mathrm{C}(31)-\mathrm{C}(30)$ & $-3.2(10)$ \\
\hline $\mathrm{C}(36)-\mathrm{C}(23)-\mathrm{C}(24)-\mathrm{C}(25)$ & $2.0(9)$ \\
\hline $\mathrm{C}(22)-\mathrm{C}(23)-\mathrm{C}(24)-\mathrm{C}(25)$ & $-179.1(6)$ \\
\hline$C(26)-C(25)-C(24)-C(23)$ & $-0.2(9)$ \\
\hline$C(23)-C(22)-C(21)-C(20)$ & $1.0(10)$ \\
\hline $\mathrm{C}(19)-\mathrm{C}(20)-\mathrm{C}(21)-\mathrm{C}(22)$ & $-0.2(9)$ \\
\hline
\end{tabular}


X-Ray data for 9,10-bis[bis(trimethylsilylethynyl)methylene]-9,10-dihydroanthracene (17):
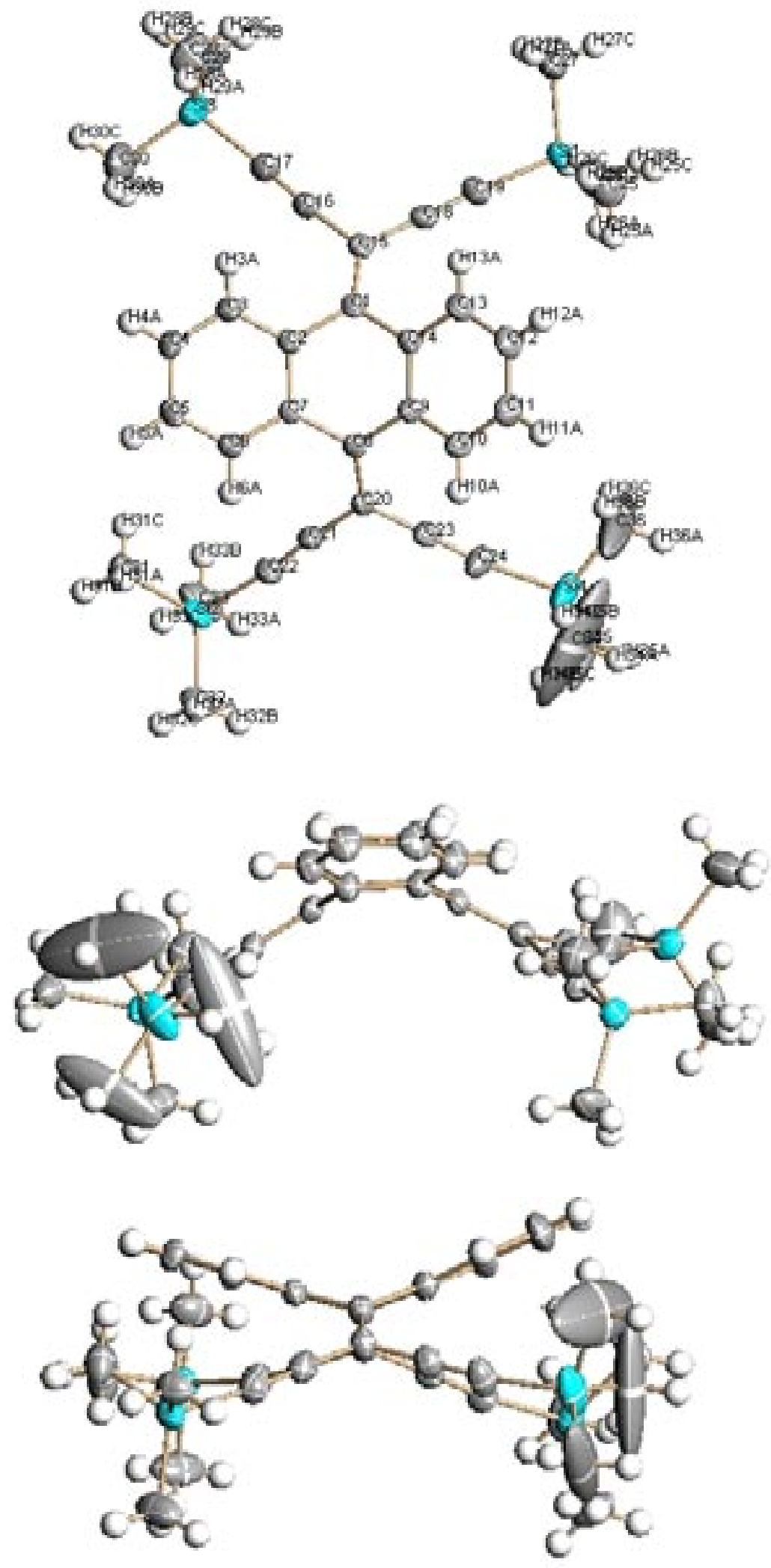

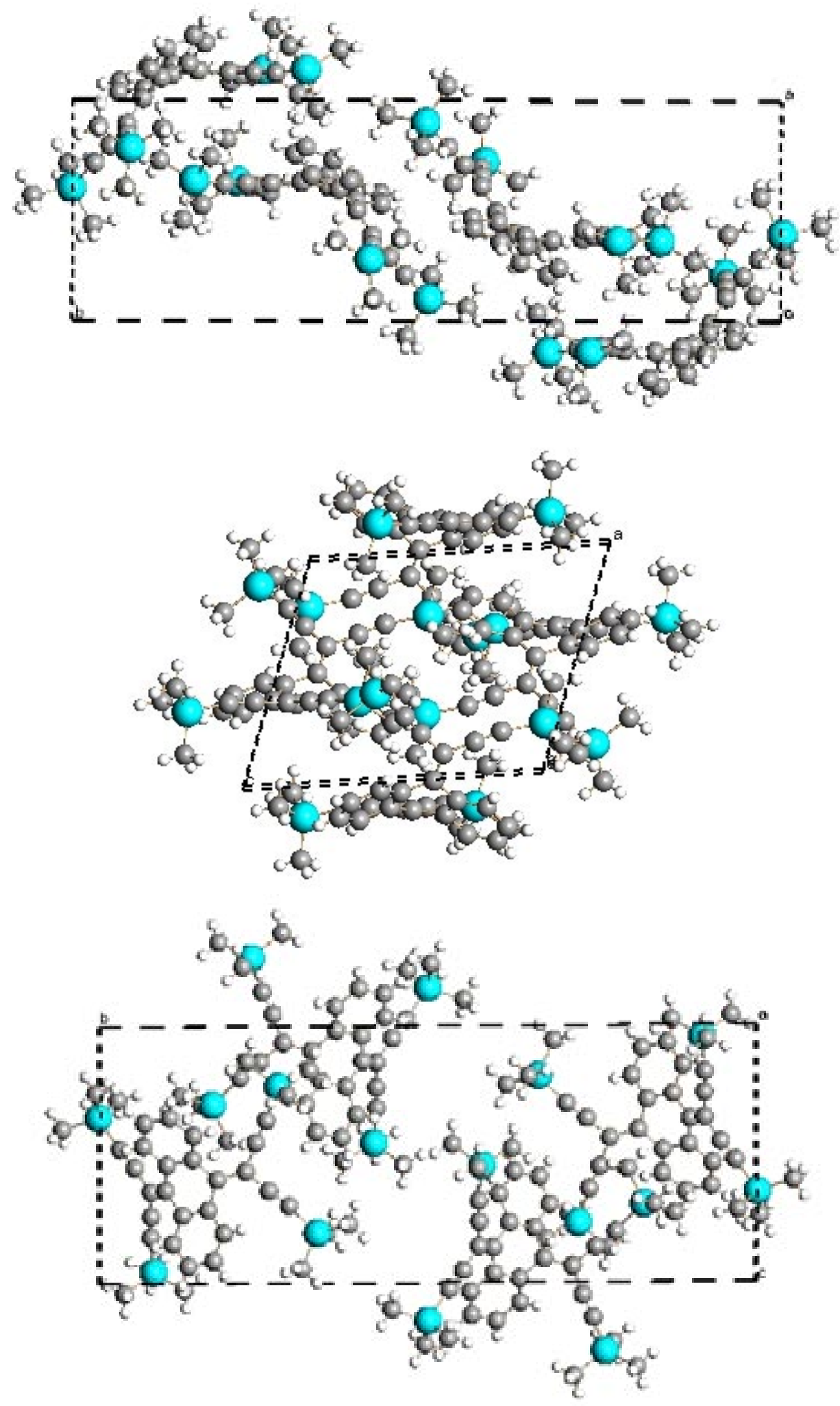

page S29 
Table 1. Crystal data and structure refinement for 9,10-bis[bis(trimethylsilylethynyl)methylene]-9-10dihydroanthracene (17).

Identification code $\quad$ pmd02t

Empirical formula

C36 H44 Si4

Formula weight

Temperature

Wavelength

Crystal system

Space group

Unit cell dimensions

589.07

193(2) K

$0.71073 \AA$

Monoclinic

$\mathrm{P} 2(1) / \mathrm{n}$

$\mathrm{a}=10.0038(6) \AA$

$\alpha=90^{\circ}$.

$\mathrm{b}=30.3064(19) \AA$

$\beta=109.6960(10)^{\circ}$.

$\mathrm{c}=12.6144(8) \AA$ $\gamma=90^{\circ}$.

Volume

3600.7(4) $\AA^{3}$

Z

Density (calculated)

4

Absorption coefficient

$1.087 \mathrm{Mg} / \mathrm{m}^{3}$

$\mathrm{F}(000)$

$0.187 \mathrm{~mm}^{-1}$

Crystal size

Theta range for data collection

1264

Index ranges

Reflections collected

Independent reflections

Completeness to theta $=28.36^{\circ}$

$0.65 \times 0.30 \times 0.05 \mathrm{~mm}^{3}$

1.34 to $28.36^{\circ}$.

$-13<=\mathrm{h}<=11,-40<=\mathrm{k}<=37,-16<=1<=16$

26801

$8987[\mathrm{R}(\mathrm{int})=0.0762]$

Absorption correction

$99.7 \%$

Refinement method

Data / restraints / parameters

None

Goodness-of-fit on $\mathrm{F}^{2}$

Full-matrix least-squares on $\mathrm{F}^{2}$

8987 / 0 / 361

Final R indices [I $>2$ sigma(I)]

1.026

$\mathrm{R}$ indices (all data)

$\mathrm{R} 1=0.0618, \mathrm{wR} 2=0.1690$

$\mathrm{R} 1=0.0934, \mathrm{wR} 2=0.1855$

Largest diff. peak and hole

0.746 and -0.679 e. $\AA^{-3}$

Table 2. Atomic coordinates $\left(\times 10^{4}\right)$ and equivalent isotropic displacement parameters $\left(\AA^{2} \times 10^{3}\right)$ for 9,10-bis[bis(trimethylsilylethynyl)methylene]-9-10-dihydroanthracene (17). U(eq) is defined as one third of the trace of the orthogonalized $\mathrm{U}^{\mathrm{ij}}$ tensor.

\begin{tabular}{|c|c|c|c|c|}
\hline & $\mathrm{x}$ & $\mathrm{y}$ & $\mathrm{z}$ & $\mathrm{U}(\mathrm{eq})$ \\
\hline$\overline{C(24)}$ & $6944(3)$ & $4230(1)$ & $6816(2)$ & $46(1)$ \\
\hline$C(26)$ & $5407(3)$ & $1559(1)$ & $6958(3)$ & $63(1)$ \\
\hline $\operatorname{Si}(1)$ & $3623(1)$ & 1711(1) & $6947(1)$ & $35(1)$ \\
\hline $\operatorname{Si}(2)$ & $8947(1)$ & $5017(1)$ & $11473(1)$ & $39(1)$ \\
\hline $\operatorname{Si}(3)$ & 3593(1) & $2305(1)$ & $12665(1)$ & $44(1)$ \\
\hline $\mathrm{Si}(4)$ & $7218(1)$ & $4188(1)$ & $5447(1)$ & $67(1)$ \\
\hline $\mathrm{C}(9)$ & $3987(2)$ & $3757(1)$ & $7653(2)$ & $27(1)$ \\
\hline $\mathrm{C}(2)$ & $3838(2)$ & $3604(1)$ & $9840(2)$ & $26(1)$ \\
\hline $\mathrm{C}(3)$ & $3366(3)$ & 3593(1) & $10758(2)$ & $31(1)$ \\
\hline$C(7)$ & $4486(2)$ & 3992(1) & $9624(2)$ & $25(1)$ \\
\hline $\mathrm{C}(1)$ & $3651(2)$ & $3229(1)$ & $9048(2)$ & $26(1)$ \\
\hline $\mathrm{C}(8)$ & 4927(2) & $4010(1)$ & $8622(2)$ & $26(1)$ \\
\hline $\mathrm{C}(19)$ & $3767(3)$ & $2137(1)$ & $8015(2)$ & $37(1)$ \\
\hline$C(15)$ & $3737(2)$ & 2797(1) & $9365(2)$ & $29(1)$ \\
\hline $\mathrm{C}(20)$ & $6103(3)$ & $4230(1)$ & $8583(2)$ & $30(1)$ \\
\hline
\end{tabular}




\begin{tabular}{|c|c|c|c|c|}
\hline$C(5)$ & $4160(3)$ & $4336(1)$ & $11245(2)$ & $34(1)$ \\
\hline $\mathrm{C}(14)$ & $3368(2)$ & $3368(1)$ & $7867(2)$ & $27(1)$ \\
\hline$C(18)$ & $3738(3)$ & $2443(1)$ & $8610(2)$ & $32(1)$ \\
\hline$C(6)$ & $4630(2)$ & $4353(1)$ & $10343(2)$ & $30(1)$ \\
\hline $\mathrm{C}(21)$ & $7055(3)$ & $4471(1)$ & $9521(2)$ & $30(1)$ \\
\hline $\mathrm{C}(13)$ & $2424(3)$ & $3138(1)$ & $6962(2)$ & $34(1)$ \\
\hline $\mathrm{C}(4)$ & $3526(3)$ & $3954(1)$ & $11460(2)$ & $34(1)$ \\
\hline $\mathrm{C}(23)$ & $6550(3)$ & $4225(1)$ & $7617(2)$ & $35(1)$ \\
\hline$C(22)$ & $7855(3)$ & 4691(1) & $10246(2)$ & $36(1)$ \\
\hline$C(16)$ & $3851(3)$ & $2642(1)$ & $10479(2)$ & $34(1)$ \\
\hline $\mathrm{C}(17)$ & $3860(3)$ & 2483(1) & $11347(2)$ & $40(1)$ \\
\hline$C(10)$ & $3616(3)$ & 3911(1) & $6550(2)$ & $37(1)$ \\
\hline$C(12)$ & 2094(3) & $3292(1)$ & $5879(2)$ & $42(1)$ \\
\hline $\mathrm{C}(27)$ & $2713(4)$ & $1231(1)$ & $7289(3)$ & $57(1)$ \\
\hline $\mathrm{C}(11)$ & $2682(3)$ & $3676(1)$ & $5671(2)$ & $44(1)$ \\
\hline$C(25)$ & $2561(4)$ & $1945(1)$ & $5568(2)$ & $59(1)$ \\
\hline $\mathrm{C}(28)$ & $1697(3)$ & $2172(1)$ & $12324(3)$ & $65(1)$ \\
\hline C(29) & $4686(4)$ & $1821(1)$ & $13284(3)$ & $79(1)$ \\
\hline$C(30)$ & $4132(5)$ & $2779(1)$ & $13631(3)$ & $92(1)$ \\
\hline $\mathrm{C}(32)$ & $9327(3)$ & $5567(1)$ & $11026(2)$ & $47(1)$ \\
\hline $\mathrm{C}(31)$ & $7857(4)$ & $5061(1)$ & $12407(2)$ & $56(1)$ \\
\hline C(33) & $10630(4)$ & $4715(1)$ & $12161(3)$ & $67(1)$ \\
\hline$C(35)$ & $8976(6)$ & $4249(3)$ & $5535(4)$ & $235(5)$ \\
\hline$C(36)$ & $6342(13)$ & $3727(4)$ & $4764(6)$ & $389(11)$ \\
\hline$C(34)$ & $6250(12)$ & $4633(4)$ & $4612(6)$ & $382(11)$ \\
\hline
\end{tabular}

Table 3. Bond lengths $[\AA]$ and angles $\left[^{\circ}\right]$ for 9,10-bis[bis(trimethylsilylethynyl)methylene]-9-10dihydroanthracene (17).

\begin{tabular}{llll}
\hline $\mathrm{C}(24)-\mathrm{C}(23)$ & $1.203(4)$ & $\mathrm{C}(2)-\mathrm{C}(7)$ & $1.413(3)$ \\
$\mathrm{C}(24)-\mathrm{Si}(4)$ & $1.842(3)$ & $\mathrm{C}(2)-\mathrm{C}(1)$ & $1.482(3)$ \\
$\mathrm{C}(26)-\mathrm{Si}(1)$ & $1.839(3)$ & $\mathrm{C}(3)-\mathrm{C}(4)$ & $1.383(3)$ \\
$\mathrm{Si}(1)-\mathrm{C}(19)$ & $1.838(3)$ & $\mathrm{C}(7)-\mathrm{C}(6)$ & $1.398(3)$ \\
$\mathrm{Si}(1)-\mathrm{C}(27)$ & $1.842(3)$ & $\mathrm{C}(7)-\mathrm{C}(8)$ & $1.474(3)$ \\
$\mathrm{Si}(1)-\mathrm{C}(25)$ & $1.848(3)$ & $\mathrm{C}(1)-\mathrm{C}(15)$ & $1.364(3)$ \\
$\mathrm{Si}(2)-\mathrm{C}(32)$ & $1.840(3)$ & $\mathrm{C}(1)-\mathrm{C}(14)$ & $1.367(3)$ \\
$\mathrm{Si}(2)-\mathrm{C}(22)$ & $1.852(2)$ & $\mathrm{C}(8)-\mathrm{C}(20)$ & $1.199(3)$ \\
$\mathrm{Si}(2)-\mathrm{C}(33)$ & $1.856(3)$ & $\mathrm{C}(19)-\mathrm{C}(18)$ & $1.435(3)$ \\
$\mathrm{Si}(2)-\mathrm{C}(31)$ & $1.860(3)$ & $\mathrm{C}(15)-\mathrm{C}(18)$ & $1.449(3)$ \\
$\mathrm{Si}(3)-\mathrm{C}(29)$ & $1.838(3)$ & $\mathrm{C}(15)-\mathrm{C}(16)$ & $1.431(3)$ \\
$\mathrm{Si}(3)-\mathrm{C}(30)$ & $1.843(4)$ & $\mathrm{C}(20)-\mathrm{C}(23)$ & $1.443(3)$ \\
$\mathrm{Si}(3)-\mathrm{C}(28)$ & $1.843(3)$ & $\mathrm{C}(20)-\mathrm{C}(21)$ & $1.371(3)$ \\
$\mathrm{Si}(3)-\mathrm{C}(17)$ & $1.850(3)$ & $\mathrm{C}(5)-\mathrm{C}(6)$ & $1.398(3)$ \\
$\mathrm{Si}(4)-\mathrm{C}(36)$ & $1.718(6)$ & $\mathrm{C}(5)-\mathrm{C}(4)$ & $1.194(3)$ \\
$\mathrm{Si}(4)-\mathrm{C}(35)$ & $1.735(6)$ & $\mathrm{C}(21)-\mathrm{C}(22)$ & $1.375(3)$ \\
$\mathrm{Si}(4)-\mathrm{C}(34)$ & $1.783(8)$ & $\mathrm{C}(13)-\mathrm{C}(12)$ & $1.195(3)$ \\
$\mathrm{C}(9)-\mathrm{C}(10)$ & $1.394(3)$ & $\mathrm{C}(16)-\mathrm{C}(17)$ & $1.383(3)$ \\
$\mathrm{C}(9)-\mathrm{C}(14)$ & $1.399(3)$ & $\mathrm{C}(10)-\mathrm{C}(11)$ & $1.368(4)$ \\
$\mathrm{C}(9)-\mathrm{C}(8)$ & $1.480(3)$ & $\mathrm{C}(12)-\mathrm{C}(11)$ & \\
$\mathrm{C}(2)-\mathrm{C}(3)$ & $1.390(3)$ & &
\end{tabular}




\begin{tabular}{|c|c|c|c|}
\hline$C(23)-C(24)-S i(4)$ & $169.0(2)$ & $C(6)-C(7)-C(2)$ & $118.7(2)$ \\
\hline $\mathrm{C}(19)-\mathrm{Si}(1)-\mathrm{C}(26)$ & $109.55(14)$ & $C(6)-C(7)-C(8)$ & $122.2(2)$ \\
\hline $\mathrm{C}(19)-\mathrm{Si}(1)-\mathrm{C}(27)$ & $107.81(13)$ & $\mathrm{C}(2)-\mathrm{C}(7)-\mathrm{C}(8)$ & $119.05(19)$ \\
\hline $\mathrm{C}(26)-\mathrm{Si}(1)-\mathrm{C}(27)$ & $110.86(16)$ & $\mathrm{C}(15)-\mathrm{C}(1)-\mathrm{C}(14)$ & $122.7(2)$ \\
\hline $\mathrm{C}(19)-\mathrm{Si}(1)-\mathrm{C}(25)$ & $107.37(13)$ & $\mathrm{C}(15)-\mathrm{C}(1)-\mathrm{C}(2)$ & $123.8(2)$ \\
\hline $\mathrm{C}(26)-\mathrm{Si}(1)-\mathrm{C}(25)$ & $110.59(17)$ & $\mathrm{C}(14)-\mathrm{C}(1)-\mathrm{C}(2)$ & $113.55(18)$ \\
\hline $\mathrm{C}(27)-\mathrm{Si}(1)-\mathrm{C}(25)$ & $110.55(15)$ & $C(20)-C(8)-C(7)$ & $124.0(2)$ \\
\hline $\mathrm{C}(32)-\mathrm{Si}(2)-\mathrm{C}(22)$ & $110.66(12)$ & $\mathrm{C}(20)-\mathrm{C}(8)-\mathrm{C}(9)$ & $122.5(2)$ \\
\hline $\mathrm{C}(32)-\mathrm{Si}(2)-\mathrm{C}(33)$ & $109.99(15)$ & $\mathrm{C}(7)-\mathrm{C}(8)-\mathrm{C}(9)$ & $113.5(2)$ \\
\hline $\mathrm{C}(22)-\mathrm{Si}(2)-\mathrm{C}(33)$ & $108.26(13)$ & $\mathrm{C}(18)-\mathrm{C}(19)-\mathrm{Si}(1)$ & $172.0(2)$ \\
\hline $\mathrm{C}(32)-\mathrm{Si}(2)-\mathrm{C}(31)$ & $110.84(14)$ & $C(1)-C(15)-C(18)$ & $122.5(2)$ \\
\hline$C(22)-\operatorname{Si}(2)-C(31)$ & $105.13(13)$ & $\mathrm{C}(1)-\mathrm{C}(15)-\mathrm{C}(16)$ & $125.0(2)$ \\
\hline $\mathrm{C}(33)-\mathrm{Si}(2)-\mathrm{C}(31)$ & $111.85(17)$ & $\mathrm{C}(18)-\mathrm{C}(15)-\mathrm{C}(16)$ & $112.6(2)$ \\
\hline $\mathrm{C}(29)-\mathrm{Si}(3)-\mathrm{C}(30)$ & $110.0(2)$ & $\mathrm{C}(8)-\mathrm{C}(20)-\mathrm{C}(23)$ & $123.0(2)$ \\
\hline $\mathrm{C}(29)-\mathrm{Si}(3)-\mathrm{C}(28)$ & $109.99(17)$ & $\mathrm{C}(8)-\mathrm{C}(20)-\mathrm{C}(21)$ & $123.8(2)$ \\
\hline $\mathrm{C}(30)-\mathrm{Si}(3)-\mathrm{C}(28)$ & $112.0(2)$ & $\mathrm{C}(23)-\mathrm{C}(20)-\mathrm{C}(21)$ & $113.2(2)$ \\
\hline $\mathrm{C}(29)-\mathrm{Si}(3)-\mathrm{C}(17)$ & $111.62(15)$ & $C(6)-C(5)-C(4)$ & $120.0(2)$ \\
\hline $\mathrm{C}(30)-\mathrm{Si}(3)-\mathrm{C}(17)$ & $105.57(15)$ & $\mathrm{C}(13)-\mathrm{C}(14)-\mathrm{C}(9)$ & $118.9(2)$ \\
\hline $\mathrm{C}(28)-\mathrm{Si}(3)-\mathrm{C}(17)$ & $107.62(14)$ & $\mathrm{C}(13)-\mathrm{C}(14)-\mathrm{C}(1)$ & $121.9(2)$ \\
\hline $\mathrm{C}(36)-\mathrm{Si}(4)-\mathrm{C}(35)$ & $117.0(5)$ & $\mathrm{C}(9)-\mathrm{C}(14)-\mathrm{C}(1)$ & $119.08(18)$ \\
\hline $\mathrm{C}(36)-\mathrm{Si}(4)-\mathrm{C}(34)$ & $103.6(6)$ & $\mathrm{C}(19)-\mathrm{C}(18)-\mathrm{C}(15)$ & $177.4(3)$ \\
\hline $\mathrm{C}(35)-\mathrm{Si}(4)-\mathrm{C}(34)$ & $107.1(5)$ & $\mathrm{C}(5)-\mathrm{C}(6)-\mathrm{C}(7)$ & $121.3(2)$ \\
\hline $\mathrm{C}(36)-\mathrm{Si}(4)-\mathrm{C}(24)$ & $108.4(2)$ & $\mathrm{C}(22)-\mathrm{C}(21)-\mathrm{C}(20)$ & $175.6(3)$ \\
\hline $\mathrm{C}(35)-\mathrm{Si}(4)-\mathrm{C}(24)$ & $113.62(19)$ & $\mathrm{C}(12)-\mathrm{C}(13)-\mathrm{C}(14)$ & $120.8(2)$ \\
\hline $\mathrm{C}(34)-\mathrm{Si}(4)-\mathrm{C}(24)$ & $106.2(3)$ & $C(3)-C(4)-C(5)$ & $119.7(2)$ \\
\hline $\mathrm{C}(10)-\mathrm{C}(9)-\mathrm{C}(14)$ & $119.3(2)$ & $\mathrm{C}(24)-\mathrm{C}(23)-\mathrm{C}(20)$ & $178.3(3)$ \\
\hline $\mathrm{C}(10)-\mathrm{C}(9)-\mathrm{C}(8)$ & $122.1(2)$ & $\mathrm{C}(21)-\mathrm{C}(22)-\mathrm{Si}(2)$ & $172.9(2)$ \\
\hline $\mathrm{C}(14)-\mathrm{C}(9)-\mathrm{C}(8)$ & $118.44(19)$ & $\mathrm{C}(17)-\mathrm{C}(16)-\mathrm{C}(15)$ & $173.8(3)$ \\
\hline$C(3)-C(2)-C(7)$ & 119.1(2) & $\mathrm{C}(16)-\mathrm{C}(17)-\mathrm{Si}(3)$ & 169.4(3) \\
\hline $\mathrm{C}(3)-\mathrm{C}(2)-\mathrm{C}(1)$ & $122.9(2)$ & $\mathrm{C}(11)-\mathrm{C}(10)-\mathrm{C}(9)$ & $120.5(2)$ \\
\hline $\mathrm{C}(7)-\mathrm{C}(2)-\mathrm{C}(1)$ & $118.0(2)$ & $\mathrm{C}(11)-\mathrm{C}(12)-\mathrm{C}(13)$ & $120.3(2)$ \\
\hline $\mathrm{C}(4)-\mathrm{C}(3)-\mathrm{C}(2)$ & $121.1(2)$ & $\mathrm{C}(12)-\mathrm{C}(11)-\mathrm{C}(10)$ & $120.2(2)$ \\
\hline
\end{tabular}

Table 4. Anisotropic displacement parameters $\left(\AA^{2} \times 10^{3}\right)$ for 9,10-bis[bis(trimethylsilylethynyl)methylene]-9-10dihydroanthracene (17). The anisotropic displacement factor exponent takes the form: $-2 \pi^{2}\left[h^{2} a^{* 2} U^{11}+\ldots+2 h\right.$ $\mathrm{ka} \mathrm{a}^{*} \mathrm{U}^{12}$ ]

\begin{tabular}{lcccccc}
\hline & $\mathrm{U}^{11}$ & $\mathrm{U}^{22}$ & $\mathrm{U}^{33}$ & $\mathrm{U}^{23}$ & $\mathrm{U}^{13}$ & $\mathrm{U}^{12}$ \\
\hline $\mathrm{C}(24)$ & $44(2)$ & $65(2)$ & $32(1)$ & $-2(1)$ & $14(1)$ & $-16(1)$ \\
$\mathrm{C}(26)$ & $50(2)$ & $53(2)$ & $93(3)$ & $-18(2)$ & $31(2)$ & $4(2)$ \\
$\mathrm{Si}(1)$ & $39(1)$ & $30(1)$ & $37(1)$ & $-8(1)$ & $14(1)$ & $-3(1)$ \\
$\mathrm{Si}(2)$ & $41(1)$ & $31(1)$ & $38(1)$ & $-9(1)$ & $3(1)$ & $-3(1)$ \\
$\mathrm{Si}(3)$ & $50(1)$ & $46(1)$ & $39(1)$ & $12(1)$ & $19(1)$ & $7(1)$ \\
$\mathrm{Si}(4)$ & $61(1)$ & $113(1)$ & $32(1)$ & $-6(1)$ & $22(1)$ & $-29(1)$ \\
$\mathrm{C}(9)$ & $28(1)$ & $27(1)$ & $25(1)$ & $-2(1)$ & $8(1)$ & $-2(1)$ \\
$\mathrm{C}(2)$ & $27(1)$ & $24(1)$ & $26(1)$ & $-1(1)$ & $7(1)$ & $1(1)$ \\
$\mathrm{C}(3)$ & $35(1)$ & $27(1)$ & $33(1)$ & $-1(1)$ & $15(1)$ & $-2(1)$ \\
$\mathrm{C}(7)$ & $26(1)$ & $24(1)$ & $23(1)$ & $0(1)$ & $7(1)$ & $0(1)$ \\
$\mathrm{C}(1)$ & $24(1)$ & $27(1)$ & $28(1)$ & $-4(1)$ & $9(1)$ & $-4(1)$ \\
$\mathrm{C}(8)$ & $31(1)$ & $21(1)$ & $25(1)$ & $0(1)$ & $9(1)$ & $0(1)$ \\
$\mathrm{C}(19)$ & $41(2)$ & $28(1)$ & $41(1)$ & $-3(1)$ & $12(1)$ & $-1(1)$ \\
$\mathrm{C}(15)$ & $29(1)$ & $26(1)$ & $30(1)$ & $-3(1)$ & $9(1)$ & $-3(1)$ \\
$\mathrm{C}(20)$ & $37(1)$ & $26(1)$ & $26(1)$ & $0(1)$ & $10(1)$ & $-3(1)$ \\
& & & & & &
\end{tabular}




\begin{tabular}{lcccccc}
$\mathrm{C}(5)$ & $39(1)$ & $29(1)$ & $34(1)$ & $-8(1)$ & $14(1)$ & $1(1)$ \\
$\mathrm{C}(14)$ & $28(1)$ & $27(1)$ & $27(1)$ & $-4(1)$ & $9(1)$ & $-1(1)$ \\
$\mathrm{C}(18)$ & $34(1)$ & $27(1)$ & $34(1)$ & $1(1)$ & $10(1)$ & $-4(1)$ \\
$\mathrm{C}(6)$ & $31(1)$ & $24(1)$ & $33(1)$ & $-2(1)$ & $10(1)$ & $-2(1)$ \\
$\mathrm{C}(21)$ & $33(1)$ & $28(1)$ & $31(1)$ & $0(1)$ & $14(1)$ & $-4(1)$ \\
$\mathrm{C}(13)$ & $32(1)$ & $33(1)$ & $34(1)$ & $-5(1)$ & $8(1)$ & $-5(1)$ \\
$\mathrm{C}(4)$ & $39(1)$ & $37(1)$ & $31(1)$ & $-2(1)$ & $18(1)$ & $1(1)$ \\
$\mathrm{C}(23)$ & $36(1)$ & $35(1)$ & $32(1)$ & $-2(1)$ & $9(1)$ & $-10(1)$ \\
$\mathrm{C}(22)$ & $36(1)$ & $35(1)$ & $37(1)$ & $-4(1)$ & $12(1)$ & $-5(1)$ \\
$\mathrm{C}(16)$ & $42(1)$ & $25(1)$ & $37(1)$ & $-2(1)$ & $15(1)$ & $-1(1)$ \\
$\mathrm{C}(17)$ & $50(2)$ & $31(1)$ & $41(1)$ & $4(1)$ & $18(1)$ & $2(1)$ \\
$\mathrm{C}(10)$ & $40(1)$ & $39(1)$ & $29(1)$ & $3(1)$ & $8(1)$ & $-6(1)$ \\
$\mathrm{C}(12)$ & $41(2)$ & $47(2)$ & $31(1)$ & $-9(1)$ & $3(1)$ & $-8(1)$ \\
$\mathrm{C}(27)$ & $83(2)$ & $44(2)$ & $52(2)$ & $-14(1)$ & $32(2)$ & $-22(2)$ \\
$\mathrm{C}(11)$ & $46(2)$ & $54(2)$ & $25(1)$ & $1(1)$ & $4(1)$ & $-4(1)$ \\
$\mathrm{C}(25)$ & $66(2)$ & $64(2)$ & $43(2)$ & $0(1)$ & $12(2)$ & $-2(2)$ \\
$\mathrm{C}(28)$ & $49(2)$ & $86(3)$ & $63(2)$ & $18(2)$ & $22(2)$ & $8(2)$ \\
$\mathrm{C}(29)$ & $67(2)$ & $92(3)$ & $89(3)$ & $59(2)$ & $40(2)$ & $33(2)$ \\
$\mathrm{C}(30)$ & $139(4)$ & $91(3)$ & $52(2)$ & $-17(2)$ & $39(2)$ & $-29(3)$ \\
$\mathrm{C}(32)$ & $39(2)$ & $39(1)$ & $56(2)$ & $-5(1)$ & $7(1)$ & $-6(1)$ \\
$\mathrm{C}(31)$ & $79(2)$ & $47(2)$ & $41(2)$ & $-7(1)$ & $21(2)$ & $0(2)$ \\
$\mathrm{C}(33)$ & $55(2)$ & $43(2)$ & $80(2)$ & $-10(2)$ & $-8(2)$ & $4(2)$ \\
$\mathrm{C}(35)$ & $89(4)$ & $564(16)$ & $69(3)$ & $-99(6)$ & $49(3)$ & $-59(7)$ \\
$\mathrm{C}(36)$ & $560(20)$ & $547(18)$ & $168(7)$ & $-252(10)$ & $264(11)$ & $-472(17)$ \\
$\mathrm{C}(34)$ & $420(17)$ & $650(20)$ & $156(7)$ & $239(12)$ & $204(10)$ & $358(18)$ \\
& & & & & & \\
\hline
\end{tabular}

Table 5. Hydrogen coordinates ( $\left.\times 10^{4}\right)$ and isotropic displacement parameters $\left(\AA^{2} \times 10^{3}\right)$ for 9,10-bis[bis(trimethylsilylethynyl)methylene]-9-10-dihydroanthracene (17).

\begin{tabular}{|c|c|c|c|c|}
\hline & $\mathrm{x}$ & $\mathrm{y}$ & $\mathrm{z}$ & $\mathrm{U}(\mathrm{eq})$ \\
\hline $\mathrm{H}(26 \mathrm{~A})$ & 5864 & 1813 & 6782 & 95 \\
\hline $\mathrm{H}(26 \mathrm{~B})$ & 5332 & 1333 & 6407 & 95 \\
\hline $\mathrm{H}(26 \mathrm{C})$ & 5958 & 1450 & 7690 & 95 \\
\hline $\mathrm{H}(3 \mathrm{~A})$ & 2936 & 3339 & 10901 & 37 \\
\hline $\mathrm{H}(5 \mathrm{~A})$ & 4265 & 4580 & 11714 & 40 \\
\hline $\mathrm{H}(6 \mathrm{~A})$ & 5053 & 4610 & 10207 & 35 \\
\hline $\mathrm{H}(13 \mathrm{~A})$ & 2014 & 2877 & 7094 & 41 \\
\hline $\mathrm{H}(4 \mathrm{~A})$ & 3211 & 3941 & 12074 & 41 \\
\hline $\mathrm{H}(10 \mathrm{~A})$ & 4000 & 4174 & 6405 & 44 \\
\hline $\mathrm{H}(12 \mathrm{~A})$ & 1467 & 3135 & 5285 & 51 \\
\hline $\mathrm{H}(27 \mathrm{~A})$ & 1786 & 1317 & 7280 & 86 \\
\hline $\mathrm{H}(27 \mathrm{~B})$ & 3254 & 1123 & 8024 & 86 \\
\hline $\mathrm{H}(27 \mathrm{C})$ & 2624 & 1003 & 6742 & 86 \\
\hline $\mathrm{H}(11 \mathrm{~A})$ & 2453 & 3779 & 4937 & 52 \\
\hline $\mathrm{H}(25 \mathrm{~A})$ & 3049 & 2194 & 5403 & 89 \\
\hline $\mathrm{H}(25 \mathrm{~B})$ & 1652 & 2036 & 5589 & 89 \\
\hline $\mathrm{H}(25 \mathrm{C})$ & 2431 & 1725 & 4993 & 89 \\
\hline $\mathrm{H}(28 \mathrm{~A})$ & 1134 & 2426 & 12005 & 98 \\
\hline $\mathrm{H}(28 \mathrm{~B})$ & 1522 & 2085 & 12998 & 98 \\
\hline $\mathrm{H}(28 \mathrm{C})$ & 1447 & 1933 & 11791 & 98 \\
\hline $\mathrm{H}(29 \mathrm{~A})$ & 5671 & 1893 & 13455 & 119 \\
\hline
\end{tabular}




\begin{tabular}{|c|c|c|c|c|}
\hline $\mathrm{H}(29 \mathrm{~B})$ & 4438 & 1581 & 12758 & 119 \\
\hline $\mathrm{H}(29 \mathrm{C})$ & 4519 & 1737 & 13962 & 119 \\
\hline $\mathrm{H}(30 \mathrm{~A})$ & 3557 & 3030 & 13302 & 138 \\
\hline $\mathrm{H}(30 \mathrm{~B})$ & 5112 & 2847 & 13761 & 138 \\
\hline $\mathrm{H}(30 \mathrm{C})$ & 4007 & 2708 & 14333 & 138 \\
\hline $\mathrm{H}(32 \mathrm{~A})$ & 8452 & 5722 & 10673 & 70 \\
\hline $\mathrm{H}(32 \mathrm{~B})$ & 9827 & 5536 & 10500 & 70 \\
\hline $\mathrm{H}(32 \mathrm{C})$ & 9902 & 5730 & 11671 & 70 \\
\hline $\mathrm{H}(31 \mathrm{~A})$ & 7000 & 5220 & 12025 & 84 \\
\hline $\mathrm{H}(31 \mathrm{~B})$ & 8385 & 5215 & 13083 & 84 \\
\hline $\mathrm{H}(31 \mathrm{C})$ & 7625 & 4771 & 12595 & 84 \\
\hline$H(33 A)$ & 11162 & 4698 & 11655 & 101 \\
\hline $\mathrm{H}(33 \mathrm{~B})$ & 10421 & 4422 & 12350 & 101 \\
\hline $\mathrm{H}(33 \mathrm{C})$ & 11178 & 4867 & 12834 & 101 \\
\hline $\mathrm{H}(35 \mathrm{~A})$ & 9046 & 4225 & 4796 & 353 \\
\hline $\mathrm{H}(35 \mathrm{~B})$ & 9541 & 4023 & 6011 & 353 \\
\hline $\mathrm{H}(35 \mathrm{C})$ & 9315 & 4533 & 5845 & 353 \\
\hline$H(36 A)$ & 6472 & 3700 & 4047 & 583 \\
\hline $\mathrm{H}(36 \mathrm{~B})$ & 5348 & 3753 & 4653 & 583 \\
\hline $\mathrm{H}(36 \mathrm{C})$ & 6716 & 3470 & 5210 & 583 \\
\hline $\mathrm{H}(34 \mathrm{~A})$ & 6356 & 4625 & 3884 & 573 \\
\hline $\mathrm{H}(34 \mathrm{~B})$ & 6609 & 4908 & 4975 & 573 \\
\hline $\mathrm{H}(34 \mathrm{C})$ & 5263 & 4605 & 4528 & 573 \\
\hline
\end{tabular}

Table 6. Torsion angles $\left[^{\circ}\right]$ for 9,10-bis[bis(trimethylsilylethynyl)methylene]-9-10-dihydroanthracene (17).

\begin{tabular}{|c|c|}
\hline $\bar{C}(23)-\mathrm{C}(24)-\mathrm{Si}(4)-\mathrm{C}(36)$ & $-30.9(16)$ \\
\hline $\mathrm{C}(23)-\mathrm{C}(24)-\mathrm{Si}(4)-\mathrm{C}(35)$ & $-162.7(15)$ \\
\hline $\mathrm{C}(23)-\mathrm{C}(24)-\mathrm{Si}(4)-\mathrm{C}(34)$ & $79.9(16)$ \\
\hline$C(7)-C(2)-C(3)-C(4)$ & $-0.3(3)$ \\
\hline $\mathrm{C}(1)-\mathrm{C}(2)-\mathrm{C}(3)-\mathrm{C}(4)$ & $-178.2(2)$ \\
\hline$C(3)-C(2)-C(7)-C(6)$ & $0.0(3)$ \\
\hline$C(1)-C(2)-C(7)-C(6)$ & $178.0(2)$ \\
\hline $\mathrm{C}(3)-\mathrm{C}(2)-\mathrm{C}(7)-\mathrm{C}(8)$ & $-177.3(2)$ \\
\hline $\mathrm{C}(1)-\mathrm{C}(2)-\mathrm{C}(7)-\mathrm{C}(8)$ & $0.7(3)$ \\
\hline$C(3)-C(2)-C(1)-C(15)$ & $-36.2(3)$ \\
\hline $\mathrm{C}(7)-\mathrm{C}(2)-\mathrm{C}(1)-\mathrm{C}(15)$ & $145.9(2)$ \\
\hline $\mathrm{C}(3)-\mathrm{C}(2)-\mathrm{C}(1)-\mathrm{C}(14)$ & $144.2(2)$ \\
\hline $\mathrm{C}(7)-\mathrm{C}(2)-\mathrm{C}(1)-\mathrm{C}(14)$ & $-33.8(3)$ \\
\hline $\mathrm{C}(6)-\mathrm{C}(7)-\mathrm{C}(8)-\mathrm{C}(20)$ & $37.3(3)$ \\
\hline $\mathrm{C}(2)-\mathrm{C}(7)-\mathrm{C}(8)-\mathrm{C}(20)$ & $-145.5(2)$ \\
\hline $\mathrm{C}(6)-\mathrm{C}(7)-\mathrm{C}(8)-\mathrm{C}(9)$ & $-143.9(2)$ \\
\hline $\mathrm{C}(2)-\mathrm{C}(7)-\mathrm{C}(8)-\mathrm{C}(9)$ & $33.3(3)$ \\
\hline $\mathrm{C}(10)-\mathrm{C}(9)-\mathrm{C}(8)-\mathrm{C}(20)$ & $-40.6(3)$ \\
\hline$C(14)-C(9)-C(8)-C(20)$ & $144.5(2)$ \\
\hline $\mathrm{C}(10)-\mathrm{C}(9)-\mathrm{C}(8)-\mathrm{C}(7)$ & $140.6(2)$ \\
\hline $\mathrm{C}(14)-\mathrm{C}(9)-\mathrm{C}(8)-\mathrm{C}(7)$ & $-34.3(3)$ \\
\hline $\mathrm{C}(26)-\mathrm{Si}(1)-\mathrm{C}(19)-\mathrm{C}(18)$ & $130.6(18)$ \\
\hline $\mathrm{C}(27)-\mathrm{Si}(1)-\mathrm{C}(19)-\mathrm{C}(18)$ & $-108.7(18)$ \\
\hline $\mathrm{C}(25)-\mathrm{Si}(1)-\mathrm{C}(19)-\mathrm{C}(18)$ & $10.4(19)$ \\
\hline $\mathrm{C}(14)-\mathrm{C}(1)-\mathrm{C}(15)-\mathrm{C}(18)$ & $8.0(4)$ \\
\hline $\mathrm{C}(2)-\mathrm{C}(1)-\mathrm{C}(15)-\mathrm{C}(18)$ & $-171.6(2)$ \\
\hline $\mathrm{C}(14)-\mathrm{C}(1)-\mathrm{C}(15)-\mathrm{C}(16)$ & $-172.7(2)$ \\
\hline
\end{tabular}




\begin{tabular}{|c|c|}
\hline$C(2)-C(1)-C(15)-C(16)$ & $7.7(4)$ \\
\hline$C(7)-C(8)-C(20)-C(23)$ & $176.9(2)$ \\
\hline $\mathrm{C}(9)-\mathrm{C}(8)-\mathrm{C}(20)-\mathrm{C}(23)$ & $-1.8(4)$ \\
\hline$C(7)-C(8)-C(20)-C(21)$ & $0.0(4)$ \\
\hline $\mathrm{C}(9)-\mathrm{C}(8)-\mathrm{C}(20)-\mathrm{C}(21)$ & $-178.7(2)$ \\
\hline $\mathrm{C}(10)-\mathrm{C}(9)-\mathrm{C}(14)-\mathrm{C}(13)$ & $1.9(4)$ \\
\hline $\mathrm{C}(8)-\mathrm{C}(9)-\mathrm{C}(14)-\mathrm{C}(13)$ & $176.9(2)$ \\
\hline$C(10)-C(9)-C(14)-C(1)$ & $-173.8(2)$ \\
\hline $\mathrm{C}(8)-\mathrm{C}(9)-\mathrm{C}(14)-\mathrm{C}(1)$ & $1.2(3)$ \\
\hline $\mathrm{C}(15)-\mathrm{C}(1)-\mathrm{C}(14)-\mathrm{C}(13)$ & $37.8(4)$ \\
\hline $\mathrm{C}(2)-\mathrm{C}(1)-\mathrm{C}(14)-\mathrm{C}(13)$ & $-142.6(2)$ \\
\hline$C(15)-C(1)-C(14)-C(9)$ & $-146.7(2)$ \\
\hline $\mathrm{C}(2)-\mathrm{C}(1)-\mathrm{C}(14)-\mathrm{C}(9)$ & $33.0(3)$ \\
\hline $\mathrm{Si}(1)-\mathrm{C}(19)-\mathrm{C}(18)-\mathrm{C}(15)$ & $165(5)$ \\
\hline $\mathrm{C}(1)-\mathrm{C}(15)-\mathrm{C}(18)-\mathrm{C}(19)$ & $155(6)$ \\
\hline $\mathrm{C}(16)-\mathrm{C}(15)-\mathrm{C}(18)-\mathrm{C}(19)$ & $-24(6)$ \\
\hline$C(4)-C(5)-C(6)-C(7)$ & $0.1(4)$ \\
\hline$C(2)-C(7)-C(6)-C(5)$ & $0.1(3)$ \\
\hline $\mathrm{C}(8)-\mathrm{C}(7)-\mathrm{C}(6)-\mathrm{C}(5)$ & $177.3(2)$ \\
\hline $\mathrm{C}(8)-\mathrm{C}(20)-\mathrm{C}(21)-\mathrm{C}(22)$ & $-145(4)$ \\
\hline $\mathrm{C}(23)-\mathrm{C}(20)-\mathrm{C}(21)-\mathrm{C}(22)$ & $38(4)$ \\
\hline $\mathrm{C}(9)-\mathrm{C}(14)-\mathrm{C}(13)-\mathrm{C}(12)$ & $-0.7(4)$ \\
\hline $\mathrm{C}(1)-\mathrm{C}(14)-\mathrm{C}(13)-\mathrm{C}(12)$ & $174.9(2)$ \\
\hline$C(2)-C(3)-C(4)-C(5)$ & $0.4(4)$ \\
\hline$C(6)-C(5)-C(4)-C(3)$ & $-0.3(4)$ \\
\hline $\mathrm{Si}(4)-\mathrm{C}(24)-\mathrm{C}(23)-\mathrm{C}(20)$ & $-148(10)$ \\
\hline $\mathrm{C}(8)-\mathrm{C}(20)-\mathrm{C}(23)-\mathrm{C}(24)$ & $158(11)$ \\
\hline $\mathrm{C}(21)-\mathrm{C}(20)-\mathrm{C}(23)-\mathrm{C}(24)$ & $-25(11)$ \\
\hline $\mathrm{C}(20)-\mathrm{C}(21)-\mathrm{C}(22)-\mathrm{Si}(2)$ & $140(3)$ \\
\hline $\mathrm{C}(32)-\mathrm{Si}(2)-\mathrm{C}(22)-\mathrm{C}(21)$ & $-129.4(18)$ \\
\hline $\mathrm{C}(33)-\mathrm{Si}(2)-\mathrm{C}(22)-\mathrm{C}(21)$ & $110.0(19)$ \\
\hline $\mathrm{C}(31)-\mathrm{Si}(2)-\mathrm{C}(22)-\mathrm{C}(21)$ & $-9.7(19)$ \\
\hline $\mathrm{C}(1)-\mathrm{C}(15)-\mathrm{C}(16)-\mathrm{C}(17)$ & $140(3)$ \\
\hline $\mathrm{C}(18)-\mathrm{C}(15)-\mathrm{C}(16)-\mathrm{C}(17)$ & $-40(3)$ \\
\hline$C(15)-C(16)-C(17)-S i(3)$ & $-91(3)$ \\
\hline $\mathrm{C}(29)-\mathrm{Si}(3)-\mathrm{C}(17)-\mathrm{C}(16)$ & $171.3(13)$ \\
\hline $\mathrm{C}(30)-\mathrm{Si}(3)-\mathrm{C}(17)-\mathrm{C}(16)$ & $-69.2(13)$ \\
\hline $\mathrm{C}(28)-\mathrm{Si}(3)-\mathrm{C}(17)-\mathrm{C}(16)$ & $50.5(13)$ \\
\hline $\mathrm{C}(14)-\mathrm{C}(9)-\mathrm{C}(10)-\mathrm{C}(11)$ & $-2.1(4)$ \\
\hline$C(8)-C(9)-C(10)-C(11)$ & $-176.9(2)$ \\
\hline $\mathrm{C}(14)-\mathrm{C}(13)-\mathrm{C}(12)-\mathrm{C}(11)$ & $-0.3(4)$ \\
\hline $\mathrm{C}(13)-\mathrm{C}(12)-\mathrm{C}(11)-\mathrm{C}(10)$ & $0.1(4)$ \\
\hline $\mathrm{C}(9)-\mathrm{C}(10)-\mathrm{C}(11)-\mathrm{C}(12)$ & $1.1(4)$ \\
\hline
\end{tabular}


X-ray data for 3,10-di-t-butylbenzo[c]phenanthrene (9b):
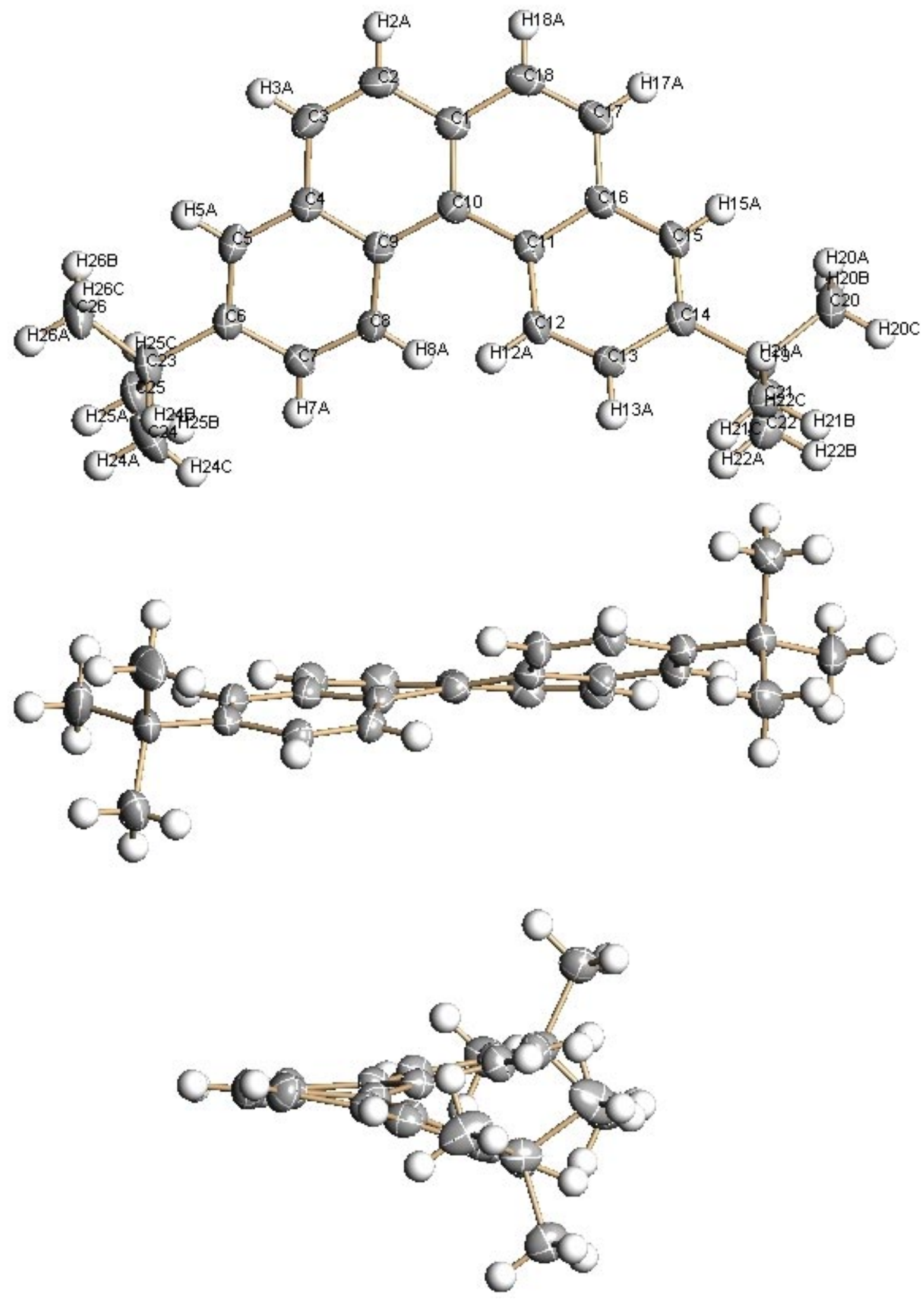

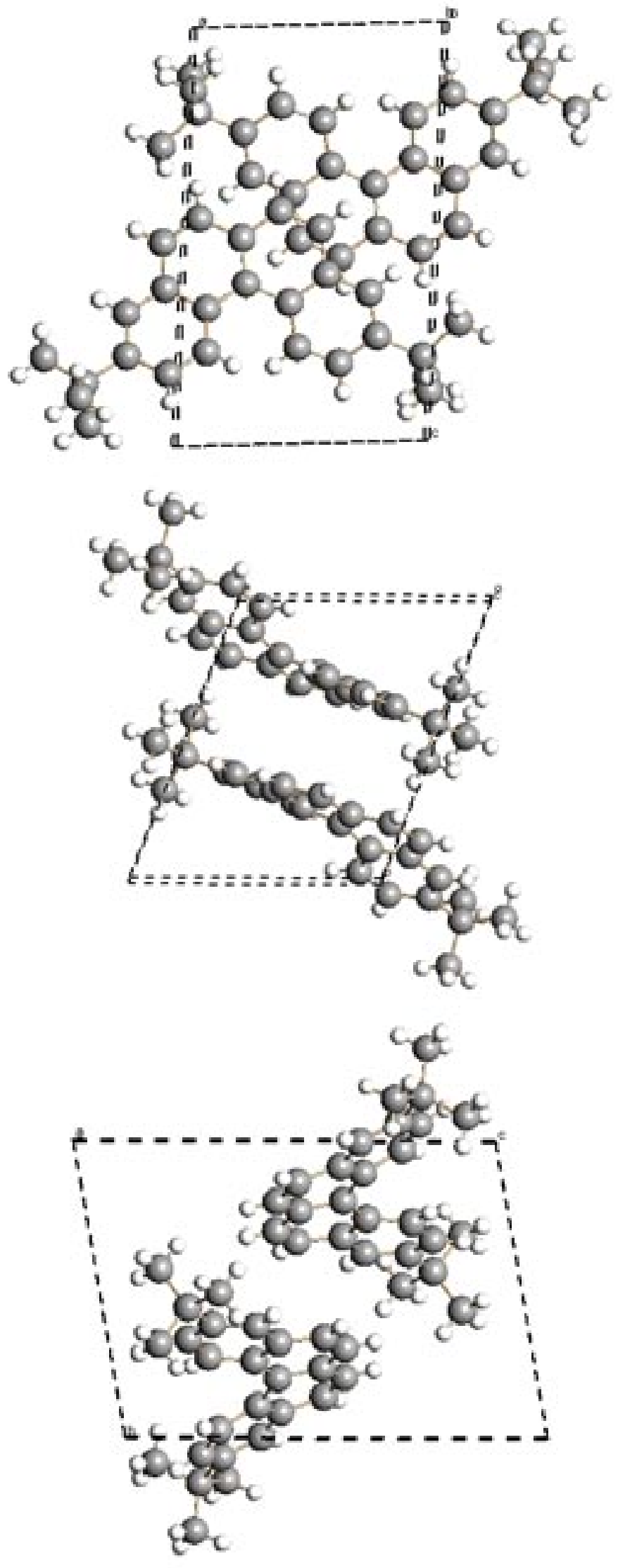

page S37 
Table 1. Crystal data and structure refinement for 3,10-di-t-butylbenzo[c]phenanthrene (9b).

Identification code

Empirical formula

Formula weight

Temperature

Wavelength

Crystal system

Space group

Unit cell dimensions

Volume

Z

Density (calculated)

Absorption coefficient

$\mathrm{F}(000)$

Crystal size

Theta range for data collection

Index ranges

Reflections collected

Independent reflections

Completeness to theta $=28.35^{\circ}$

Absorption correction

Refinement method

Data / restraints / parameters

Goodness-of-fit on $\mathrm{F}^{2}$

Final R indices [I $>2$ sigma(I)]

$\mathrm{R}$ indices (all data)

Largest diff. peak and hole pat $05 \mathrm{t}$

C26 H28

340.48

193(2) K

$0.71073 \AA$

Triclinic

P-1

$\mathrm{a}=8.2248(12) \AA$

$\alpha=77.512(4)^{\circ}$.

$\mathrm{b}=9.9887(15) \AA$

$\beta=81.672(4)^{\circ}$.

$\mathrm{c}=13.0969(18) \AA$

$\gamma=67.844(4)^{\circ}$.

$970.6(2) \AA^{3}$

2

$1.165 \mathrm{Mg} / \mathrm{m}^{3}$

$0.065 \mathrm{~mm}^{-1}$

368

$0.22 \times 0.20 \times 0.08 \mathrm{~mm}^{3}$

1.60 to $28.35^{\circ}$.

$-5<=\mathrm{h}<=10,-10<=\mathrm{k}<=13,-17<=1<=16$

7287

$4774[\mathrm{R}(\mathrm{int})=0.0335]$

$98.5 \%$

None

Full-matrix least-squares on $\mathrm{F}^{2}$

4774 / 0 / 235

0.844

$\mathrm{R} 1=0.0558, \mathrm{wR} 2=0.0956$

$\mathrm{R} 1=0.1143, \mathrm{wR} 2=0.1117$

0.229 and -0.143 e. $\AA^{-3}$

Table 2. Atomic coordinates ( $\left.\times 10^{4}\right)$ and equivalent isotropic displacement parameters $\left(\AA^{2} \times 10^{3}\right)$ for 3,10-di-t-butylbenzo[c]phenanthrene (9b). U(eq) is defined as one third of the trace of the orthogonalized $\mathrm{U}^{\mathrm{ij}}$ tensor.

\begin{tabular}{lrrrr}
\hline & $\mathrm{x}$ & $\mathrm{y}$ & $\mathrm{z}$ & $\mathrm{U}(\mathrm{eq})$ \\
\hline $\mathrm{C}(10)$ & & & & \\
$\mathrm{C}(6)$ & $2490(2)$ & $7984(2)$ & $3855(1)$ & $32(1)$ \\
$\mathrm{C}(14)$ & $-2177(2)$ & $10623(2)$ & $2208(1)$ & $33(1)$ \\
$\mathrm{C}(9)$ & $7819(2)$ & $6214(2)$ & $2523(1)$ & $33(1)$ \\
$\mathrm{C}(1)$ & $954(2)$ & $8897(2)$ & $3276(1)$ & $30(1)$ \\
$\mathrm{C}(4)$ & $2239(2)$ & $7632(2)$ & $4949(1)$ & $36(1)$ \\
$\mathrm{C}(7)$ & $-744(2)$ & $9106(2)$ & $3787(1)$ & $34(1)$ \\
$\mathrm{C}(19)$ & $-496(2)$ & $10541(2)$ & $1748(1)$ & $34(1)$ \\
$\mathrm{C}(5)$ & $9687(2)$ & $5658(2)$ & $2016(1)$ & $36(1)$ \\
$\mathrm{C}(11)$ & $-2257(2)$ & $9918(2)$ & $3226(1)$ & $37(1)$ \\
$\mathrm{C}(16)$ & $4262(2)$ & $7399(2)$ & $3376(1)$ & $30(1)$ \\
$\mathrm{C}(8)$ & $5701(2)$ & $6793(2)$ & $4021(1)$ & $33(1)$ \\
$\mathrm{C}(13)$ & $1003(2)$ & $9709(2)$ & $2254(1)$ & $32(1)$ \\
$\mathrm{C}(15)$ & $6360(2)$ & $6698(2)$ & $1905(1)$ & $36(1)$ \\
$\mathrm{C}(23)$ & $7435(2)$ & $6254(2)$ & $3570(1)$ & $37(1)$ \\
$\mathrm{C}(12)$ & $-3808(2)$ & $11459(2)$ & $1581(2)$ & $39(1)$ \\
$\mathrm{C}(2)$ & $4663(2)$ & $7269(2)$ & $2309(1)$ & $34(1)$ \\
$\mathrm{C}(18)$ & $512(2)$ & $7932(2)$ & $5440(1)$ & $42(1)$ \\
$\mathrm{C}(17)$ & $3717(2)$ & $6968(2)$ & $5570(1)$ & $42(1)$ \\
$\mathrm{C}(3)$ & $5372(2)$ & $6626(2)$ & $5130(1)$ & $42(1)$ \\
$\mathrm{C}(20)$ & $-918(2)$ & $8568(2)$ & $4880(1)$ & $42(1)$ \\
& $11072(2)$ & $5177(2)$ & $2813(2)$ & $48(1)$
\end{tabular}




\begin{tabular}{|c|c|c|c|c|}
\hline $\mathrm{C}(21)$ & $9927(2)$ & $4352(2)$ & 1499(1) & $42(1)$ \\
\hline$C(26)$ & $-5473(2)$ & $11358(2)$ & $2220(2)$ & $59(1)$ \\
\hline $\mathrm{C}(22)$ & $9976(2)$ & $6886(2)$ & $1180(2)$ & $48(1)$ \\
\hline $\mathrm{C}(25)$ & $-4020(2)$ & $13077(2)$ & $1252(2)$ & $48(1)$ \\
\hline $\mathrm{C}(24)$ & $-3573(3)$ & $10783(2)$ & $601(2)$ & $62(1)$ \\
\hline
\end{tabular}

Table 3. Bond lengths $[\AA]$ and angles $\left[^{\circ}\right]$ for 3,10-di-t-butylbenzo $[c]$ phenanthrene (9b).

\begin{tabular}{|c|c|c|c|}
\hline $\mathrm{C}(10)-\mathrm{C}(1)$ & $1.404(2)$ & $C(15)-C(14)-C(19)$ & $123.89(15)$ \\
\hline $\mathrm{C}(10)-\mathrm{C}(11)$ & $1.453(2)$ & $\mathrm{C}(13)-\mathrm{C}(14)-\mathrm{C}(19)$ & $120.01(15)$ \\
\hline $\mathrm{C}(10)-\mathrm{C}(9)$ & $1.453(2)$ & $\mathrm{C}(8)-\mathrm{C}(9)-\mathrm{C}(4)$ & $115.93(14)$ \\
\hline$C(6)-C(5)$ & $1.372(2)$ & $\mathrm{C}(8)-\mathrm{C}(9)-\mathrm{C}(10)$ & $124.92(15)$ \\
\hline$C(6)-C(7)$ & $1.406(2)$ & $\mathrm{C}(4)-\mathrm{C}(9)-\mathrm{C}(10)$ & $118.93(16)$ \\
\hline $\mathrm{C}(6)-\mathrm{C}(23)$ & $1.533(2)$ & $C(10)-C(1)-C(2)$ & $120.24(16)$ \\
\hline$C(14)-C(15)$ & $1.367(2)$ & $\mathrm{C}(10)-\mathrm{C}(1)-\mathrm{C}(18)$ & $120.02(16)$ \\
\hline$C(14)-C(13)$ & $1.415(2)$ & $\mathrm{C}(2)-\mathrm{C}(1)-\mathrm{C}(18)$ & $119.74(17)$ \\
\hline $\mathrm{C}(14)-\mathrm{C}(19)$ & $1.527(2)$ & $\mathrm{C}(5)-\mathrm{C}(4)-\mathrm{C}(9)$ & $120.09(16)$ \\
\hline $\mathrm{C}(9)-\mathrm{C}(8)$ & $1.412(2)$ & $C(5)-C(4)-C(3)$ & $120.07(16)$ \\
\hline $\mathrm{C}(9)-\mathrm{C}(4)$ & $1.418(2)$ & $\mathrm{C}(9)-\mathrm{C}(4)-\mathrm{C}(3)$ & $119.74(15)$ \\
\hline$C(1)-C(2)$ & $1.421(2)$ & $\mathrm{C}(8)-\mathrm{C}(7)-\mathrm{C}(6)$ & $122.14(17)$ \\
\hline $\mathrm{C}(1)-\mathrm{C}(18)$ & $1.422(2)$ & $\mathrm{C}(22)-\mathrm{C}(19)-\mathrm{C}(14)$ & $109.07(14)$ \\
\hline $\mathrm{C}(4)-\mathrm{C}(5)$ & $1.411(2)$ & $\mathrm{C}(22)-\mathrm{C}(19)-\mathrm{C}(20)$ & $108.33(16)$ \\
\hline $\mathrm{C}(4)-\mathrm{C}(3)$ & $1.422(2)$ & $\mathrm{C}(14)-\mathrm{C}(19)-\mathrm{C}(20)$ & $111.95(15)$ \\
\hline $\mathrm{C}(7)-\mathrm{C}(8)$ & $1.369(2)$ & $\mathrm{C}(22)-\mathrm{C}(19)-\mathrm{C}(21)$ & $109.08(15)$ \\
\hline $\mathrm{C}(19)-\mathrm{C}(22)$ & $1.524(2)$ & $\mathrm{C}(14)-\mathrm{C}(19)-\mathrm{C}(21)$ & $109.45(15)$ \\
\hline$C(19)-C(20)$ & $1.528(2)$ & $\mathrm{C}(20)-\mathrm{C}(19)-\mathrm{C}(21)$ & $108.92(14)$ \\
\hline $\mathrm{C}(19)-\mathrm{C}(21)$ & $1.532(2)$ & $C(6)-C(5)-C(4)$ & $122.67(16)$ \\
\hline $\mathrm{C}(11)-\mathrm{C}(12)$ & $1.411(2)$ & $C(12)-C(11)-C(16)$ & $116.28(15)$ \\
\hline $\mathrm{C}(11)-\mathrm{C}(16)$ & $1.417(2)$ & $\mathrm{C}(12)-\mathrm{C}(11)-\mathrm{C}(10)$ & $124.38(15)$ \\
\hline$C(16)-C(15)$ & $1.410(2)$ & $\mathrm{C}(16)-\mathrm{C}(11)-\mathrm{C}(10)$ & $119.11(15)$ \\
\hline$C(16)-C(17)$ & $1.423(2)$ & $C(15)-C(16)-C(11)$ & $120.05(15)$ \\
\hline $\mathrm{C}(13)-\mathrm{C}(12)$ & $1.367(2)$ & $\mathrm{C}(15)-\mathrm{C}(16)-\mathrm{C}(17)$ & $120.35(15)$ \\
\hline$C(23)-C(26)$ & $1.525(3)$ & $\mathrm{C}(11)-\mathrm{C}(16)-\mathrm{C}(17)$ & $119.41(15)$ \\
\hline$C(23)-C(25)$ & $1.528(2)$ & $\mathrm{C}(7)-\mathrm{C}(8)-\mathrm{C}(9)$ & $122.02(16)$ \\
\hline$C(23)-C(24)$ & $1.531(2)$ & $\mathrm{C}(12)-\mathrm{C}(13)-\mathrm{C}(14)$ & $122.46(16)$ \\
\hline $\mathrm{C}(2)-\mathrm{C}(3)$ & $1.346(2)$ & $C(14)-C(15)-C(16)$ & $123.05(16)$ \\
\hline \multirow[t]{4}{*}{$\mathrm{C}(18)-\mathrm{C}(17)$} & $1.345(2)$ & $\mathrm{C}(26)-\mathrm{C}(23)-\mathrm{C}(25)$ & $108.71(14)$ \\
\hline & & $C(26)-C(23)-C(24)$ & $108.59(17)$ \\
\hline & & $\mathrm{C}(25)-\mathrm{C}(23)-\mathrm{C}(24)$ & $109.37(16)$ \\
\hline & & $C(26)-C(23)-C(6)$ & $111.43(16)$ \\
\hline$C(1)-C(10)-C(11)$ & $117.88(15)$ & $C(25)-C(23)-C(6)$ & $110.13(15)$ \\
\hline $\mathrm{C}(1)-\mathrm{C}(10)-\mathrm{C}(9)$ & $117.71(15)$ & $\mathrm{C}(24)-\mathrm{C}(23)-\mathrm{C}(6)$ & $108.58(14)$ \\
\hline$C(11)-C(10)-C(9)$ & $124.40(16)$ & $\mathrm{C}(13)-\mathrm{C}(12)-\mathrm{C}(11)$ & $121.66(15)$ \\
\hline$C(5)-C(6)-C(7)$ & $116.53(15)$ & $\mathrm{C}(3)-\mathrm{C}(2)-\mathrm{C}(1)$ & $121.48(17)$ \\
\hline$C(5)-C(6)-C(23)$ & $123.00(16)$ & $\mathrm{C}(17)-\mathrm{C}(18)-\mathrm{C}(1)$ & $121.41(17)$ \\
\hline$C(7)-C(6)-C(23)$ & $120.47(16)$ & $\mathrm{C}(18)-\mathrm{C}(17)-\mathrm{C}(16)$ & $120.64(16)$ \\
\hline $\mathrm{C}(15)-\mathrm{C}(14)-\mathrm{C}(13)$ & $116.09(15)$ & $C(2)-C(3)-C(4)$ & $120.29(17)$ \\
\hline
\end{tabular}


Table 4. Anisotropic displacement parameters $\left(\AA^{2} \times 10^{3}\right)$ for 3,10-di-t-butylbenzo[c]phenanthrene (9b). The anisotropic displacement factor exponent takes the form: $-2 \pi^{2}\left[h^{2} a^{* 2} U^{11}+\ldots+2 h k a^{*} b^{*} U^{12}\right]$

\begin{tabular}{lcccccc}
\hline & $\mathrm{U}^{11}$ & $\mathrm{U}^{22}$ & $\mathrm{U}^{33}$ & $\mathrm{U}^{23}$ & $\mathrm{U}^{13}$ & $\mathrm{U}^{12}$ \\
\hline $\mathrm{C}(10)$ & $34(1)$ & $31(1)$ & $32(1)$ & $-7(1)$ & $-6(1)$ & $-12(1)$ \\
$\mathrm{C}(6)$ & $28(1)$ & $31(1)$ & $41(1)$ & $-10(1)$ & $-4(1)$ & $-8(1)$ \\
$\mathrm{C}(14)$ & $31(1)$ & $30(1)$ & $38(1)$ & $-5(1)$ & $-7(1)$ & $-9(1)$ \\
$\mathrm{C}(9)$ & $30(1)$ & $30(1)$ & $32(1)$ & $-10(1)$ & $-3(1)$ & $-11(1)$ \\
$\mathrm{C}(1)$ & $38(1)$ & $33(1)$ & $35(1)$ & $-7(1)$ & $-4(1)$ & $-10(1)$ \\
$\mathrm{C}(4)$ & $31(1)$ & $34(1)$ & $37(1)$ & $-8(1)$ & $-1(1)$ & $-12(1)$ \\
$\mathrm{C}(7)$ & $32(1)$ & $36(1)$ & $32(1)$ & $-5(1)$ & $-6(1)$ & $-10(1)$ \\
$\mathrm{C}(19)$ & $29(1)$ & $33(1)$ & $43(1)$ & $-5(1)$ & $-6(1)$ & $-9(1)$ \\
$\mathrm{C}(5)$ & $26(1)$ & $38(1)$ & $48(1)$ & $-13(1)$ & $2(1)$ & $-12(1)$ \\
$\mathrm{C}(11)$ & $31(1)$ & $28(1)$ & $33(1)$ & $-5(1)$ & $-7(1)$ & $-9(1)$ \\
$\mathrm{C}(16)$ & $32(1)$ & $35(1)$ & $33(1)$ & $-8(1)$ & $-9(1)$ & $-10(1)$ \\
$\mathrm{C}(8)$ & $27(1)$ & $38(1)$ & $33(1)$ & $-9(1)$ & $-1(1)$ & $-12(1)$ \\
$\mathrm{C}(13)$ & $35(1)$ & $40(1)$ & $31(1)$ & $-7(1)$ & $-5(1)$ & $-10(1)$ \\
$\mathrm{C}(15)$ & $31(1)$ & $38(1)$ & $40(1)$ & $-7(1)$ & $-13(1)$ & $-7(1)$ \\
$\mathrm{C}(23)$ & $26(1)$ & $34(1)$ & $55(1)$ & $-6(1)$ & $-12(1)$ & $-6(1)$ \\
$\mathrm{C}(12)$ & $27(1)$ & $39(1)$ & $34(1)$ & $-7(1)$ & $-9(1)$ & $-7(1)$ \\
$\mathrm{C}(2)$ & $48(1)$ & $46(1)$ & $30(1)$ & $-3(1)$ & $0(1)$ & $-17(1)$ \\
$\mathrm{C}(18)$ & $50(1)$ & $44(1)$ & $30(1)$ & $-7(1)$ & $-7(1)$ & $-11(1)$ \\
$\mathrm{C}(17)$ & $42(1)$ & $48(1)$ & $33(1)$ & $-7(1)$ & $-13(1)$ & $-9(1)$ \\
$\mathrm{C}(3)$ & $38(1)$ & $45(1)$ & $40(1)$ & $-6(1)$ & $6(1)$ & $-15(1)$ \\
$\mathrm{C}(20)$ & $30(1)$ & $51(1)$ & $62(1)$ & $-19(1)$ & $-7(1)$ & $-9(1)$ \\
$\mathrm{C}(21)$ & $39(1)$ & $38(1)$ & $47(1)$ & $-9(1)$ & $-3(1)$ & $-9(1)$ \\
$\mathrm{C}(26)$ & $32(1)$ & $51(1)$ & $89(2)$ & $6(1)$ & $-17(1)$ & $-13(1)$ \\
$\mathrm{C}(22)$ & $40(1)$ & $43(1)$ & $59(1)$ & $-9(1)$ & $4(1)$ & $-14(1)$ \\
$\mathrm{C}(25)$ & $36(1)$ & $42(1)$ & $59(1)$ & $-1(1)$ & $-12(1)$ & $-7(1)$ \\
$\mathrm{C}(24)$ & $53(1)$ & $59(2)$ & $74(2)$ & $-21(1)$ & $-38(1)$ & $-2(1)$ \\
& & & & & & \\
\hline & & & & & &
\end{tabular}

Table 5. Hydrogen coordinates ( $\left.\mathrm{x} 10^{4}\right)$ and isotropic displacement parameters $\left(\AA^{2} \mathrm{x} 10^{3}\right)$ for 3,10-di-t-butylbenzo[c]phenanthrene (9b).

\begin{tabular}{lrrrl}
\hline & $\mathrm{x}$ & $\mathrm{y}$ & $\mathrm{z}$ & $\mathrm{U}(\mathrm{eq})$ \\
\hline $\mathrm{H}(7 \mathrm{~A})$ & & & & \\
$\mathrm{H}(5 \mathrm{~A})$ & -398 & 11069 & 1079 & 40 \\
$\mathrm{H}(8 \mathrm{~A})$ & -3354 & 9978 & 3561 & 44 \\
$\mathrm{H}(13 \mathrm{~A})$ & 2087 & 9678 & 1913 & 38 \\
$\mathrm{H}(15 \mathrm{~A})$ & 6563 & 6625 & 1199 & 43 \\
$\mathrm{H}(12 \mathrm{~A})$ & 8361 & 5911 & 4004 & 44 \\
$\mathrm{H}(2 \mathrm{~A})$ & 3749 & 7580 & 1869 & 41 \\
$\mathrm{H}(18 \mathrm{~A})$ & 367 & 7683 & 6165 & 51 \\
$\mathrm{H}(17 \mathrm{~A})$ & 3534 & 6766 & 6295 & 51 \\
$\mathrm{H}(3 \mathrm{~A})$ & 6311 & 6278 & 5555 & 50 \\
$\mathrm{H}(20 \mathrm{~A})$ & -2025 & 8656 & 5208 & 50 \\
$\mathrm{H}(20 \mathrm{~B})$ & 10915 & 4398 & 3344 & 71 \\
$\mathrm{H}(20 \mathrm{C})$ & 10945 & 5994 & 3132 & 71 \\
$\mathrm{H}(21 \mathrm{~A})$ & 12225 & 4838 & 2465 & 71 \\
$\mathrm{H}(21 \mathrm{~B})$ & 9750 & 3574 & 2022 & 63 \\
$\mathrm{H}(21 \mathrm{C})$ & 11095 & 4013 & 1170 & 63 \\
$\mathrm{H}(26 \mathrm{~A})$ & 9084 & 4653 & 981 & 63 \\
$\mathrm{H}(26 \mathrm{~B})$ & -6468 & 11886 & 1806 & 89 \\
& -5635 & 11776 & 2838 & 89
\end{tabular}




\begin{tabular}{|c|c|c|c|c|}
\hline $\mathrm{H}(26 \mathrm{C})$ & -5365 & 10346 & 2419 & 89 \\
\hline $\mathrm{H}(22 \mathrm{~A})$ & 9120 & 7198 & 669 & 72 \\
\hline $\mathrm{H}(22 \mathrm{~B})$ & 11137 & 6534 & 843 & 72 \\
\hline $\mathrm{H}(22 \mathrm{C})$ & 9852 & 7700 & 1502 & 72 \\
\hline $\mathrm{H}(25 \mathrm{~A})$ & -5040 & 13590 & 856 & 72 \\
\hline $\mathrm{H}(25 \mathrm{~B})$ & -2995 & 13157 & 829 & 72 \\
\hline $\mathrm{H}(25 \mathrm{C})$ & -4160 & 13501 & 1866 & 72 \\
\hline $\mathrm{H}(24 \mathrm{~A})$ & -4586 & 11299 & 200 & 93 \\
\hline $\mathrm{H}(24 \mathrm{~B})$ & -3446 & 9768 & 806 & 93 \\
\hline $\mathrm{H}(24 \mathrm{C})$ & -2542 & 10859 & 183 & 93 \\
\hline
\end{tabular}

Table 6. Torsion angles $\left[{ }^{\circ}\right]$ for 3,10-di-t-butylbenzo[c]phenanthrene (9b).

\begin{tabular}{|c|c|}
\hline $\bar{C}(1)-C(10)-C(9)-C(8)$ & $160.02(18)$ \\
\hline $\mathrm{C}(11)-\mathrm{C}(10)-\mathrm{C}(9)-\mathrm{C}(8)$ & $-20.7(3)$ \\
\hline $\mathrm{C}(1)-\mathrm{C}(10)-\mathrm{C}(9)-\mathrm{C}(4)$ & $-14.4(2)$ \\
\hline $\mathrm{C}(11)-\mathrm{C}(10)-\mathrm{C}(9)-\mathrm{C}(4)$ & $164.85(17)$ \\
\hline $\mathrm{C}(11)-\mathrm{C}(10)-\mathrm{C}(1)-\mathrm{C}(2)$ & $-167.99(17)$ \\
\hline $\mathrm{C}(9)-\mathrm{C}(10)-\mathrm{C}(1)-\mathrm{C}(2)$ & $11.3(3)$ \\
\hline $\mathrm{C}(11)-\mathrm{C}(10)-\mathrm{C}(1)-\mathrm{C}(18)$ & $11.8(3)$ \\
\hline $\mathrm{C}(9)-\mathrm{C}(10)-\mathrm{C}(1)-\mathrm{C}(18)$ & $-168.95(17)$ \\
\hline $\mathrm{C}(8)-\mathrm{C}(9)-\mathrm{C}(4)-\mathrm{C}(5)$ & $8.7(2)$ \\
\hline $\mathrm{C}(10)-\mathrm{C}(9)-\mathrm{C}(4)-\mathrm{C}(5)$ & $-176.44(17)$ \\
\hline$C(8)-C(9)-C(4)-C(3)$ & $-167.69(17)$ \\
\hline$C(10)-C(9)-C(4)-C(3)$ & $7.2(3)$ \\
\hline$C(5)-C(6)-C(7)-C(8)$ & $5.1(3)$ \\
\hline $\mathrm{C}(23)-\mathrm{C}(6)-\mathrm{C}(7)-\mathrm{C}(8)$ & $-174.73(17)$ \\
\hline $\mathrm{C}(15)-\mathrm{C}(14)-\mathrm{C}(19)-\mathrm{C}(22)$ & $-120.8(2)$ \\
\hline $\mathrm{C}(13)-\mathrm{C}(14)-\mathrm{C}(19)-\mathrm{C}(22)$ & $60.6(2)$ \\
\hline $\mathrm{C}(15)-\mathrm{C}(14)-\mathrm{C}(19)-\mathrm{C}(20)$ & $-0.9(3)$ \\
\hline $\mathrm{C}(13)-\mathrm{C}(14)-\mathrm{C}(19)-\mathrm{C}(20)$ & $-179.57(17)$ \\
\hline $\mathrm{C}(15)-\mathrm{C}(14)-\mathrm{C}(19)-\mathrm{C}(21)$ & 119.93(19) \\
\hline $\mathrm{C}(13)-\mathrm{C}(14)-\mathrm{C}(19)-\mathrm{C}(21)$ & $-58.7(2)$ \\
\hline$C(7)-C(6)-C(5)-C(4)$ & $-2.1(3)$ \\
\hline$C(23)-C(6)-C(5)-C(4)$ & $177.69(17)$ \\
\hline$C(9)-C(4)-C(5)-C(6)$ & $-4.9(3)$ \\
\hline $\mathrm{C}(3)-\mathrm{C}(4)-\mathrm{C}(5)-\mathrm{C}(6)$ & $171.45(18)$ \\
\hline $\mathrm{C}(1)-\mathrm{C}(10)-\mathrm{C}(11)-\mathrm{C}(12)$ & $160.16(18)$ \\
\hline $\mathrm{C}(9)-\mathrm{C}(10)-\mathrm{C}(11)-\mathrm{C}(12)$ & $-19.1(3)$ \\
\hline$C(1)-C(10)-C(11)-C(16)$ & $-14.1(2)$ \\
\hline$C(9)-C(10)-C(11)-C(16)$ & $166.70(17)$ \\
\hline$C(12)-C(11)-C(16)-C(15)$ & $7.0(3)$ \\
\hline $\mathrm{C}(10)-\mathrm{C}(11)-\mathrm{C}(16)-\mathrm{C}(15)$ & $-178.29(17)$ \\
\hline $\mathrm{C}(12)-\mathrm{C}(11)-\mathrm{C}(16)-\mathrm{C}(17)$ & $-168.01(17)$ \\
\hline $\mathrm{C}(10)-\mathrm{C}(11)-\mathrm{C}(16)-\mathrm{C}(17)$ & $6.7(3)$ \\
\hline$C(6)-C(7)-C(8)-C(9)$ & $-1.0(3)$ \\
\hline $\mathrm{C}(4)-\mathrm{C}(9)-\mathrm{C}(8)-\mathrm{C}(7)$ & $-5.9(3)$ \\
\hline $\mathrm{C}(10)-\mathrm{C}(9)-\mathrm{C}(8)-\mathrm{C}(7)$ & $179.53(16)$ \\
\hline $\mathrm{C}(15)-\mathrm{C}(14)-\mathrm{C}(13)-\mathrm{C}(12)$ & $4.3(3)$ \\
\hline $\mathrm{C}(19)-\mathrm{C}(14)-\mathrm{C}(13)-\mathrm{C}(12)$ & $-176.93(18)$ \\
\hline $\mathrm{C}(13)-\mathrm{C}(14)-\mathrm{C}(15)-\mathrm{C}(16)$ & $-2.3(3)$ \\
\hline$C(19)-C(14)-C(15)-C(16)$ & $179.02(17)$ \\
\hline $\mathrm{C}(11)-\mathrm{C}(16)-\mathrm{C}(15)-\mathrm{C}(14)$ & $-3.4(3)$ \\
\hline $\mathrm{C}(17)-\mathrm{C}(16)-\mathrm{C}(15)-\mathrm{C}(14)$ & $171.55(19)$ \\
\hline $\mathrm{C}(5)-\mathrm{C}(6)-\mathrm{C}(23)-\mathrm{C}(26)$ & $-1.8(3)$ \\
\hline $\mathrm{C}(7)-\mathrm{C}(6)-\mathrm{C}(23)-\mathrm{C}(26)$ & 177.98(17) \\
\hline
\end{tabular}


$\mathrm{C}(5)-\mathrm{C}(6)-\mathrm{C}(23)-\mathrm{C}(25)$

$\mathrm{C}(7)-\mathrm{C}(6)-\mathrm{C}(23)-\mathrm{C}(25)$

$\mathrm{C}(5)-\mathrm{C}(6)-\mathrm{C}(23)-\mathrm{C}(24)$

$\mathrm{C}(7)-\mathrm{C}(6)-\mathrm{C}(23)-\mathrm{C}(24)$

$\mathrm{C}(14)-\mathrm{C}(13)-\mathrm{C}(12)-\mathrm{C}(11)$

C(16)-C(11)-C(12)-C(13)

$\mathrm{C}(10)-\mathrm{C}(11)-\mathrm{C}(12)-\mathrm{C}(13)$

$\mathrm{C}(10)-\mathrm{C}(1)-\mathrm{C}(2)-\mathrm{C}(3)$

$\mathrm{C}(18)-\mathrm{C}(1)-\mathrm{C}(2)-\mathrm{C}(3)$

$\mathrm{C}(10)-\mathrm{C}(1)-\mathrm{C}(18)-\mathrm{C}(17)$

$\mathrm{C}(2)-\mathrm{C}(1)-\mathrm{C}(18)-\mathrm{C}(17)$

$\mathrm{C}(1)-\mathrm{C}(18)-\mathrm{C}(17)-\mathrm{C}(16)$

$\mathrm{C}(15)-\mathrm{C}(16)-\mathrm{C}(17)-\mathrm{C}(18)$

$\mathrm{C}(11)-\mathrm{C}(16)-\mathrm{C}(17)-\mathrm{C}(18)$

$\mathrm{C}(1)-\mathrm{C}(2)-\mathrm{C}(3)-\mathrm{C}(4)$

$\mathrm{C}(5)-\mathrm{C}(4)-\mathrm{C}(3)-\mathrm{C}(2)$

$\mathrm{C}(9)-\mathrm{C}(4)-\mathrm{C}(3)-\mathrm{C}(2)$
118.92(19)

$-61.3(2)$

$-121.4(2)$

$58.4(2)$

$-0.5(3)$

$-5.2(3)$

$-179.53(17)$

$-0.7(3)$

179.54(19)

$-1.9(3)$

177.88(19)

$-6.0(3)$

$-171.62(18)$

$3.4(3)$

$-7.0(3)$

$-172.82(18)$

$3.5(3)$ 